\title{
Processing of Garden Path Jokes: Theoretical Concepts and Empirical Correlates
}

\author{
Dissertation \\ zur Erlangung des mathematisch-naturwissenschaftlichen Doktorgrades \\ "Doctor rerum naturalium" \\ der Georg-August-Universität Göttingen \\ im Grundprogramm Biologie \\ der Georg-August University School of Science (GAUSS) \\ vorgelegt von \\ Bastian Mayerhofer \\ aus Hallein, Österreich
}

Göttingen, 2014 
Betreuungsausschuss:

- Prof. Dr. Annekathrin Schacht, Nachwuchsgruppe Experimentelle Psycholinguistik, Courant Forschungszentrum Textstrukturen, Universität Göttingen

- Prof. Dr. Hannes Rakoczy, Abteilung Biologische Entwicklungspsychologie, GeorgElias-Müller-Institut für Psychologie, Universität Göttingen

Mitglieder der Prüfungskommission:

- Referent: Prof. Dr. Annekathrin Schacht, Nachwuchsgruppe Experimentelle Psycholinguistik, Courant Forschungszentrum Textstrukturen, Universität Göttingen

- Koreferent: Prof. Dr. Hannes Rakoczy, Abteilung Biologische Entwicklungspsychologie, Georg-Elias-Müller-Institut für Psychologie, Universität Göttingen

Weitere Mitglieder der Prüfungskommission:

- Prof. Dr. Andreas Glöckner, Abteilung Psychologische Diagnostik, Urteilen und Entscheiden, Georg-Elias-Müller-Institut für Psychologie, Universität Göttingen

- Prof. Dr. Nivedita Mani, Free Floater Nachwuchsgruppe Spracherwerb, Universität Göttingen

- Prof. Dr. Michael Waldmann, Abteilung Kognitionswissenschaft und Entscheidungspsychologie, Georg-Elias-Müller-Institut für Psychologie, Universität Göttingen

- Prof. Dr. Uta Lass, Abteilung Experimentelle Psychologie, Georg-Elias-MüllerInstitut für Psychologie, Universität Göttingen

Tag der mündlichen Prüfung: 4. März, 2014 
My sister had a baby. We would have company over and she'd be there with her breast out, feeding him ... cereal or whatever.

The other day she took me aside and said 'Emo, can you baby-sit little Derek while I go to the carnival ... and look for the father?'

I said, OK. So I' $m$ pushing him through the park, and he's crying ... because I forgot the stroller.

I take him home and I'm trying to rinse out his diaper in the toilet [...] ... I accidentally let go of his foot. (Emo Philips, cited in Goatley, 2012) 


\section{Preliminary note}

The present dissertation is a publication-based (pseudo-cumulative) dissertation. It is based on one theoretical book chapter published in a collection of linguistic articles, on one original research article currently submitted and in revision for an international peer-reviewed journal, and on another original research article currently in preparation for submission to an international peer-reviewed journal.

Mayerhofer, B., \& Schacht, A. (2013). Salience, accessibility, and humorous potential in the comprehension of garden path jokes. In M. Dynel (Ed.), Developments in linguistic humour theory (pp. 341-366). Amsterdam: John Benjamins Publishing.

Mayerhofer, B. \& Schacht, A. (in revision). From incoherence to mirth: Neuro-cognitive processing of garden path jokes. Manuscript re-submitted for publication.

Mayerhofer, B., Maier. K., \& Schacht, A. (in revision). Investigating salience and accessibility: Priming effects on the interpretation of garden path jokes. Manuscript re-submitted for publication.

I extracted contents of all three articles and integrated them with each other. I served as first author in all articles. In particular, I was responsible for (i) developing the theory, (ii) designing and conducting the experiments, (iii) analyzing and interpreting the data, and (iv) writing up and publishing the manuscripts. Throughout this thesis I often use the pronoun "we", since my supervisor, Prof. Dr. Annekathrin Schacht, supported me with helpful advice and close collaboration throughout all of these stages. 


\section{Acknowledgments}

First of all, I would like to thank my supervisor, Annekathrin. Without her guidance, expertise, and support, this dissertation would not have been possible. She trusted me, had faith in this project, and was courageous enough to deal with my craziness and to engage in this interdisciplinary research project.

I also want to thank my family and my parents who have always supported my academical career. I am also grateful to my second adviser, Hannes Rakoczy, for his input and his encouraging interest in the project. I thank Marta Dynel for her editing effort and her helpful comments with two theoretical book chapters that were a result of my dissertation project. I want to thank Katja Maier for the reading time data collection and contributions to the stimulus material construction, Mareike Bayer for being very helpful with data analysis issues. I am grateful to Markus Steinbach who gave me helpful advice at the beginning of the project, and to Edgar Onea who was very helpful with his online rating tool OnExp. I want to thank all my colleagues in our research lab. It feels very good to work in a group with such a friendly atmosphere and quite regular mensa times. I want to thank all CRC members who gave me so much input over the last three years, and additionally Thomas Weskott for comments on the experimental design at the beginning of the project. Especially, I want to thank Katie von Holzen for being one of my biggest supports through the last three years. She helped me with proof reading, was a crying shoulder and a very suitable drinking companion at times. I also want to thank members all over the world from the humor research community who were inspiring and encouraging. I want to thank Daniele Panizza who was a big support in the final phase of the project, even though he introduced me to the "kill your 
p-values function" only two weeks before my submission.

I want to thank all my close friends, but especially Jonny, Ben, and Marcus. Even though we lived in different cities over the last years it was always very stabilizing to exchange outlooks on the current progress in life and career. I also want to thank all the participants who anonymously helped me a great deal by providing nice data. Last but not least, I am very grateful to Eric Moses who let me stay in his apartment in Vienna while he was away when I was writing the final paragraphs of this dissertation; I would also like to thank, anonymously, the owner of this apartment. You have a great record collection. 


\title{
Processing of Garden Path Jokes: Theoretical Concepts and Empirical Correlates
}

\author{
Bastian Mayerhofer \\ CRC Textstructures \\ Georg August Universität Göttingen \\ Göttingen, Germany \\ 2014
}

\begin{abstract}
In garden path jokes (GP jokes), an initially dominant semantic representation of an ambiguous text is violated. It needs to be revised in order to re-establish a coherent representation. The processing of GP jokes is modeled on a theoretical level as a dynamic interaction between a stimulus and a recipient. Both sides of the interaction are influenced by a wide range of factors that together determine the comprehension process. The focus is put on three aspects: (i) the salience of the first interpretation, (ii) the accessibility of the hidden interpretation, and (iii) the humorous potential of the whole joke. The comprehension process is assumed as probabilistic, non-monotonic, and incremental reasoning towards the most plausible interpretation of both linguistic and non-linguistic input. Basic assumptions about the sequential nature of the processing - detection of incoherence, revision of the semantic representation, and emotional reaction (mirth) - are empirically investigated in a first series of experiments. 48 GP jokes were manipulated and presented to participants in three conditions: (i) with coherent ending, (ii) with joke ending, or (iii) with discourse-incoherent ending. A rating study $(N=69)$, a reading times study $(N=24)$, and three studies with recordings of ERP and pupil changes $(N=21,24$, and 24) supported the hypothesized cognitive processes. Jokes were rated as more funny, moderately unpredictable, and comprehensible. They
\end{abstract}


showed increased reading times of the final word compared to coherent endings. ERP data mainly revealed semantic integration difficulties (N400). Some weak and mixed evidence is presented for an enhanced late frontal positivity (fP600), possibly related to the emotional outcome, and for a late left anterior negativity (LLAN), possibly related to increased processing effort. The emotional reaction to GP jokes is supported by larger pupil dilations in response to joke endings. Incoherent endings were rated as less funny, less predictable, and incomprehensible. They also showed increased reading times. The N400 was stronger and sustained. Incoherent endings also showed a context-sensitive P600 effect. An additional self-paced reading time experiment highlighted the influence of contextual constraint on the interpretation and re-interpretation processes by semantic priming prior to the GP joke. These findings support the theoretical conceptualization of salience and accessibility. Taken together, the processing of GP jokes is presented as playful communication that allows the strengthening of important cognitive skills related to the adaptation to changing environments. Mirth as the emotional reaction accompanying the cognitive processing is discussed as a reward for the engagement in this behavior. 


\section{Contents}

1 General Introduction . . . . . . . . . . . . . . . 1

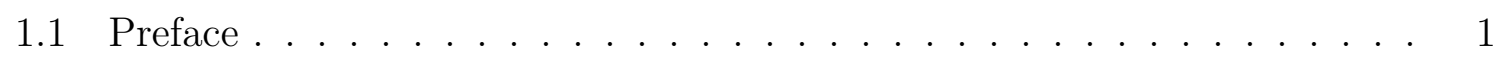

1.2 A Neuro-Cognitive Approach to Humor . . . . . . . . . . . . . . . . . . . 2

1.3 Discourse Comprehension . . . . . . . . . . . . . . . 8

1.4 Garden Path Jokes . . . . . . . . . . . . . . . . . . . . . . 10

1.5 Research Questions and Scope of the Dissertation . . . . . . . . . . 11

1.6 Outline and Methodological Approaches . . . . . . . . . . . . . . 12

2 Theoretical Concepts ........................ 13

2.1 Garden Path Joke . . . . . . . . . . . . . . . . . . . . 13

2.2 Probabilistic, Non-monotonic Models of Discourse Comprehension . . . . 18

2.3 Probabilistic Salience . . . . . . . . . . . . . . . . . . . . . . 21

2.4 Discourse Incoherence . . . . . . . . . . . . . . . . . . . . . . . . . . 27

2.5 Accessibility . . . . . . . . . . . . . . . . . . . . . 28

2.6 Humorous Potential . . . . . . . . . . . . . . . . . . . 32

2.7 Empirical Assumptions and Evidence . . . . . . . . . . . . . . . . . 37

2.8 Conclusion . . . . . . . . . . . . . . . . . . . . . . . . . 43

3 From Incoherence to Mirth . . . . . . . . . . . . . . 45

3.1 Introduction . . . . . . . . . . . . . . . . . 45

3.2 Experiment 1: Reading Times . . . . . . . . . . . . . 51

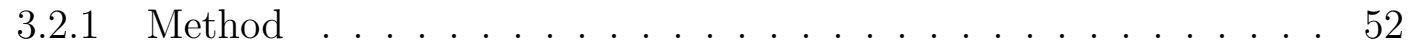

3.2.2 Discussion . . . . . . . . . . . . . . . . . . 57

3.3 Experiment 2: Evidence from ERPs and changes of pupil size . . . . . 57

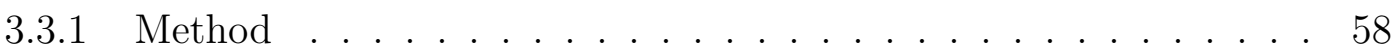


3.3 .2 Results . . . . . . . . . . . . . . . . . . . . 61

3.3.3 Discussion . . . . . . . . . . . . . . . . . . 65

3.4 Experiment 3: ERP Comparison between Joke and Coherent . . . . . . . 69

3.4.1 Method ...................... 69

3.4 Results . . . . . . . . . . . . . . . . . 70

3.4 .3 Discussion . . . . . . . . . . . . . . . . . . 72

3.5 Experiment 4: ERP Comparison between Incoherent and Coherent . . . 74

3.5.1 Method ........................ 75

3.5.2 Results . . . . . . . . . . . . . . . . 76

3.5.3 Discussion . . . . . . . . . . . . . . . . . 77

3.6 General Discussion . . . . . . . . . . . . . . . . . . 78

4 Investigating Salience and Accessibility . . . . . . . . . . 85

4.1 Introduction . . . . . . . . . . . . . . . . . . . . 85

4.2 Experiment 5: Contextual Constraint on Interpretation Processes . . . . 89



4.2 .2 Results . . . . . . . . . . . . . . . . . . 96

4.2 .3 Discussion . . . . . . . . . . . . . . . . 96

5 General Conclusion .................... 103

5.1 Empirical Evidence: Quasi-Replication and New Findings . . . . . . . . 103

5.2 Implications . . . . . . . . . . . . . . . . . . 106

5.3 Future Direction . . . . . . . . . . . . . . . . . . . . 112

5.4 Concluding Remarks . . . . . . . . . . . . . . . . . . . . . . 115



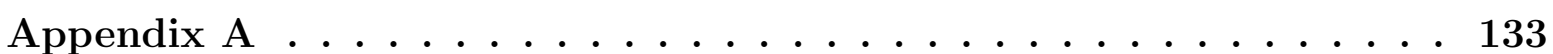

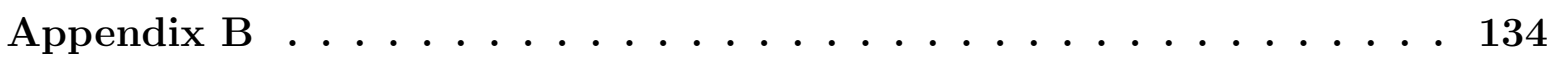

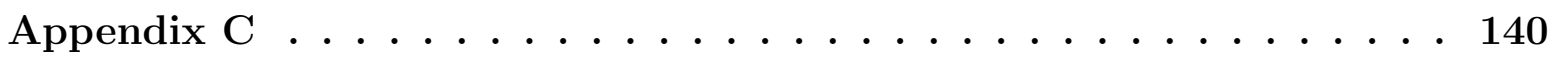




\section{List of Figures}

3.1 Box plot of the three scales of the ratings. Every data point is one observation of one participant and one stimulus. The thick line is the median, the box represents the $25 \%$ and $75 \%$ quantiles, and the whiskers are the minimum and maximum values, while points represent statistical outliers. . . . . . . . . . . . . . . . . 54

3.2 Difference maps of the conditions for the three time windows and grand average waves for selected electrodes as a function of Time relative to the stimulus onset and Condition in Experiment 2. . . . . . . . . . . . .

3.3 The grand average waves of the changes in pupil area (arbitrary unit) as a function of Time relative to the stimulus onset and Condition. The grey box indicates the time window with significant main effects of the running ANOVA for every $50 \mathrm{~ms}$. The arrows indicate the time windows with Bonferroni-corrected significant Post-hoc comparisons. . . . . . . . .

3.4 Scatterplot of the mean amplitude in the LLAN time window and its ROI and the total comprehension accuracy per participant split up for each condition in Experiment 3. Regression lines indicate the significantly negative linear relationship between these two variables. . . . . . . . . . . 72

3.5 Difference maps of the conditions for the three time windows and grand average waves for selected electrodes as a function of Time relative to the stimulus onset and Condition in Experiment 3. . . . . . . . . . . 73

3.6 Difference maps of the conditions for the three time windows and grand average waves for selected electrodes as a function of Time relative to the stimulus onset and Condition in Experiment 4. . . . . . . . . . . . . 78

4.1 (a) A schematic representation of the time course of the sequential presentation of a complete trial. (b) A screenshot of the self paced moving window presentation of the example stimulus in German at the reading position of PL. (c) A schematic representation of the design and the manipulations. Bold indicates a direct influence; non-bold indicates only an indirect influence. The arrows indicate an increase or a decrease on the salience/accessibility. 
4.2 Z-transformed mean reading times (trimmed and aggregated across participants and condition) of words as a function of Priming Condition and the three regions of interest: Set-up (reading time of the whole text fragment $=\mathrm{T} 1$ ), PL (final word $=\mathrm{T} 2$ ), and FS (average reading time of the words of the follow-up sentence $=$ T3) $\ldots \ldots . \ldots . \ldots 97$

5.1 Text and GP joke comprehension as interaction between a stimulus and a recipient. Theoretical concepts on both sides of this interaction and its empirical correlates investigated, supported, and suggested within the dissertation project. . . . . . . . . . . . . . . . 112 


\section{List of Tables}

3.1 Descriptive data of the matched stimulus features. . . . . . . . . . 53

3.2 Descriptive data of the behavioral variables in Experiment $2(N=21) \quad$. 62

3.3 Descriptive data of the behavioral variables in Experiment $3(N=24) \quad$. 71

3.4 Descriptive data of the behavioral variables in Experiment $4(N=24) \quad$. 76

4.1 Descriptive data of the reading times in Experiment $5(N=46) \ldots$. . 97

5.1 The most highly correlated variables of the data base. Correlations are based on the Spearman-method. . . . . . . . . . . . . . . . . . . 142 


\section{Chapter 1}

\section{General Introduction}

\section{$1.1 \quad$ Preface}

A comedian enters the stage and announces to the audience: "I want to die peacefully in my sleep like my grandfather. Not screaming in terror like his passengers." ${ }^{1}$ This grandfather joke exploits a mechanism that is a ubiquitous strategy of verbal humor. In this dissertation, this fairly homogenous subclass of verbal humor will be referred to as a garden path (GP) joke. One does not need to be a comedian to know this specific moment between the delivery of a joke and the mirthful reaction that hopefully follows it. There is a confused look in the faces that instantly changes to smile and laughter, once the joke is successfully comprehended. But what exactly is happening in the recipient's mind in this very moment between confusion and laughter? Theoretical considerations about the essential aspects of humor have a long tradition in the history of philosophy, aesthetics, literature, and even psychoanalytical psychology (see for a historical overview Martin, 2007, p.20ff.). The interest of empirical and cognitive psychology in the subject, however, still remains scarce. Investigating the underlying neuro-cognitive and emotional processes of this very moment can reveal important insights for at least two overlapping research fields: psychology of humor (Martin, 2007) and discourse comprehension (e.g., Kintsch, 1998).

\footnotetext{
${ }^{1}$ Attributed to Bob Monkhouse, retrieved from http://www.bitcomedy . co.uk/comedy-features/ 101-short-jokes/ on the 4th of June, 2013.
} 
The processing of verbal humor - in particular of GP jokes - can only be fully understood within the context of these two research fields. On the one hand, it is necessary to set out a framework of humor, specifically its comprehension by the human mind. On the other hand it deeply touches questions investigated in the field of text and discourse comprehension. Both research fields will be depicted in more detail in Chapter 2, but some important aspects will be described in advance here in the general introduction.

\subsection{A Neuro-Cognitive Approach to Humor}

The Oxford English Dictionary defines humor as:

that quality of action, speech, or writing which excites amusement; oddity, jocularity, facetiousness, comicality, fun. [...] the faculty of perceiving what is ludicrous or amusing, or of expressing it in speech, writing, or other composition; jocose imagination or treatment of a subject. (Simpson, Weiner, et al., 1989, p.468)

This definition (cited in Martin, 2007) presents three core aspects of humor: (i) a quality to be perceived as amusing, (ii) mental operations, and (iii) an emotional reaction (amusement, exhilaration, or mirth). Humor is associated with amusement. Amusement, on the contrary, is usually associated with humor. When trying to understand humor from a scientific point of view it is necessary to disentangle and clarify two important aspects related to humor. First, it is important to understand that humor, respectively the mental operations associated with humor, and laughter, respectively the emotional state of mirth, are overlapping and dynamically interacting, yet distinct phenomena. Humor can be present without the presence of laughter, just as laughter can be present without humor (see Gervais \& Wilson, 2005). 
Talking about the processing of verbal humor, one has to bear in mind that there are an emotional aspect and a cognitive aspect to be considered. These two aspects can be attributed to two distinguishable neural pathways. Processes related to the cognitive aspect mainly involve cortical and subcortical structures. Processes related to the emotional aspect mainly involve the mesocorticolimbic dopaminergic reward system and the amygdala (see for an overview Vrticka, Black, \& Reiss, 2013). As for the emotional aspect, it is further important to distinguish two kinds of laughter which are also based on dissociated neurological circuits, the so-called Duchenne-laughter vs. the non-Duchenne-laughter. Only the first one is laughter that is the expression of the emotional state called amusement, exhilaration or mirth. It is characterized by the crow-feet around the eyes, the muscular activation of the orbicularis oculi (see for a detailed description of the emotional state in Ruch, 1993). Gervais and Wilson (2005) have convincingly argued that the emotional state associated with Duchennelaughter evolutionarily originated in the context of play. Laughter communicates that an individual currently is placed in a safe environment. This emotional state is portrayed as highly contagious and associated with a wide range of evolutionary advantages on both the individual and group level.

In line with this idea, Fredrickson (1998) described positive emotions - as for example amusement, i.e. mirth - to be radically distinct from negative emotions. According to her "broaden-and-build" theory, positive emotions serve to broaden the scope of attention whereas negative emotions narrow down our perception in order to perform specific actions that guarantee survival; such as the fight or flight instinct in reaction to rage or fear triggering situations. This means that positive emotions are associated with a different cognitive style. This cognitive style is characterized by a stronger dissociation of goaloriented behavior (Apter, 1989). Adapting a playful state of mind enables to build up specific social, physical or cognitive skills that enrich the individual's resources. This hy- 
pothesis about the positive emotions was empirically quite well supported (Fredrickson \& Branigan, 2005). Participants whose affective state was changed by watching positive films (amusement or contentment) were more likely to apply a global span of attention in a visual processing task compared to participants who watched films with neutral or negative emotional content. The relationship between cognitive style and emotional state/mood can be found in a wide range of cognitive tasks (Clore \& Huntsinger, 2007), and is also very well corroborated by neuro-cognitive findings about the relationship between positive emotions, dopamine level, the activation of the mesolimbic reward system, and cognitive flexibility, such as creativity or problem solving (Ashby, Isen, et al., 1999; Ashby, Velentin, \& Turken, 2002). Positive emotions, therefore, seem to trigger a cognitive style that allows people to think outside the box, to recognize unexpected solutions or original relationships and associations. This in turn activates the mesolimbic reward center and increases the dopamine level in the brain, which additionally induces a positive mood. It makes sense to see this cyclic process as a specific mode of playful behavior to train and build personal resources which can only take place in safe (i.e. "non-threatening") environments (Gervais \& Wilson, 2005).

As for the cognitive aspect, it is important to understand the ontological status of humor (see for a detailed account on this question Hurley, Dennett, \& Adams, 2011, p.16). Is humor a real existing feature of the environment or is it a construction of the human mind? Unlike color, weight, or length, humor is not an inherent and intrinsic feature of certain stimuli in the environment. As a consequence, there is no objective measure of humor. Different individuals will end up with different judgements about the amusement of a specific situation, event, object, or joke. Nevertheless, there usually exists a huge overlap in the judgements about what people would consider as funny or amusing. This is especially true for people who share a common cultural or sociological background. But some stimuli also seem to be quite universally considered as humorous. Humor 
might best be understood as a latent potential that could express itself as humorous experience with the help of an individual's perceptual effort. Consequently, humor has to be considered as an interactional phenomenon between stimuli in the environment and an interpreting mind.

The question then arises as to which features a stimulus has to show in order to carry this latent humorous potential and to be (reliably) perceived as humorous by individuals. The most dominant answer within a framework of cognitive sciences can be traced back to ideas by Kant and Schopenhauer, and deals with different variations of the concept of "incongruity" (Forabosco, 1992; Giora, 1991; McGhee, 1979; Nerhardt, 1977; Suls, 1972). ${ }^{2}$ One basic assumption unifies the different theoretical proposals related to the concept of incongruity: Incongruity is proposed to arise due to a contradiction or violation between a mental prototypical representation of a stimulus or situation including its associated expectations - and its actual emerging appearance. The physically most vivid realization of this idea was carried out in an experiment by Nerhardt (1977). Participants were confronted with different weights that they had to lift. A series of weights served as the learning phase. By lifting one after the other participants started building up expectations about the range of weight these external stimuli maintain (mental prototypical representation). After this learning phase participants then lifted a weight that was much lighter or much heavier than the range of weight they had encoded as prototypical representations in the learning phase. The difference in weights accordingly could serve as a physical measure of incongruity. As hypothesized, participants who were confronted with a high incongruity, spontaneously reacted with laughter.

On a more abstract notion, Koestler (1964) defined incongruity as the "bisociation" of two incompatible ideas. As for the comprehension of metaphor or like any other cre-

\footnotetext{
${ }^{2}$ Other important families of theories of humor have been suggested in the literature but are not as relevant for this dissertation project. (see Martin, 2007)
} 
ative act of the human mind, humor is related to the mental ability of simultaneously maintaining and integrating two incompatible interpretations of a stimulus. Similar to the weight-experiment, this incompatibility is additionally accompanied by a strong contrast between these two ideas. These considerations have been continued and elaborated within the framework of "mental spaces" (Fauconnier, 1994), "conceptual blending" (Fauconnier \& Turner, 2008) and "semantic leaps" (Coulson, 2001). According to these approaches, humorous stimuli offer the possibility that semantic concepts that are originally unrelated to each other can be blended with each other. These new, unusual, and creative combinations of memorized chunks of knowledge from different domains are assumed to be at the core of a humorous experience.

Incongruity theories of humor have been criticized as being mere descriptive explanations of the features of a potentially humorous stimulus. They do not offer full theoretical explanations of why incongruity is perceived as funny (see Hurley et al., 2011, p.45ff.). A theory with higher explanational power that intends to connect the concepts of incongruity theories with its neuro-cognitive underpinnings and evolution-theoretically embedded conjectures was recently presented by Hurley et al. (2011). Their theory is based on the assumption that individuals on the one hand need to have stable and reliable beliefs and "theories" about their environment. These beliefs are usually unquestioned in order to allow individuals to function quickly and efficiently. On the other hand, individuals need to be able to adapt quickly and efficiently to new and unexpected changes in the environment. Accordingly, in a nutshell, these authors assume that humor has developed as an emotional reward for individuals in order to constantly question and overcome committed false beliefs before they enter the long-term memory as crystallized knowledge. Certain situations and stimuli lead individuals to an automatic and covertly entered false belief. Consequently, individuals then rely and act upon this false committed belief. Since such a behavior in the long-term means 
an evolutionary disadvantage, the detection and debugging ${ }^{3}$ of the cognitive representations of the environment is crucial. These debugging processes are highly costly and demanding for the cognitive system. The emotional benefits of a humorous reaction accordingly motivate an individual to carry out these adjustment processes to surprising new input. This "belief revision" concept is compatible with both outlined realizations of incongruity theories. On the one hand, it is able to explain on a more in-depth level why the detection of incongruity and its resolution by finding an alternative rule that accounts for the violation lead to the experience of humor. Incongruity-detection and -resolution are based on the debugging of a false committed belief regarding the current situation or environment. On the other hand, this approach is able to cover the concept of "bisociation" (Koestler, 1964), since the switching between two (or more) alternative interpretations of a specific stimulus taps into the same evolutionarily advantageous mechanism of quick adaptation and model-building of representations of an individual's environment. Last but not least, this theory is compatible with the outlined emotional aspect to humor. The advantage of a quickly adapting cognitive belief system can be considered as one - possibly the most important one - of the intellectual benefits that individuals gain from the playful behavior in a safe and non-threatening environment, as described above.

Summarizing the outlined concepts about the nature of humor, it is possible to portray at least one class of stimuli that carry certain features which are reliably perceived as humorous. They involve an incongruity-inducing mechanism, which as described is either related to the revision of a committed false belief during an interpretation process or to the simultaneous representation or alternating realization of at least two incompatible and contrasting conceptualizations of objects or situations (see Mayerhofer, 2013).

\footnotetext{
${ }^{3} \mathrm{~A}$ similar point had been put forward by Minsky (1984) who related humor to the censorship of erroneous reasoning.
} 


\subsection{Discourse Comprehension}

In popular culture, the following riddle (discussed in Carreiras, 1996; A. Sanford, 1986) became well known as the "surgeon riddle". It was subsequently taught to numerous students in the field of gender studies and related disciplines.

A young boy and his father are in a car accident. The father dies at the scene. The boy is transported to the hospital, taken immediately into surgery. But the surgeon steps out of the operating room and says, "I can't operate on this boy he is my son!" (unknown origin)

At least in earlier days, this text was accompanied by severe comprehension impedance. Many readers were incapable - or at least found it highly difficult - to retract the dominant interpretation that the surgeon was male and to substitute it with the alternative interpretation that the surgeon was female; i.e. the mother of the son. The example illustrates the importance of automatic imports of background knowledge during the comprehension of a discourse/text. Therefore, it served to deconstruct gender stereotypical knowledge in society. On a less sociological level, the surgeon riddle is an excellent example of the default interpretation processes necessary for a reader's comprehension of a chunk of words and sentences in order to build up a coherent mental representation of what the sender of the text planned to communicate.

Research on discourse comprehension focuses on the interplay between the linguistic input (letters, words, sentences, syntax, etc.), and how this input is incorporated by readers in order to build a cognitive representation of this input. It is generally agreed that the construction of this mental representation of a discourse is based on a complex integration of syntactical, lexical-semantic and referential properties of the linguistic input in combination with background knowledge and pragmatic aspects that take place 
in the social interaction between the sender and the recipient of a message (e.g, Graesser, Millis, \& Zwaan, 1997; Kintsch, 1988; A. J. Sanford \& Garrod, 1998; Zwaan \& Radvansky, 1998; Zwaan \& Rapp, 2006). However, language is only an approximative medium. Individual differences, vague denotations, ample room for fine-grained differences, diverse linguistic and non-linguistic backgrounds, and especially the impossibility of communicating every single detail of the message give space to much ambiguity as an inherent feature of language and communication.

Typically, the most basic goal of communication is the maximization of the overlap between the intended meaning of the message on the sender's part and the cognitive representation of the message on the recipient's part. Given that this maximization of the overlap between sender and recipient is the goal of a discourse, all participants of the discourse commonly put an effort into achieving this goal. This effort has been coined the "cooperative principle" of communication (Grice, 1975). According to this principle, both sender and recipient aim to reduce the ambiguity of the discourse. Communication, however, can additionally serve many other purposes. Most importantly in the current context, language is a suitable device for the purpose of a humorous experience. The sender of verbal humor often intentionally exploits pragmatic principles of communication. This type of communication has been referred to as "non-bona-fide" communication compared to "bona-fide" communication (Raskin \& Attardo, 1994). The humorous purpose of communication can be combined with any other functional aspect of communication. Humorous experience and social bonding are associated in certain types of teasing and playful-cooperative communication (e.g., Boxer \& Cortés-Conde, 1997; Kotthoff, 2006, 2009; Norrick, 2009). Humorous experience and critical deconstruction or the display of superiority and even hostility are usually combined in satirical or sarcastic usage of language (cf. the mechanism of irony as portrayed in Mayerhofer, 2013). 


\subsection{Garden Path Jokes}

A huge subclass of verbal humor, here referred to as garden path (GP) jokes (Dynel, 2009, 2012), directly illustrates the outlined theoretical considerations in both areas humor theories and discourse comprehension (see next chapter for a more detailed description). GP jokes are usually quite short texts that unify the outlined character of humorous stimuli with the exploitation of semantic-pragmatic discourse comprehension mechanisms. ${ }^{4}$ The "grandfather joke" of the general introduction can serve as an example of a GP joke. In this kind of joke, an initially dominant interpretation of an ambiguous textual input is automatically generated. The initially dominant interpretation turns out to be false at the ending of the text. New surprising linguistic input is presented. This input violates the semantic coherence of the discourse. The recipient has to find an alternative hidden joke interpretation that then allows to re-establish the semantic coherence of the text. In combination with some absurdity, or "inappropriateness" (Ritchie, 2004, p.64) of this hidden interpretation, this radical change of the mental representation triggers the emotional reaction amusement or mirth (Martin, 2007, p.8). The detection of the incoherence and the retrieval of the new semantic content that re-establishes the coherence of the text can be considered as processing equivalents of what is referred to as incongruity and incongruity-resolution stages in humor theories (e.g., Suls, 1972). In agreement with the outlined theories on verbal humor, the neuro-cognitive processing of GP jokes is hypothesized to follow a sequential process: (i) automatic interpretation that relies on a committed false belief, (ii) the detection of the violation of the coherence, (iii) re-interpretation and re-establishment of the coherence, and (iv) emotional reaction. This sequential process will be at the core of this dissertation project. It will be investigated theoretically and empirically.

\footnotetext{
${ }^{4}$ The basic GP mechanism, however, can be implemented also by longer texts, discourses, or even novels and films, not necessarily restricted to short jokes.
} 


\subsection{Research Questions and Scope of the Disserta- tion}

Both fields of research humor comprehension and discourse comprehension deal with a complex interaction between a stimulus and a recipient who perceives and elaborates this stimulus. Accordingly, an investigation of the processing of GP jokes needs to account for factors on both sides. On the stimulus side there are certain inherent linguistic and humor-specific features. These factors manifest themselves in a recipient's mind. The recipient draws automatic default inferences, aims to maintain a coherent representation, detects the incongruity, resolves the incongruity, and elaborates (Wyer \& Collins, 1992) the humorous potential. On the recipient side, certain inter-individual and intra-individual characteristics presumably determine how the stimulus features are perceived and processed.

This dissertation is an interdisciplinary project at the interface of cognitive psychology and cognitive linguistics. The processing of GP jokes will be approached by theoretical elaborations of the underlying concepts and by experimental-empirical investigations of possible correlates and measure variables of these underlying theoretical concepts. For both sides empirical correlates of the theoretical concepts are investigated. The investigation embodies an explicit focus on a subclass of verbal humor. This focus allows more specific conclusions. The aims of the project accordingly are: (i) theoretical elaboration of the hypothesized processing of verbal GP humor. This theoretical approach includes the adaptation of existing theories about verbal humor and the development of a theoretical and methodological tool box for the investigation of verbal GP humor; (ii) finding empirical evidence for the hypothesized processing of verbal GP humor. This empirical evidence consists of quasi-replication and refinement of previous findings for highly comparable stimulus material, and of new evidence related to new research designs and 
new methods.

\subsection{Outline and Methodological Approaches}

This thesis will be structured based on three independent manuscripts. One has been published, one is currently under revision in a peer-reviewed journal, and one is in the final preparation for submission to a peer-reviewed journal. The second chapter is an extraction of a theoretical contribution in a collection of linguistic articles called "Developments in linguistic humor theories" edited by Marta Dynel. Three important concepts related to the processing of GP jokes are depicted and developed within a neuro-cognitive and probabilistic frame work of discourse and humor comprehension: salience, accessibility, and humorous potential (Mayerhofer \& Schacht, 2013). The third chapter is a report of a series of empirical experiments that investigated the sequential nature of the neuro-cognitive processing of GP jokes. The joke processing is compared to two manipulations of these jokes. These manipulations resulted in coherent texts on the one side and totally incoherent texts on the other side. The report describes the stimulus material, a self-paced reading time study, and three ERP studies. The first ERP study also included the investigation of the pupillary response. The report embodies partly a quasi-replication of previous findings and partly presents new experimental data that can shed light on the general nature of the comprehension process of GP jokes. The fourth chapter is a report of a self-paced reading time study in which contextual constraint on the processing of GP jokes was manipulated by a priming paradigm. This design allowed the investigation of theoretical concepts which are depicted as determining factors on the processing of GP jokes in the second chapter. In the final chapter, the implications of the theoretical elaborations and empirical findings will be discussed. 


\section{CHAPTER 2}

\section{Theoretical Concepts}

\subsection{Garden Path Joke}

Text comprehension is a peculiar phenomenon. Given the complexity of the dynamic interplay between linguistic input, world knowledge, contextual knowledge, and inferential and internal monitoring processes, it is impressive that different individuals end up with mostly similar mental representations out of an accumulation of letters, words, and their grammatical realizations. Verbal humor in general, but in particular, a phenomenon, which can be referred to as garden path (GP) joke, exploits this relationship in such a way that it offers a very suitable field for the study of discourse comprehension processes.

Suls's (1972) two stage model of humor depicts a general mechanism. According to this model, expectations built up by a recipient of a humorous discourse are violated. If the recipient finds a rule that accounts for the violation of the expectation, the incongruity can be resolved. This sequential process of incongruity and resolution is supposed to trigger the humorous reaction. The two-stage model is very general and is claimed to underlie all jokes. However, humorous texts are heterogeneous and show various mechanisms, one of which is the GP mechanism. The GP mechanism, despite varying terminology, has been implicitly mentioned by several authors in the field of humor research (e.g., Dascal, 1985; Raskin, 1985; Attardo \& Raskin, 1991; Norrick, 2001). Other authors have explicitly focused on the GP mechanism and suggested a specific 
classification of this type of joke (e.g., Dynel, 2009; Hockett, 1973; Yamaguchi, 1988). In its basic form, the GP mechanism could be described as follows: an ambiguous setup of a text, usually the beginning of the text, lures a reader into building up one predominant interpretation. The expectations and mental representations according to this interpretation are subsequently violated at the punch-line, which is usually at the end of the text. The punch-line refers to the cue in the text that signals the reader that the current interpretation is not correct. Here, the reader has to overcome the incoherence of the text. The initially predominant interpretation of the set-up gets replaced by an alternative, hidden interpretation of it. Ritchie (2004, p.61) introduced a very helpful formalization and terminology in his so-called "forced re-interpretation model". According to his suggestions, important elements in this type of jokes are: (i) an initially dominant interpretation of the set-up (SU1), (ii) an alternative interpretation of the set-up (SU2), (iii) the meaning of the punch-line (PL), (iv) an interpretation (I) attained by integrating PL and SU2. The relationships between these elements and some further notions will be adapted probabilistically in the present account. In terms of their structure, GP jokes are similar to the well-studied GP sentences. In the latter, the initial interpretation turns out to lead to a syntactical ungrammaticality. In GP jokes, the whole discourse is incoherent on a semantic or pragmatic level as long as the initial interpretation is not substituted by the hidden joke interpretation. GP jokes are semantically incongruent according to the recipient's mental representation, which is constructed during the comprehension of the text. This difference between GP sentences and GP jokes will be demonstrated in the following examples.

(1) The boat floated down the river sank. (Bever, 1970) (GP sentence)

(2) The boy hit the girl with the glasses. (Syntactically ambiguous sentence, sometimes also referred to as a GP sentence)

(3) The boy hit the girl with the glasses. He wished he had had a base-ball 
bat instead of the glasses as a weapon. (GP joke based on parsing 1)

(4) The boy hit the girl with the base-ball bat. Then, he ran away because he was afraid of her base-ball bat. (GP joke based on parsing 2)

(5) A lady went into a clothing store and asked: "May I try on that dress in the window?" "Well," replied the sales clerk doubtfully: "don't you think it would be better to use the dressing room." (GP joke taken from Ritchie, 2004)

In (1), the initially dominant syntactic parsing is an active construction. The occurrence of the final verb forces a reader to re-organize the syntactical parsing by understanding that "floated" appears in the function of a participle. It introduces a reduced passive relative construction rather than serving as the verb of the main clause. This is discovered only at the end when one reads the final verb "sank". In order to understand the sentence as a grammatically correct one, the recipient is forced to reprocess the initial parsing. Here, the changes are quite restricted to the syntactical level. The syntactical changes might also alter the semantic representation. In this example, one needs to enrich the representation by an unknown subject that is needed to "float down the boat". However, the reanalysis only leads to a minor change of the recipient's mental representation.

Other types of syntactically ambiguous sentences, as Example (2) or the customer's question in Example (5), are sometimes also called GP sentences even though there is no ungrammaticality involved. A reader is not forced to re-process the syntactical parsing. These phrases are only syntactically ambiguous. In (2), the context should bias the recipient towards a parsing which attaches the noun phrase ("the glasses") to the girl because of background knowledge. It seems more plausible that glasses are used as a seeing aid than as a weapon. Dynel (2009, p.129) argues that syntactical 
ambiguity is not well suited for the creation of GP joke-induced humor, probably due to the lack of semantic contrast between two syntactical interpretations. This contrast is supposed to be necessary for the incongruity; hence for the humorous potential. She claims that syntactical ambiguity is not conducive to a GP joke unless it is "intertwined with lexical ambiguity" (Dynel, 2009, p.130). More generally, however, syntactically ambiguous sentences are suitable for GP jokes, if the initially predominant representation - based on the initially dominant parsing - becomes incoherent on a semantic level of the discourse, as in the reply of the sales clerk in (5). Also (2) becomes a GP joke, a mildly amusing one admittedly, if one adds a sentence as in Example (3). This sentence makes the discourse incoherent according to the initially dominant interpretation. The comprehension involves a change in the mental semantic representation of the discourse.

Comparing the GP sentence (1) with the GP jokes (3), (4), and (5) reveals that in the latter examples the violation occurs on a semantic level. Example (1) is a syntactically incorrect sentence until the syntactical parsing is updated. In the GP jokes $(3,4$, and 5), all sentences remain syntactically correct even if one does not re-organize the initial parsing of the first sentence. However, the semantic violation triggers discourse incoherence, and this incoherence forces the reader to resolve it. The ambiguity of a GP joke can occur on various linguistic levels. Some kinds, e.g. phonological, referential, pragmatic (based on presupposition or implicatures) ambiguities, appear more often than others (see Dynel, 2009, p.117ff., for a detailed classification of different types of ambiguity in GP humor).

One important observation, described in more detail by Ritchie (2006), is that sometimes the reader is directly led up the garden path as in (3), but in some cases, the reader is only indirectly led up the garden path. In the latter instances, the reader follows a character in the story with the wrong interpretation, as in (5). In other jokes, only a character is led up the garden path but the reader is not. Here, the humor arises due to the discrepancy 
between the knowledge of the reader and the character's false belief in a story. The term GP joke should not cover these latter instances. The distinction can be very difficult, as one might realize if one analyses (5) very carefully. In this example, the ambiguity arises on a more complex level, namely on the assumption that the sales clerk would parse the customer's syntactically ambiguous question according to the most dominant parsing, as probably does the reader of the story. The story, however, carries the possibility that the sales clerk intentionally or unintentionally misunderstands the customer and responds, following the less salient syntactical parsing. Therefore, the discourse incoherence in the joke is not primarily due to the ungrammaticality of the salient parsing structure of the question. The sales clerk's answer rather violates the assumption that the sales clerk understands the customer correctly and behaves ordinarily. The resolution of this incoherence is based on the interpretation of the whole discourse. One has to realize that the sales clerk misunderstood the customer's syntactically ambiguous question.

Dynel (2009), building up on the graded salience hypothesis by Giora (2003) and on Levinson's work of presumptive meanings (2000), points out two important features of GP jokes: (i) the salience of the initially dominant interpretation and (ii) the "cancellability" of this initial interpretation. Salience refers to the fact that one interpretation of an ambiguous (linguistic) input is preferred automatically, that is without effort and is made by default. Cancellability refers to the possibility of retracting the initial interpretation in the light of contrary textual input, i.e. the violation of the coherence of the discourse as in GP jokes. Both factors are related to the initially dominant interpretation. For the understanding of GP jokes, however, it is essential to focus also on the accessibility of the non-salient, hidden meaning. The hidden interpretation has to be activated in order to resolve the discourse incoherence. Accessibility, as a connection between human memory structures and language comprehension, has been applied to the comprehension of humorous riddles (De Palma \& Weiner, 1992). To our knowledge, 
so far, it has played no explicit role in the research on GP jokes. Accessibility, in the current context, refers to the difficulty of finding the alternative interpretation. Referring to the examples again: on the one hand, the hidden interpretation of (3) and (4) seems to involve less inferential processes than the one in (5). Examples (3) and (4) only

demand new syntactical parsing, whereas (5) requires the higher-order representation of the false-belief of the sales clerk's wrong syntactical parsing. On the other hand, it might be less straight-forward to think of the use of glasses as a weapon than to think of a naked woman in a shop window. The latter could make the alternative syntactical parsing in (5) more easy than in (4). As a result the overall-accessibility for (5) could nevertheless be higher than for (4), despite the higher number of necessary inferential steps.

\subsection{Probabilistic, Non-monotonic Models of Discourse Comprehension}

As any other phenomenon of perception and interpretation, language processing can be understood as a stochastic process; i.e. it involves an intuitive engagement of knowledge about probabilities and statistical properties of linguistic elements. One constantly needs to solve a problem to choose from a (theoretically) infinite number of possible interpretations for a given textual input only one interpretation which seems the relevant one given the input, the context, and the recipient's and the speaker's background knowledge. Considering all possibilities carries the risk of combinatorial explosion. Thus, a recipient usually settles on one interpretation unless there is no good interpretation to be found or unless there are two or more competing interpretations with equally balanced plausibility (see Hurley et al., 2011). A text is never ready-made but only delivers material for the construction of a supposedly intended meaning. For example, beginning 
a story with "There were a dog and a cat in the living room ..." could activate a wide range of possible semantic representations. On a spatial level, they could sit peacefully next to each other. The cat may be sitting on the dog, the dog may be sitting on the cat, the dog may be chasing the cat, or the cat may be chasing the dog. The list can be quite arbitrarily continued with compatible interpretations. However, there seems to be a decreasing order of plausibility, which can be formulated in terms of probabilities. The default interpretation seems to be a combination of the most prototypical situation including a cat and a dog. It is retrieved from knowledge and might involve pragmatic inferences (e.g., that the very small dog is sitting on the huge cat would be such a remarkable situation that one might assume that it should have been made explicit by a cooperative speaker). This knowledge is combined with the context of the utterance (if I just had watched a documentary about the cruelty of animals, the chasing interpretation might have received a stronger plausibility due to some priming effect of availability).

Ambiguity is a ubiquitous feature of language and texts. Yet, communication usually succeeds surprisingly well, and ambiguities are resolved very fast and correctly, i.e. in the way intended by a speaker. The probabilistic turn in competence models of the psychology of human reasoning (Oaksford \& Chater, 2007) has also affected cognitive linguistics. Probabilistic models of language processing are rising in popularity (Jurafsky, 2003; Chater \& Manning, 2006). In particular, Bayesian belief nets or networks appear to be a fruitful field for the future of probabilistic language processing.

Recall that the advantage of a Bayesian approach to language processing is that it gives a model of what probability to assign to a particular belief, and how these beliefs should be updated in the light of new evidence. (Jurafsky, 2003, p.30)

Bayesian networks are a model for knowledge representation with directed a-cyclic 
graphs. Nodes, representing specific events, are connected by edges. The edges between these nodes represent a conditional dependency between the nodes. The strength of the dependency is measured as a subjective probability assigned to it. Consider the classic "Tweety example" as in (6).

(6) Tweety is a bird. Therefore, Tweety can fly.

The underlying knowledge which allows the hearer to conclude that Tweety is able to fly can be formulated as a conditional event with a subjectively assigned probability. If $x$ is a bird $(A)$, it (normally) can fly $(B), P(B \mid A)=.95$ for example, read as the probability of $B$ (ability to fly) given $A$ (being a bird) as predicates of the entity $x$. Tweety, however, might be a bird which was born without wings. Tweety, for any reason, may have never learned how to fly. One can never be absolutely sure. But one can build up coherent beliefs of the events unfolding in the environment and assign subjective probabilities to them. The epistemic caution about Tweety's ability to fly is reflected by a probability that is slightly lower than 1 . The probability of .95 indicates a very high degree of belief (cf. De Finetti, 1970). But it is not a certain fact. If I find out that Tweety is a penguin, however, the new evidence changes the picture. This information might have been intentionally withheld by the communication partner. The new premise has to be integrated within the discourse representation. Belief revision occurs and the result of it is a much lower probability for the conclusion about Tweety's ability to fly (see Pfeifer \& Kleiter, 2010, for a detailed account of the Tweety example), $P$ ( $x$ is able to fly $\mid x$ is a bird $\wedge x$ is a penguin $)=.01$, for example; read as the probability of $x$ 's ability to fly given that $x$ is a bird and that $x$ is a penguin. The probability is not 0 because one might think of a super-penguin with the superpower of flying.

A probabilistic model for discourse comprehension by applying the idea of Bayesian networks offers several suitable characteristics: (i) it is incremental; i.e. new input can 
be integrated as soon as it emerges (on-line); (ii) it is non-monotonic; i.e. previously drawn conclusions and interpretations can be retracted in the light of contradicting input; and (iii) it is independent from the type of knowledge which has to be engaged. Linguistic knowledge like the frequency of specific parsing structures or concepts and categorization in the mental lexicon can be formulated in the same way as contextual background knowledge, social knowledge, or pragmatic knowledge. They can all be formulated as probabilistic conditional dependencies. It does not mean that a recipient of the discourse constantly and explicitly calculates probabilities with precise numbers. The claim is, however, that a human mind carries out these stochastic processes in an intuitive and unconscious way. Our Tweety example does seem structurally similar to a GP joke. A default interpretation of the premise is exploited in order to trick the recipient into inferring an incorrect conclusion, if Tweety turns out to be a penguin. One will certainly not burst out into laughter but some mild forms of amusement and the acknowledgement of a witty cognitive experience might occur, and it seems plausible to create a GP joke based on the "Tweety components".

\subsection{Probabilistic Salience}

As described in the previous section, language processing, to a great extent, has to deal with uncertainty, vagueness, and ambiguity. The resolution of ambiguity is still a highly debated issue. The main questions are: firstly, the time course of alternative activations (sequential or parallel) and, secondly, the different weights of the influences biasing a recipient towards one of the alternatives. Are these impacts stronger by linguistic features than by contextual factors, or vice versa, or are they equally important? The Graded Salience Hypothesis (Giora, 1997, 2003) assumes that language expressions carry "degrees of salience". This means that in the case of meaning construction based 
on an ambiguous utterance, the preference of one meaning over the other is graded, according to different linguistic factors. Context factors play a minor role in this account. Four main factors have an impact on the degree of salience of one interpretation: conventionality, frequency, familiarity, and prototypicality (stereotypicality) of the linguistic expression. In the case of GP jokes, this seems very plausible if they rely on ambiguities related to the mental lexicon. In many cases, the salience of one meaning is not as clear because it is a more complex chunk of words. Dynel (2009) additionally subscribes to the possibility of priming effects by the context or co-text. According to Dynel's account, the salience in GP jokes, which do not rely on a lexical ambiguity, can be described within models of "default reasoning" (Bach, 1984) and "presumptive meanings" (Levinson, 2000). These models assume, just as in the present account, that in the absence of clear evidence for one specific interpretation of a communicative act, recipients activate default interpretations. Default interpretations are the interpretations which involve the lowest processing effort. They do not need conscious inferential effort. Nevertheless, pragmatic inferences have to take place. This concept of default reasoning is crucial. The question, however, remains by which precise cognitive mechanisms one interpretation suddenly pops up as the effortless first conclusion. Probabilistic competence models of human reasoning may offer an answer to this question. In the case of GP jokes, it would be helpful to adapt a model that can account for a wide range of ambiguity types in a straightforward manner. In addition, the model has to be incremental and non-monotonic, in order to account for the flexible and quick on-line interpretation and re-interpretation processes during discourse comprehension.

Among many other linguistic phenomena, syntactical ambiguity, such as in GP sentences, has successfully been studied within the framework of probabilistic constraintsatisfaction models (MacDonald \& Seidenberg, 2006; Seidenberg \& MacDonald, 1999). Traditionally, the preference for one interpretation of the syntactical ambiguity has 
been described by the structurally based heuristics of the garden path model (Frazier \& Clifton Jr, 1997; Frazier \& Fodor, 1978). The heuristics are "minimal attachment", i.e. the reader prefers the simplest, the least demanding parsing structure, and the "late closure" approach, i.e. new words or phrases are attached to the actual clause. As a consequence of these heuristics, only one interpretation is maintained at a time. This leads to a sequential model of ambiguity resolution. Much empirical research on GP sentences, however, supports a multi-faceted account, in which several linguistic and non-linguistic cues are integrated in a very fast and dynamic way. These cues lead to constraints on the syntactical parsing, based on probabilistic knowledge (for a review see MacDonald \& Seidenberg, 2006).

Probabilistic models allow quite precise empirical predictions and can also be implemented on a computational level. For example, Jurafsky (2003) successfully applied a Bayesian model to sentence processing of syntactical ambiguity. The model took into account several different factors known to have an impact on the dominant main clause parsing of a GP sentence. The model succeeded on a computation level, as well as on an empirical level by predicting human processing of these sentences. A further advantage of probabilistic models of ambiguity resolution is that they can be applied to various kinds of ambiguities. Therefore, they can also be applied to GP jokes in which no structural heuristics can be used, since the ambiguity is not based on the syntactical parsing.

How can the mind be so easily tricked to stick and commit to one interpretation? One can hardly succeed in resisting the default reasoning. Hurley et al. (2011) offer a neurocognitive theory on humor which can shed light on this question. In order to understand the necessity of default reasoning and salient interpretations in the form of committed beliefs, they claim, one needs to dive into the mechanisms of the human cognitive system and the way knowledge might be stored and used for the purpose of selecting beliefs 
about the individual's environment. Humans constantly face a difficult problem, namely to find the optimal balance between two essential skills in evolution. On the one hand, individuals have to be able to carry out actions, decisions, and judgments quickly and correctly by relying on their interpretations of the reality and the conclusions drawn, based on these interpretations. On the other hand, humans need to be able to adapt very flexibly, fast, and correctly to new unexpected evidence; especially if this new evidence is contrary to the committed beliefs and interpretations they have based their actions and decisions on. Failing to do so would impede learning; and in the long term, the ability to survive. Hurley et al. (2011) argue convincingly that humor has developed as an emotional reward for human beings to overcome covertly entered false beliefs. These erroneous, but committed beliefs should be recognized before they become longterm memory structures. If they were not abandoned, this would make it even more difficult for the next time to build up the correct interpretation. The emotional benefits of a humorous experience motivate individuals to engage in the demanding activity of debugging the interpretational system. This effort is not pleasant, but the emotional reaction to humor makes it a pleasant experience.

This principle of committing to one interpretation but being flexible enough to change the interpretation in the light of new evidence also applies to communication, and GP jokes are a good illustration. Facing an ambiguous linguistic structure, the hearer of the utterance has to decide on one interpretation and commits to it. Otherwise, due to the infinite interpretative alternatives, communication would not be possible. One could not take for granted anything. If the recipient has to commit to one interpretation for the sake of mutual understanding, the best guess is the most probable/most plausible interpretation given the factual linguistic input, the co-text, the back-ground knowledge (including familiarity, conventionality, prototypicality, frequency, genre-specificity, etc. of a given utterance), empathic representation of the speaker's mind, pragmatic infer- 
ences, and the context of the utterance. Therefore, the salience of an interpretation might best be modeled as a probabilistic function of these factors. This can also explain why the salience of an interpretation is not only dependent on specific individuals, but also on context, linguistic and non-linguistic knowledge, and might even vary in one individual from one case to another. A set of alternative interpretations can be ranked by their assigned probabilities. Only the one with the highest ranking is entertained. It is the only interpretation the recipient is consciously aware of, whilst there might be other interpretations that are activated (at least to a small degree) but a recipient is not consciously aware of them. In the case of equally probable interpretations, one might not be committed to one interpretation but might oscillate between one and the other, or possibly remain undecided. However, one interpretation has to come first. By analyzing Example (7), I will try to exemplify the probabilistic salience of the first interpretation. In the same way as in the "Tweety example", it is possible to capture the knowledge engaged for the interpretation of the semantic features of the non-explicit character of the utterance (7).

(7) "Mummy, I just turned 14 years. May I please, finally, be allowed to wear a bra and make up?"

This utterance is very unspecific about the location, the time, and the characters involved in the story. Most of all, however, the ambiguity is the underspecification of the character's identity. The character's identity has to be enriched by the reader of this story. Background knowledge can be formulated again as probabilistic knowledge in the form of a conditional probability. A 14 year-old character $x$ is expecting to wear a bra and make-up. The most likely interpretation based on a typical recipient's probabilistic knowledge is that $x$, the unknown speaker of the utterance is a girl by the following (automatic) inference: If $x$ expects to wear a bra and make-up $(A), x$ usually is a girl $(B)$, 
$P(B \mid A)=.8-.97 .^{1}$ The strength of the degree of belief might depend on the recipient's stereotypical gender knowledge. There is an infinite number of other possibilities. $x$ could be a boy, it could be a speaking dog, it could even be an alien. In order to avoid a combinatorial explosion of infinite possibilities, the cognitive system has to take it for granted that the most probable interpretation is the one intended by the speaker of the discourse. This interpretation leads to a semantic representation of a girl talking to her mother, the salient interpretation.

However, the main contextual information here is that this example is printed in a scientific article about GP jokes. This context could influence the reading. The reader suspects that the utterance aims to lead him/her up the garden path. This skepticism could also be accounted for in the model. Given the expectation $(A)$ of $x$ and the contextual information $(C)$, namely a scientific analysis of GP jokes, the probability of $B$, i.e. $x$ being a girl, might be assigned a lower probability. Let us say: $P(B \mid A \wedge C)=.7$. Someone may even imagine having read a newspaper story about transvestites just before reading this chapter. This might have affected his/her knowledge, permanently or temporarily in such a strong way that he/she does not believe gender stereotypes. If the recipient also assumes that the person telling him/her this story shares the same knowledge, then the expectation of wearing a bra and make-up is not a strong cue for $x$ being a girl at all. Therefore, the probability of the interpretation of $x$ being a girl would be very weak, and the recipient would not commit strongly to this one interpretation.

\footnotetext{
${ }^{1}$ All probabilities are subjectively assigned, and therefore quite arbitrary numbers that could be more or less similar to the ones of possible readers. However, note that a basic assumption of Bayesian networks is that different individuals have different assignments of probabilities.
} 


\subsection{Discourse Incoherence}

If a GP joke works, the recipient has decided on one salient interpretation and has accordingly built up an internal representation of the scenario in the text. If nothing in the discourse forces the reader to re-think the initially salient interpretation, there is no need to doubt the correctness of this most probable interpretation; just as someone commits to the belief that the sun will rise every day in the morning given that the sun so far has risen every morning. That the sun would stop rising would qualify as unexpected new evidence, which is not coherent with the person's beliefs. It seems to be a core feature of the human mind that a violation of an established internal representation of the events in the environment, based on the theories and knowledge one has gathered, forces one to re-arrange the cognitive representation of the events. This innate need for coherence also applies to communication. Faced with the answer to the question of the (presumably) 14-year old girl, as in (8) the reader might have a similar experience.

(8) "Mummy, I just turned 14 years. May I please, finally, be allowed to wear a bra and make up?" -"No, and eat up your soup, my son."

The punch-line $(\mathrm{PL})$ is not coherent according to the default interpretation of the set-up, namely that the character in question is a girl. The discourse becomes incoherent for a moment, formulated as a conditional probability: $P(S U 1 \mid A \wedge P L)<.1$. Again, the probability of $S U 1$, namely that $x$ is a girl, becomes very low, given $A$ that $x$ expects to wear a bra and make-up, and given the $P L$ that the mother calls $x$ "son". The low probability of the interpretation forces one to abandon some of the underlying committed beliefs which render the discourse incoherent. Two important factors might influence the degree of incoherence. One is the resulting probability or plausibility according to 
the knowledge when trying to integrate the new evidence. The other is the possibility of "explaining it away" by adapting some auxiliary assumptions. For example, one way to keep the interpretation of $x$ being a girl could be to assume that for some reason the mother just likes to call her daughter "son", which is not usual but still possible. In such a case, the committed belief would not be altered. The core of the initial interpretation remains untouched. The recipient integrates the new evidence without retracting the predominant interpretation, but the resulting discourse remains incoherent to a certain degree. More likely, however, is another option: the incoherence of this discourse would trigger the search for alternative interpretations if the degree of incoherence would reach a certain threshold.

\subsection{Accessibility}

The third important feature in the comprehension of a GP joke is the accessibility of knowledge which enables the retrieval of at least one alternative interpretation in order to re-establish a coherent discourse. The concept of accessibility was mentioned by De Palma and Weiner (1992) for the comprehension of humorous riddles which were based on lexical ambiguity. The authors explicitly argued in favor of a relationship between accessibility and knowledge representation, even though their account was limited to lexical knowledge. Analogously to the degree of salience, accessibility can be thought of as a graded phenomenon. This should become intuitively clear by comparing the "mummy" joke with Example (9):

(9) "Susie, why do you feed your cat with seeds?" -"Because seeds are the favorite food of my bird." 
Example (9) is probably best classified as a joke which exploits the crossroad mechanism (Dynel, 2009, p.29ff.). In this kind of joke, no rational interpretation of the set-up is likely to be found by a recipient. Therefore, no default reasoning takes place, and the recipient has to await the information at the punch-line for the establishment of a coherent discourse. There are cases in which the mechanisms may overlap (Dynel, 2012). In this joke, the set-up contains incomprehensible elements. Susie's absurd behavior creates the incoherence already during the set-up of the text. This incoherence cannot be resolved without the help of the punchline. Therefore, no clear initially dominant interpretation can exist. However, at least some inferences are made by default even though they do not lead to a totally coherent interpretation of the discourse. The speaker of the question is assumed to be an adult, and Susie is accordingly assumed to be a child, for example. One basic assumption is that Susie feeds the cat with the intention of feeding the cat (SU1). This assumption is default and automatic. It is the most plausible interpretation given the textual input of the first speaker's question $(A)$. The final word, bird, which serves as the punch-line ${ }^{2}(\mathrm{PL})$ semantically violates SU1; formulated as a conditional probability, $P(S U 1 \mid A \wedge P L)<.05$, the probability of the first interpretation given the character's question and the PL becomes very low, leading to additional incoherence. Example (9) is not a textbook GP example. The GP mechanism, however, is partly involved. One of the default assumptions, namely the

\footnotetext{
${ }^{2}$ There seems to be confusion about the precise span of linguistic elements which form the PL. This question could possibly be answered by substitution tests. Instead of "bird", there could be "cat", and that would change the whole text into a non-joke. Changing "bird" to "my tweety", or "my canary", or maybe even to "my mouse" etc. would not alter the joke. The substituting concept has to be compatible with the representation of Susie's belief about a small pet being alive in the belly of the cat. "My monkey" would not work. If the utterance were changed into "My bird is hungry", the text would still be pretty much the same joke. These arguments speak for a locally restricted conceptualization of the PL. On the other hand, the full utterance must be processed for the message to arise completely. Accordingly, the whole final utterance should be considered the PL. Nevertheless, the recipient might already be able to understand the joke as soon as the word "bird" emerges even if it were at the beginning of the utterance. Such a position of the PL would probably just destroy the tension and the timing of the joke. Psychologically, "bird" is the precise element which adds the incoherence and which triggers the retrieval of the alternative interpretation. So, even though the whole utterance can be considered the PL, "bird" (or one of its compatible substitutes) is the crucial disambiguating element.
} 
one about Susie's intention turns out to be false. Independently from the classification of the joke, the re-establishment of a coherent semantic representation in this example involves a more complex, effortful, and inferential retrieval of knowledge than in other examples: Susie is a child, children have limited knowledge about biology, cats eat birds, the cat ate the bird, the bird is in the belly of the cat, Susie has a false belief that the bird is still alive in the belly of the cat. SU2, "Susie wants to feed her bird but it is dead because it was eaten by the cat", is the most probable interpretation given A and PL. The retrieval of the knowledge requires more inferential steps and higher-order belief representations. Therefore, the hidden interpretation might be much harder to find than that in Example (8).

The degree of accessibility refers to the ease of retrieving the hidden interpretation in order to overcome the incoherent discourse. It can be described as a function of several multi-dimensional factors. On the side of textual features, probable influences on the accessibility are: (i) the number of necessary inferential steps, (ii) the involvement of mental representations of false beliefs or different perspectives, (iii) the semantic distance between the concepts involved, (iv) frequency, prototypicality, conventionality, and familiarity of the concepts (as suggested for the degree of salience by Giora, 1997, 2003), (v) genre-knowledge, (vi) the contextual influences on the text, e.g. by semantic priming. Since there always has to be a recipient involved, some influences on the recipient's part should be considered: (i) executive function skills, (ii) pragmatic skills, (iii) degree of exposure to specific forms of verbal humor, (iv) context factors on the person (such as having heard a similar joke before or having read about cats eating birds), (v) autistic-like traits or other personality variables.

The basic idea of accessibility appears to be compatible with the "frame shifting" (Coulson, 2001) model postulated for the comprehension of jokes (Coulson \& Kutas, 1998; Coulson, 2001; Coulson \& Oakley, 2005; Coulson, Urbach, \& Kutas, 2006). The 
choice of different terminology in the present account is due to the limiting connotations of the concept "frame", originally postulated by Minsky $(1975,1977)$. A frame consists of a memorized knowledge structure, i.e. a network of semantic relationships specific for a given situation, like a "pet-frame", which includes feeding or nursing, and, on the other hand, a "predator-frame" which includes cruelty, prey, chasing, food-chain, etc. for Example (9). Shifting from one semantic field to a completely different semantic field is an essential feature of (most) GP jokes. However, in many cases (as in the following Example (10)), more than only one specific knowledge structure seems to be necessarily involved in the comprehension of discourse, such as a joke.

(10) How many mice do you need to screw in a light-bulb? Two, but the tricky part is to get them inside.

Here, the recipient has to shift from a household frame to a sexuality frame. However, it appears implausible to assume the existence of a specific frame for "mice screwing in a light-bulb" as an established concept in the memory. The construction of such a semantic representation is probably based on much richer associations than implied by the idea of a "frame". Further, not all jokes that are classified as GP jokes within the present framework involve a complete shift between frames. Examples (3) and (4) both involve the frame of physical violence among children, no matter whether the weapon is a pair of glasses or a base-ball bat. Even if one is willing to allow for a more detailed conceptualization of a frame, the important aspect of a GP joke seems to be the radical change of the semantic representation of the discourse. This change might involve frame shifting. However, it is mainly based on the revision of committed beliefs due to default reasoning processes and on the re-arrangement of the activated knowledge in interaction with the perceptual input. 
The accessibility of the hidden interpretation is, therefore, portrayed as the ease with which the relevant knowledge for the explanation of the otherwise incoherent textual input can be achieved. Once the hidden interpretation has been accessed, it has to be probabilistically integrated in the discourse comprehension structure. In Example (7), the comprehension relies on the knowledge that males sometimes feel the urge to cross-dress. Even though it is not highly common that a boy wishes to wear a bra and make-up, this knowledge seems quite easily accessible. One possible candidate for the interpretation is SU2, namely that $x$ is a boy who would love to cross-dress. The probability of the second interpretation becomes high and replaces SU1 as the highest ranked interpretation; $P(S U 2 \mid A \wedge P L)>P(S U 1 \mid A \wedge P L)$. The subsequent process of belief revision changes the semantic mental representation of the discourse. In the philosophy of science, an analogous phenomenon is called a paradigm-shift (Kuhn, 1996). Certain core aspects of a set of beliefs are consistently under strong attack. No minor adaptations of the beliefs can accommodate the new evidence, but one has to apply a completely new set of beliefs and explanations. ${ }^{3}$

\subsection{Humorous Potential}

The previous sections have avoided the most essential feature of GP jokes: they are perceived as humorous. Humor is not an inherent feature of a stimulus, such as length or weight, but it only arises as a result of dynamic interaction between some inherent structural features of a stimulus and the perception and elaboration of the stimulus by a recipient (see also Hurley et al., 2011, p.16-18). Dependent on these features, stimuli carry different degrees of potential to be perceived as humorous. With respect to the humorous potential of a GP joke, an important question is whether the GP structure

\footnotetext{
${ }^{3}$ The analogy between jokes and philosophy of science was pointed out by Ramachandran (1998).
} 
itself is already responsible for (at least a small portion) of the humor or whether the GP structure is a mere delivery mechanism for a humorous idea, or the combination of both. Incongruity models of humor (e.g., Nerhardt, 1977; Suls, 1972; McGhee, 1979; Forabosco, 1992), despite their differences in details, claim that a stimulus is perceived as humorous if it is incompatible with an expected pattern. Incongruity arises due to the comparison of a specific stimulus with its typical cognitive conceptualization as a reference. For example, the typical scenario of a bus ride involves "getting on the bus", "buying a ticket", "finding a seat", "passengers", etc. If there were a clown riding a unicorn on the bus, it would be incompatible with the cognitive pattern of the familiar scenario. This incongruity might trigger humor. Incongruity models bifurcate into two main directions, depending on the importance of the resolution of the incongruity; i.e. the necessity of finding an explanation for the presence of a clown riding a unicorn in the present example.

In a similar manner, there seems to be no agreement as to what exactly creates the incongruity in GP jokes. Very often there are different types of incongruity involved (see the discussion about the "location of the incongruity" in Ritchie, 2009, p.316-319). Applying the sequential two stages of Suls's (1972) model, one could think that the incongruity of a GP joke arises by the violation of an expectation on a communicative level, i.e. by the GP mechanism itself. The resolution of this incongruity is then achieved by finding the rule which accounts for the alternative interpretation and by arriving at the coherent reading. This incongruity resolution is supposed to be the cause of the amusement.

However, numerous, perhaps most jokes are incongruous, to a large extent, because of incompatible elements within the story, the situation, or the characters depicted in the joke. The incongruity is a "part of the conveyed scenario" (Ritchie, 2009, p.316). These incongruous elements are not compatible with the expected pattern based on the 
recipient's knowledge representation. No resolution seems to be necessary for this type of incongruity. In the mummy example, a 14-year-old boy likes to cross-dress. This idea is incompatible with the most prototypical pattern of a 14-year-old boy. Rothbart and Pien (1977) introduced the distinction between "possible" and "impossible incongruities". They pointed out that only the first kind of incongruity can be resolved completely within the joke, while the latter kind of incongruity is based on conflicts within the recipient's world knowledge and does not need a complete resolution. Attardo, Hempelmann, and Di Maio (2002) termed a comparable distinction "focal" vs. "background" incongruity. The focal incongruity is the essential mechanism of the joke, and the secondary or background incongruity enforces the humorous potential of the focal incongruity by introducing additional incongruous elements (see also Dynel, 2012, p.89). Following this distinction, one can build a dichotomy between the focal, here also called GP mechanism-based incongruity and the secondary or background incongruity due to the incongruous content of a joke. In order to disentangle these two types of incongruity, it is interesting to observe whether a transformation of the linguistic structure of a GP joke into a text without the GP mechanism could still be perceived as humorous as in (11).

(11) Peter is 14 years old. He expects to be allowed to wear a bra and make-up.

The point becomes much clearer with a transformation of joke (9) to Example (12):

(12) Susie's bird was eaten by her cat. Susie feeds her cat with birdseeds because she wants to feed her bird.

In this extreme of the dichotomy outlined, the humorous potential of the jokes is reduced to the incongruous story elements. Some of the humorous potential might remain in 
Examples (11) and (12). The focal incongruity of Example (9) was analyzed as a combination of two mechanisms. On the one hand, incongruity arises due to the crossroads mechanism of a confusing set-up. On the other hand, additional incongruity arises due to the violation of the interpretation that Susie has the intention of feeding the cat. Resolution seems necessary both for the comprehension of the joke and for the appreciation of the humor. In any case, as illustrated in (12), the representation of Susie's false logical reasoning seems to carry humorous potential independently from the underlying mechanisms of the joke. The child thinks that she can feed a bird which was eaten by the cat. This idea is incongruous compared to the recipient's world knowledge and possibly perceived as humorous on its own. But this is a question of taste and can only be tested empirically.

The other extreme of the dichotomy might be a GP sentence as Example (1). Here, it is hard to see humorous potential. The contrast between the representation of an active construction "a boat floating down the river" and the representation of a passive construction "a boat being floated down the river" might not be big enough for a humorous experience (see Dynel, 2009, p.130). More importantly, there is nothing atypical about the concept of a boat being floated down the river. There is no (secondary) background incongruity involved. GP sentences are usually not considered to carry humorous potential. If there is some humorous potential in the constructed GP joke (4), then it is probably mainly the GP mechanism which is responsible. It might not be perceived as highly humorous in the first place, but it presumably looses all humorous potential if transformed into (13).

(13) The girl was carrying a baseball-bat. The boy hit the girl.

Ritchie (2004, p.61-63) discussed the contrast, the absurdity, and the inappropriateness of the hidden interpretation as essential ingredients of incongruity-resolution accounts 
of humor in general, and of a GP joke in particular. However, one remaining question is why these descriptive features, like incongruity or absurdity, evoke humor, as put forward by Hurley et al. (2011). The probabilistic notion of knowledge representation might overcome this lack. As mentioned earlier, the human mind needs a coherent set of beliefs about its environment in order to function well, quickly, and efficiently for adaptive behavior. A prerequisite is the categorization of objects, situations, and concepts. Categorization is based on uncertainty. Categorization needs to be stable and reliable, on the one hand, but flexible and adjustable, on the other hand. Successful categorization needs to fulfill both criteria at the same time. Absurdity and incongruity could be closely connected to the lack of prototypicality. As described, incongruity arises due to surprising deviations of a stimulus in the context of a given "cognitive model of reference" (Forabosco, 1992). Absurd or incongruous entities from a cognitive psychological point of view might then be representations of highly improbable objects, situations, and events in a common environment or their interpretations, respectively. We assume that they are amusing because they enrich the probabilistic categorization. They include surprising exceptions and unusual circumstances. Within the model of Bayesian networks, they create or consolidate edges between nodes; i.e. associations and relationships between concepts and events. They alter the conditional probabilities among them and make the categorization of them more flexible. Accordingly, they help the human mind to strengthen the adaptive power of integrating surprising new input into existing categories. The focal (GP mechanism-based) incongruity, however, does not extend the probabilistic categorization. Instead, it strongly violates the expectations that are based on a specific committed interpretation. This violation demands that the recipients carry out a belief revision process, similar to what was described as a paradigm-shift. A whole new set of explanations and categorizations has to be retrieved for its resolution. Both notions of incongruity can be conducive to a humorous 
experience, but a combination of both types of incongruity might lead to a high degree of humorous potential in a GP joke.

\subsection{Empirical Assumptions and Evidence}

One of the strengths of a probabilistic account is that it allows for precise empirical predictions. Several hypotheses can be derived from the present account on GP jokes. One hypothesis is related to the probabilistic concept of salience, one to the idea of the accessibility of knowledge for the retrieval of the hidden interpretation, and one concerns the humorous potential of a GP joke. In the following, I will present these hypotheses separately and link them to empirical psycholinguistic data on GP jokes. Further, possible empirical measurements and operationalization will be discussed and outlined as ideas for potential future directions in this field of research.

The salience of the initially dominant interpretation was depicted as a function of probabilistic knowledge representations. By applying the idea of a Bayesian belief network, the source and the nature of the knowledge play a minor role. World knowledge, linguistic, pragmatic, and contextual knowledge can all be equally integrated in such a model, and the dominance of specific knowledge mainly depends on the stimulus type, i.e. the type of ambiguity which is conducive to the GP mechanism. Since the degree of salience determines the degree of the commitment to an initial interpretation, the first hypothesis can be formulated as H1.

(H1) A higher degree of salience of the initial interpretation leads to a stronger violation of the semantic coherence of a GP joke at the PL and to a more difficult semantic integration of the PL.

The degree of salience can be quantified in a rather straightforward manner for precise 
linguistic elements in terms of corpora linguistic measures. Potential variables are the frequency of a specific lexical meaning or a specific syntactic parsing structure out of context. These data are available in existing data bases. Moreover, familiarity ratings of specific linguistic elements or sentence completion tasks can provide further criteria of quantification (Giora, 2003, p.21ff). However, given the complexity and the diversity of the cues that are involved in the comprehension of a GP joke, such measures might not be sufficient. Indirect measures of the salience of a specific interpretation can be obtained from response times in a lexical decision paradigm. In such a task, participants have to decide whether a chain of letters is a correct word of a given language or not. This decision follows the presentation of the linguistic element of interest. Assuming that this linguistic element activates specific semantic networks, like gender knowledge about girls, for example, then the following word can either be related to this semantic network or not. If a word is semantically related to the semantic activation by the previously presented linguistic element, then the lexical decision is facilitated. This facilitation results in faster response times. The difference between responses to related and unrelated words, the priming effect, can be used as an indirect measurement of the salience of one specific interpretation. Such a paradigm has been conducted for the salience of ironic statements and other forms of figurative language (e.g., Giora, 2003; Giora et al., 2007), showing shorter decision times for the words which are related to the supposedly more salient interpretations like the literal meaning for irony and figurative language. The interpretation of canned jokes was investigated with a priming paradigm by Vaid, Hull, Heredia, Gerkens, and Martinez (2003). The stimuli were not explicitly classified as GP jokes, but the majority of them was based on the GP mechanism. Shorter decision times for words which were related to the initially dominant interpretation of a joke during the set-up phase were found.

Another way to measure the salience could be a task in which participants are asked to 
indicate straight away the probability of a specific interpretation. This can be carried out by analogy to the exemplary analysis of the salience of the mummy example I suggested above. In such an approach, stimuli have to be carefully analyzed in terms of the possibly engaged knowledge. An analyst needs to determine the cues and the presumably dominant and hidden interpretations. Participants could then simply rate on a scale the plausibility of the interpretations given the presented cues: e.g. "Given that a 14-year-old individual expects to wear a bra and make-up, how confident are you that this individual is female?" The ratings could then be used as a measurement for the degree of salience and can also be related to the response times in lexical decisions.

Another feasible way could consist in a manipulation of the salience of a specific interpretation by realizing a priming paradigm. Contextual priming in the present model is included in the probabilistic function of salience. Therefore, presenting a lexical prime - semantically related to the predominant interpretation - prior to the set-up of a joke should increase the salience of the initial interpretation in comparison to unrelated primes (e.g., presenting "doll" versus "table" in the mummy Example (1)).

Since hypothesis $\mathrm{H} 1$ predicts a relationship between the degree of salience and the degree of violation of expectations and the difficulty of semantic integration, reliable measures are necessary for the latter concepts. Self-paced reading time, eye-tracking and ERP data have been successfully used for the study of jokes that were described to rely on frame-shifting (Coulson \& Kutas, 1998, 2001; Coulson et al., 2006). In several studies, the authors experimentally compared two conditions: In the straight endings condition, the final word of a joke was substituted with an ending which did not violate the initially dominant interpretation. The joke ending did violate the salient interpretation and additionally needed a frame-shifting for a successful comprehension. In addition to other relevant linguistic features, the authors matched the Cloze probability of the endings between both conditions. The Cloze probability is derived from a task, in 
which participants have to complete a fragment of a text or a sentence. The number of times a specific stimulus is completed with a specific word serves as the specific Cloze probability. The findings indicated longer reading times on the final words of joke endings compared to straight endings, even though the straight endings were as unlikely to occur as the joke endings according to the Cloze probability measures. The authors interpreted these results as empirical evidence for the processing effort needed for the frame-shifting. Coulson and co-workers (Coulson \& Kutas, 2001; Coulson \& Lovett, 2004) found another interesting result. Some of the stimuli created a higher constraint on the ending of the text than other stimuli; i.e. a subset of the stimuli led to a very high Cloze probability of one specific ending. This ending was not used for the final experiment but it highlights the constraint of the co-text on the ending. Another subset of the stimuli had a low constraint on the ending of the text. We suggest, that this high vs. low constraint on the final word of the text might be related to the degree of salience. One interpretation is so dominant that most participants completed the text according to this dominant interpretation. Interestingly, there was a significant interaction between the reading times of the joke endings and the high vs. low constraint distinction of the stimuli. A similar pattern was reported for the ERP data with the same design and the same stimuli. The N400 is a negative-going ERP component, which typically increases in amplitude with the degree of expectancy violations or with the difficulty of integrating words into their semantic context, respectively (Chwilla, Brown, \& Hagoort, 1995; Kutas \& Federmeier, 2011). In the studies by Coulson and co-workers (Coulson \& Kutas, 2001; Coulson \& Lovett, 2004), this component was only affected by high-constraint jokes. These data accordingly support H1 because the constraint (possibly an indicator for the salience) interacted with two measures of the degree of incoherence (reading times and N400 component). A proper operationalization of the degree of salience, however, would shed more light on the empirical validity of H1. 
Accessibility was defined as the difficulty of the retrieval of the relevant knowledge necessary for the detection of the hidden joke interpretation. The degree of the accessibility was portrayed as a complex function. Textual features, mainly the number of necessary inferential steps and the engagement of higher-order belief representations are presumably intertwined with context influences and individual differences among readers. Based on this conceptualisation the following hypothesis (H2) can be derived and empirically challenged.

(H2) The higher the degree of the accessibility of the hidden joke interpretation, the easier is the belief revision process needed to overcome the discourse incoherence after the occurrence of PL.

Basically, this hypothesis predicts that a higher accessibility facilitates the process of joke comprehension. Again, the problem of operationalizing the degree of accessibility arises. The operationalization could be realized in several ways. A simple opportunity is a precise text-linguistic analysis of the stimuli, focussing on the inferential and cognitive effort that is needed for the comprehension. For obvious reasons, such a task is subjective and hardly feasible. A more reasonable strategy could be a manipulation of the contextual influence. As already suggested for testing H1, a priming paradigm could be realized. Here, words - again presented prior to the jokes - could prime the knowledge, which has to be involved for successful joke comprehension. For the bird seeds example, it might be sufficient to present a word like "predator" in order to increase the degree of accessibility for the comprehension of this joke. The priming should activate contents in the semantic network that ease the retrieval of the inferential step that the cat ate the bird, an essential causal enrichment for the comprehension process. A third possibility for the manipulation of the accessibility is the choice of different sample groups split by specific individual differences or by different treatments before the experiment. Ver- 
bal intelligence score, executive skills, autistic-like traits, emphatic, or pragmatic skills could be plausible factors that influence the accessibility on the participant's side.

Different background information or mood induction could also influence the ability to retrieve the necessary knowledge. For example, it has been shown that positive emotions facilitate a global cognitive style. This global cognitive style in turn facilitates tasks that rely on cognitive flexibility and mind set switching like creative problem solving. The cognitive flexibility increases the dopamine level, which in turn leads to more positive emotions (e.g., Ashby et al., 1999; Dreisbach \& Goschke, 2004). Therefore, the induction of a positive mood could facilitate the accessibility of the hidden interpretation.

Sensible indicators of the comprehension process involved could be (i) accuracy measures in comprehension tasks or comprehension ratings, (ii) response times in the comprehension tasks, (iii) number and pattern of regressive eye movements in eye-tracking reading tasks, (iv) self-paced reading times, and (v) ERP components, which are supposed to reflect working memory engagement or repair processes (e.g., the P600 component; Van Herten, Kolk, \& Chwilla, 2005). Self-paced reading times after the occurrence of PL, just as eye-tracking data, are quite problematic. Longer reading times for GP jokes might be a mixture of the violation of an expectation and the effort for a reestablishment of a coherent text. It seems hard to disentangle them in terms of how strongly the degree of salience impacted the data and how big the influence of the accessibility was. ERP data, however, suggest that the retrieval of the relevant knowledge might be reflected by a specific ERP component reported by Coulson and co-workers (Coulson \& Kutas, 2001; Coulson \& Lovett, 2004). In their study, the authors obtained an enhanced left anterior negativity between 500 and $700 \mathrm{~ms}$ after the PL onset and called it "frame-shifting component". Interestingly, this component was stronger for participants who were classified as good comprehenders. Good comprehenders simply responded more accurately to the jokes. Since the comprehension score was presented 
as a different measure for the degree of accessibility, these data do not directly support H2. But they speak for a convergent validity of the measures. The relationship between these measurements supports the idea that the ERP frame-shifting component truly reflects the cognitive processes engaged for the retrieval of the knowledge for the hidden joke interpretation. It is hypothesized that this component is triggered by a higher working memory or executive skills engagement. Accordingly, the frame shifting component could provide a reliable variable for testing $\mathrm{H} 2$.

As for the humorous potential, it seems utopian to make precise empirical predictions. Too little is still understood about the "magic" ingredients that make jokes work. This is related to the sheer complexity of different factors that have to be successfully combined for an emotional experience of humor. One question which was posed in this article was whether the humorous potential of a GP joke is based on the GP mechanism itself or whether the incongruous elements of the story or the characters involved in the joke are responsible for the amusement. Constructing stimuli on this dichotomy of focal and background incongruity might provide an option for experimental testing. Sensible indicators of emotional reactions to humor could be obtained by (i) questionnaires and rating studies, and (ii) emotion-related psychophysiological changes like, for example, changes of pupil diameter as an indicator of cognitive effort and emotional arousal (Bayer, Sommer, \& Schacht, 2011).

\subsection{Conclusion}

The phenomenon of GP jokes was described in order to illustrate the dynamic interaction between textual input and the incremental and non-monotonic meaning construction processes in the reader during discourse comprehension. In a GP joke, an initially predominant interpretation gets violated. The incoherence on the level of the mental 
representation of the discourse has to be overcome by the retrieval of a second, hidden interpretation, which re-establishes a coherent representation. Previous accounts on the phenomenon were adapted in a probabilistic way. The concept of probabilistic salience was developed in order to highlight the strong relationships between stochastic knowledge and the resolution of textual ambiguities. The probabilistic account has the advantage to model different sources of knowledge (linguistic, pragmatic, contextual, and world knowledge) in the same way, namely as conditional dependencies between concepts. Bayesian belief networks are a useful computational model for tasks which require quick and reliable decisions, on the one hand, and flexible revision processes in the light of new and unexpected evidence, on the other hand. An optimal balance of these two cognitive mechanisms was assumed as an essential element of the human mind. GP jokes seem to manifest these mechanisms on the level of communication processes. The accessibility of knowledge in the memory, which can implement the new unexpected evidence, was described as a highly complex (neuro-)cognitive function. Previous accounts have labelled this search for a coherent interpretation after the failure of the initial interpretation as frame-shifting. Despite agreeing with the basic claims of the frame-shifting idea, it was argued that the cognitive mechanism underlying this task might be much richer than it is implied by the concept of frames. Current models of the human mind, however, seem still far away from being able to handle the multi-layered, creative, associative, and multidimensional engagement of the cognitive representations of the textual environment, as they are necessary for the comprehension of jokes and verbal humor in general. Some of the ideas in this chapter are not new, but are covered within a different framework. Some new ideas for future directions in the field of discourse comprehension and humor research were presented as well, and some of these suggestions need further empirical tests. 


\section{Chapter 3}

\section{From Incoherence to Mirth}

\subsection{Introduction}

Contrary to so-called garden path sentences (Ferreira, Christianson, \& Hollingworth, 2001; Osterhout \& Holcomb, 1992), the violation and the re-analysis of GP jokes are localized at the semantic rather than syntactic level. Here, the mental representation of the discourse, theoretically depicted as mental model (Johnson-Laird, 1983) or situation model (Kintsch, 1988, 1998), is violated at the PL. It is commonly assumed that the discourse comprehension is an active process of cognitive construction that involves the integration of explicit linguistic input with other linguistic and non-linguistic context information, including new semantic and pragmatic inferences and knowledge from longterm memory. Most importantly, a committed false belief concerning the interpretation of the text has to be substituted. This "(belief) revision" of the semantic representation (of SU) is the crucial mechanism during the comprehension of GP jokes (cf. Mayerhofer \& Schacht, 2013). Consider again example (1).

(1) "Mummy, I just turned 14 years. May I please, finally, be allowed to wear a bra and make up?" -"No, and eat up your soup, my son."

Given the linguistic information and the recipient's world knowledge, the child being a girl is the most plausible interpretation of the set-up phase. This interpretation gets violated when one hears the mother calling the child "son" (PL), thus leading to 
incongruity. Belief revision occurs, and the recipient represents a boy who would love to wear a bra and make up. This incongruity resolution, in combination with the activation of the alternative, hidden interpretation and with its "inappropriateness" (Ritchie, 2004, p.61), is typically accompanied by the experience of laughter and mirth in the recipient.

Many researchers agree upon the outlined sequential comprehension process, supported by empirical evidence. Vaid et al. (2003) demonstrated priming effects due to the dominant semantic networks specifically activated at different stages of joke comprehension over time. Coulson and Kutas (1998) found longer reading times for joke endings compared to straight (coherent) endings. These longer reading times were also accompanied by regressive eye movements after reading of the punch-line (Coulson et al., 2006). Evidence for the enhanced costs of semantic revision also comes from non-joke texts (Carreiras, 1996; Sturt, 2007).

Recently, several studies using event-related brain potentials (ERPs) have investigated the processing of jokes and verbal humor. Three (groups of) ERP components were especially fruitful for the study of verbal humor: the N400, late positivities, and the left anterior negativity. The N400 component (Kutas \& Hillyard, 1980) is an enhanced negative-going deflection at cento-parietal electrodes starting around 200-250 ms after stimulus onset and lasting until 500-550 ms after stimulus onset with a peak around $400 \mathrm{~ms}$, hence the name. It reliably occurs with semantic violations during sentence or discourse comprehension (Berkum, Hagoort, \& Brown, 1999). Other important factors that influence the amplitude of the N400 component are the predictability of a word in a given context, as for example reflected by the Cloze-probability (Kutas \& Hillyard, 1984), and the semantic relatedness between the context and the expected word. The N400 effect functionally reflects semantic integration difficulties at the interface of word/stimulus recognition, linguistic and nonlinguistic context, and conceptual binding with the long-term-memory during an active comprehension process (Kutas \& 
Federmeier, 2011). Previous ERP studies on joke comprehension have led to heterogeneous evidence regarding N400 effects. Derks, Gillikin, Bartolome-Rull, and Bogart (1997) found augmented N400 amplitudes for jokes that also elicited a higher activation of the zygomatic muscle, indicating the elicitation of positive emotions. Coulson and Kutas (2001) found an N400 effect for joke endings involving frame shifting compared to straight endings. The effect was restricted to jokes with high semantic constraint on the ending. This finding was replicated in follow-up studies, shown to be only present for participants with a low verbal intelligence score (Coulson \& Lovett, 2004), and to be related to the visual field of the stimulus presentation (Coulson \& Williams, 2005).

Several ERP studies on language comprehension demonstrated syntactic violations to elicit an augmented positivity at posterior scalp sites. This so-called P600 component usually starts around $600 \mathrm{~ms}$ after stimulus onset and lasts until around $1200 \mathrm{~ms}$. Since these late positivities are especially triggered by syntactic anomalies, such as in GP sentences (Bever, 1970; Osterhout \& Holcomb, 1992), they are commonly considered to reflect syntactic repair processes which occur after the detection of a syntactic violation for the initially dominant parsing. However, Van Herten et al. (2005) found posterior P600 effects for semantic anomalies, and experimentally ruled out the possibility of a hidden syntactic anomaly being responsible for the component. This finding led the authors to argue that the P600 is a form of monitoring component "that checks upon the veridicality of one's analysis" (Van Herten et al., 2005, p.254). In line with this assumption, the P600 has been suggested to reflect a combinatorial process, integrating both syntactic and semantic features of a sentence (e.g., Wicha, Moreno, \& Kutas, 2004; Martín-Loeches et al., 2009), and has also been reported for increased discourse complexity (Burkhardt, 2007). Moreover, a late positivity effect - distinguishable from the typical P600 effect by its frontal distribution - has been reported (Schacht et al., 2010) and related to the complexity and the ambiguity of a text (Kaan \& Swaab, 2003). 
In many regards, GP jokes might be assumed as a semantic equivalent of GP sentences. Thus, the question is whether a semantic repair process in jokes - such as the belief revision - triggers similar brain response patterns as the mainly syntactic repair processes (P600 at posterior sites). Previous evidence has partly indicated such similarity, but remains incomplete (Coulson \& Lovett, 2004; Marinkovic et al., 2011).

Apart from the P600 like findings, there is strong evidence that joke endings, triggering believe-revision processes, elicit a left-lateralized sustained anterior negativity (Late Left Anterior Negativity; LLAN), between 500 and $900 \mathrm{~ms}$ after stimulus onset. This component has been shown only for good comprehenders (Coulson \& Kutas, 2001; Coulson \& Williams, 2005) or restricted to left-handed participants (Coulson \& Lovett, 2004). Coulson and co-workers suggested that the component reflects the successful comprehension of jokes and called this effect "frame-shifting component" according to their terminology. The LLAN has also been considered to reflect working memory activity necessary for the computation of a new mental representation of the discourse (Baggio, Van Lambalgen, \& Hagoort, 2008; Meltzer \& Braun, 2013; Münte, Schiltz, \& Kutas, 1998).

GP jokes also reliably lead to the subjective experience of mirth. Therefore, one might expect other ERP components elicited by jokes, reflecting the emotional processes. Emotion-related ERP responses to humorous visual stimuli have been reported as Posterior Positivities between 300 and 600 ms after the onset (Gierych, Milner, \& Michalski, 2005; Korb, Grandjean, Samson, Delplanque, \& Scherer, 2012). These components show strong similarities to the late positive complex (LPC), which has repeatedly been shown in response to emotional stimuli, such as affective pictures (e.g., Cuthbert, Schupp, Bradley, Birbaumer, \& Lang, 2000; Schupp et al., 2000), and to facial emotional expressions and emotional words (e.g., Schacht \& Sommer, 2009a, 2009b). This effect has been related to sustained, elaborative processing of emotional relevance of a given stim- 
ulus. At longer latencies, Du et al. (2013) reported an enhanced positivity to Chinese jokes compared with neutral Chinese texts between 1250 and $1400 \mathrm{~ms}$ after the stimulus onset, which the authors related to an affective stage of the joke processing.

It is the main aim of the present study to disentangle different sub-processes or processing stages, respectively, involved in the comprehension of GP jokes to be reflected in distinguishable ERP components over time. At least, three different processing stages are hypothesized to be involved: (a) the violation of the pre-dominant initial semantic representation, (b) the revision of this semantic representation, and (c) the occurrence of an emotional reaction. To this aim, we constructed parallel versions of selected jokes in such a way that all comprehension processes should remain constant apart from the processes of interest outlined above. This manipulation was realized by exchanging only the final word of the original jokes as in the following examples (compared to (1)):

(2) "Mummy, I just turned 14 years. May I please, finally, be allowed to wear a bra and make up?" -"No, and eat up your soup, my girl."

(3) "Mummy, I just turned 14 years. May I please, finally, be allowed to wear a bra and make up?" -"No, and eat up your soup, my father."

In example (2), the interpretation of the whole discourse is straight-forward and coherent. Thus, no belief revision is necessary. In example (3), the initial interpretation gets violated. The final sentence is a grammatically and semantically correct sentence, but its final word is discourse incoherent, thus triggering revision processes. In contrast to the joke ending of example (1), no hidden interpretation (or at least no plausible one) can be activated and no alternative meaningful coherent representation of the text can be constructed. This makes the whole text incomprehensible. The joke endings share the discourse incoherence with (3) at the occurrence of the final word (PL), but it shares the comprehensibility of a meaningful discourse with (2), once the belief re- 
vision has been successfully carried out. In a series of experiments, we investigated the neuro-cognitive processes being specific for GP jokes, using 48 GP jokes and their coherent and incoherent manipulations as stimuli. Experiment 1 focused on behavioral measures using a self-paced reading time paradigm. Here, I expected increasing reading times from coherent over incoherent to joke endings. In Experiment 2 to 4, ERPs were of main interest in order to localize the GP-specific sub-processes. Hypotheses were as follows: Joke endings and incoherent endings both represent the violation of the initially dominant semantic representation and should thus elicit an augmented N400 component. Successful belief-revision processes in GP joke comprehension - requiring enhanced inferential and working-memory related processes - should be reflected in the occurrence of LLAN components. Only joke endings should elicit an emotional response. Therefore, we expected emotion-related ERP components at subsequent, late stages of joke processing, namely following the violation and the revision processes.

Another potentially fruitful indicator of both cognitive and emotional processes during the comprehension of jokes could be provided by pupillary responses, which we also measured in Experiment 2. First, changes of pupil size have been shown to be a sensitive measure for the cognitive load during a task: Higher cognitive load leads to larger pupil dilation (Kahneman \& Beatty, 1966; Van Der Meer et al., 2010). Second, larger pupil dilations have also been reported in association with higher emotional involvement, related to the arousal (Bradley, Miccoli, Escrig, \& Lang, 2008) or to the intensity (Partala \& Surakka, 2003) of an emotional reaction. Both factors cognitive load and emotional processing have been shown to affect pupil dilations also in the processing of verbal stimuli, such as single word processing and recognition (Bayer et al., 2011; Võ et al., 2008). Since the successful comprehension of GP jokes is hypothesized to involve both increased cognitive processing effort and an emotional response, we expected larger pupil dilations after joke endings compared to coherent endings. Changes of pupil 
size to incoherent endings should be intermediate due to enhanced cognitive demands (violation detection) on the one hand but the absence of both revision processes and emotional response on the other hand.

\subsection{Experiment 1: Reading Times}

The comprehension process of GP jokes is considered to contain two important stages: the detection of the violation of the semantic representation and the belief revision process. Both factors should lead to enhanced cognitive load which should be reflected in an increase of the reading times at the final word, the PL, compared to coherent endings, as previously shown for English material (Coulson \& Kutas, 1998). In the present experiment, we expected similar results for our German stimuli. Moreover, we were interested in differences between the reading times of joke compared to incoherent endings. This comparison aimed at investigating at which point of the discourse comprehension process readers realize whether it makes sense to search for a hidden coherent interpretation. If participants understand at a very early stage after the onset of the final word that there will not be a hidden joke interpretation, this should be reflected by shorter reading times for the incoherent endings compared to the joke endings. This would indicate that parallel to the violation of the semantic representation at the joke ending readers get some sort of hinge that it makes sense to pursue a re-interpretation and belief revision for the joke endings. This hinge should be absent for incoherent endings. If this is not the case joke endings and incoherent endings should trigger the same cognitive attempt to find a coherent meaning construction. Another question was, how long a participant will search for a new coherence until she or he realizes that this search is in vain in the case of an incoherent ending. Longer reading times for incoherent endings compared to joke endings would thus indicate a very persistent willingness to 
re-establish a coherent interpretation for an incoherent text.

\subsubsection{Method}

\section{Participants}

Twenty-four participants (16 females), ranging in age between 18 and 29 years $(M=$ $22.48, S D=2.93)$, were tested. All of them were native speakers of German and students at the University of Göttingen, coming from a wide range of disciplines. They were rewarded with $€ 8 / \mathrm{h}$ for their participation.

\section{Material}

A total number of 144 stimuli was constructed. Forty-eight jokes were selected from different sources according to the following criteria: (i) They had to exploit the GP mechanism. Additionally, they were selected to be (ii) ethically acceptable, (iii) subjectively amusing, (iv) translatable into German, unless they were originally German, without losing the amusement potential and without destroying the underlying GP structure, and (v) rewritable in such a way that the very final word of the last sentence could serve as the crucial PL element. Based on these 48 jokes, two additional versions were constructed by exchanging only the final word of the text. In the Coherent condition, the final word of the joke was replaced by a word which was coherent according to the initial first interpretation of the text. In the Incoherent condition, the final word was replaced by a word which is incoherent according to the first interpretation and which does not offer a hidden interpretation of the set-up. Importantly, this final word violated neither the syntactic nor the semantic structure of the last sentence but it did not fit into the whole discourse of the text. This led to a total number of 144 stimuli with 48 text fragments identical in all three conditions 
Table 3.1: Descriptive data of the matched stimulus features.

\begin{tabular}{|c|c|c|}
\hline Variable & $M$ & $S D$ \\
\hline \multicolumn{3}{|c|}{ (a) Coherent } \\
\hline Number of Letters & 8.17 & 2.9 \\
\hline Frequenc: & 12.62 & 3.62 \\
\hline \multicolumn{3}{|c|}{ (b) Incoherent } \\
\hline Number of Letters & 7.9 & 3.13 \\
\hline \multicolumn{3}{|l|}{ (c) Joke } \\
\hline er of Letters & 7.81 & 2.94 \\
\hline Word Frequency & 11.88 & 3.9 \\
\hline
\end{tabular}

but varying final words between conditions. Final words were matched between conditions according to Word Category, Word Frequency (Leipziger Worthäufigkeitsklasse; http://wortschatz.informatik.uni-leipzig.de/), and Word Length (number of letters). Descriptive statistics of the material is reported in Table 3.1.

In pre-experimental ratings, 68 participants (46 females) between 18 and 36 years $(M=23.19, S D=3.38$ ) evaluated on 5 -step scales from 1 (trifft überhaupt nicht zu/do not agree at all) to 5 (trifft völlig zu/ totally agree). Items were constructed according to three theoretically derived dimensions: humorous potential (Humor), predictability of the ending (Predictability), and comprehensibility of the whole text (Comprehensibility). For each dimension, three items were constructed in order to obtain: (i) a behavioral component, (ii) a cognitive appraisal, (iii) an emotional response (see Appendix A for all items). These three items per scale were summed together for the three total scale scores. The results of the ratings are depicted in Figure 3.1.

ANOVAs and Bonferroni-corrected post-hoc t-tests were carried out for the three scales. Only texts that were indicated as unfamiliar to the participants were included in the analysis. There was a significant effect of Condition on all three scales: Humor, $F(2,141)=$ 135.31, $p<.001$, Predictability, $F(2,141)=77.48, p<.001$, and Comprehensibility, 
(a) Humor potential

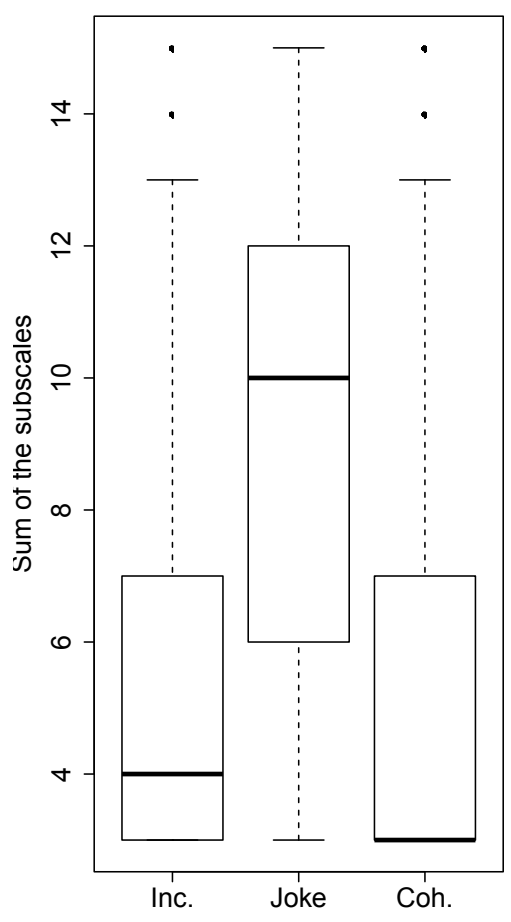

(b) Predictability

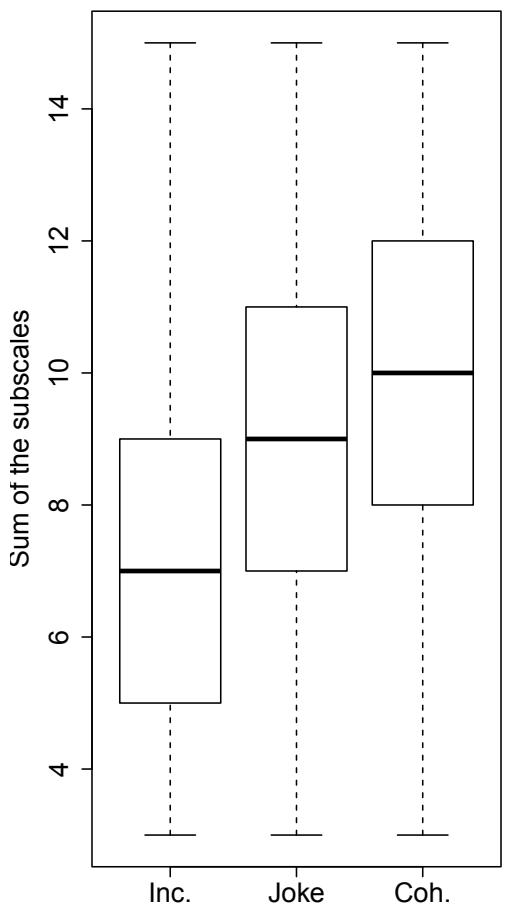

(c) Comprehensibility

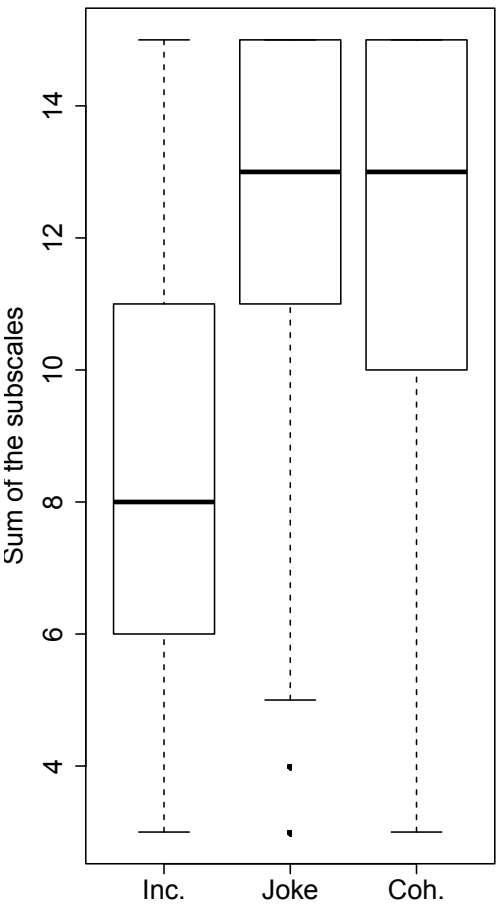

Figure 3.1: Box plot of the three scales of the ratings. Every data point is one observation of one participant and one stimulus. The thick line is the median, the box represents the $25 \%$ and $75 \%$ quantiles, and the whiskers are the minimum and maximum values, while points represent statistical outliers.

$F(2,141)=115.45, p<.001$. The Joke condition $(M=8.83, S D=1.04)$ was rated as more humorous than both the Coherent $(M=5.39, S D=1.42), t(94)=14.52, p<$ .001 , and the Incoherent condition $(M=5.5, S D=1.06), t(94)=13.29, p<.001$, while there was no significant difference between Coherent and Incoherent. The Joke condition $(M=8.83, S D=1.04)$ was rated less predictable than Coherent $(M=$ $9.92, S D=1.24), t(94)=-4.66, p<.001$, but more predictable than Incoherent $(M=7.07, S D=1.11), t(94)=8.04, p<.001$. Thirdly, the Incoherent condition $(M=8.33, S D=1.68)$ was rated less comprehensible than the Joke condition $(M=12.25, S D=1.12), t(94)=-13.41, p<.001$, and than the Coherent condition $(M=11.89, S D=1.32), t(94)=-11.51, p<.001$, while there was no significant differ- 
ence between Joke and Coherent. Ratings confirmed the validity and the suitability of the stimulus material. The 144 stimuli (48 Joke, 48 Coherent, 48 Incoherent) were used for Experiment 1. In addition, 144 Filler items were constructed as similar as possible to the original stimuli in terms of the linguistic style, e.g., syntactic structure, topic, lexical level, dialogs, etc. Similar to the experimental stimuli, identical 48 text fragments were completed with three different endings: two different coherent endings and a discourse-incoherent ending. The filler items fulfilled the following functions: (i) They diluted the proportion of jokes, supposed to make the purpose of the study less obvious. (ii) They reduced the proportion of repetitions of the text fragments and, should, therefore, distract the participants from keeping all the text fragments in memory. Note that responses to fillers were not analyzed. The total of 288 texts was distributed to three different sets (every set containing 96 different text fragments). The order of the texts within a set was randomized for every participant and the six possible permutations of the block order were equally distributed over all the participants, resulting in 288 short texts of six conditions (Joke, Coherent, Incoherent, Filler 1, Filler 2, Filler Incoherent) for each participant. This guaranteed that possible influences by the repetition of the text fragments were at least equally balanced across the participants and the conditions.

\section{Procedure}

The experiment was carried out in a group lab on a computer with four participants per session. After they had indicated the demographic data, participants received instructions on the computer screen that they participated in an experiment on text comprehension. They were made familiar with the presentation of the stimuli and were told to carefully read the texts. They were explicitly told that some of the texts were hard to understand, and that some of them did not make sense at all. Also, they were explicitly instructed to continue with the next stimulus when they think that they understood 
the text or when they are sure that the text does not make sense.

The texts were presented on a computer screen with an adapted version of the Moving Windows Paradigm (Just, Carpenter, \& Woolley, 1982), implemented by Pygame, a graphical interface for Python. In a first step, the whole text was presented to the participant with the final sentence of the text being masked by blanks. The last sentence of the text appeared word by word after the participants pressed the return key on a standard keyboard. Only the actual word appeared unmasked, and the words that had been read became masked again. Most importantly, the reading time for the final word (the crucial manipulation of the experiment) was measured as the time between the onset of the final word and the moment a participant pressed the return key on the keyboard in order to proceed with the next text.

After a pseudo-randomly chosen amount of trials (normal distribution with $M=10, S D=$ 4), participants were presented with a statement concerning the previously presented text and had to indicate whether the statement was true in relationship to the currently read text. The comprehension question was randomly chosen to aim for a correct "true" or a correct "false" answer. The comprehension task had the main function of preventing participants from clicking themselves through the task without proper processing of the stimuli.

\section{Results}

Responses below $200 \mathrm{~ms}$ and above 3 standard deviations above participant's average were excluded from the analysis. Every participant's mean reading times of the final word per condition (Incoherent, Joke, and Coherent) were calculated and logtransformed. A one-way ANOVA revealed a significant main effect of condition, $F(2,46)=$ $8.51, p<.001, \eta_{G}^{2}=.27$, with significantly shorter reading times for coherent $(M=$ 
$1018, S D=329)$ as compared to joke endings $(M=1162, S D=446), t(23)=$ $-3.97, p<.001, d=-1.29$, and to incoherent endings $(M=1111, S D=403), t(23)=$ $-2.62, p=.015, d=-.91$. The latter did not differ significantly, $t(23)=1.49, p=$ $.149, d=-.43$.

\subsubsection{Discussion}

The hypothesis of longer reading times for joke endings compared to coherent endings was clearly supported by the data. Further, the reading times of the joke endings tended to be prolonged in comparison to incoherent endings, but this difference failed significance. Reading of incoherent endings took also significantly longer than of coherent endings. Together, these findings indicated that either the detection of the semantic incoherence itself is characterized by higher processing demands or the participants started the same attempt of finding an alternative interpretation as in the joke endings, possibly, triggered by the occurrence of jokes during the experiment.

\subsection{Experiment 2: Evidence from ERPs and changes of pupil size}

Reading times, as measured in Experiment 1, reflect only the sum of several subprocesses, thus not allowing any specific assumptions regarding specific processing stages. ERPs provide the advantage of high temporal resolution in the range of milliseconds. Therefore, distinguishable ERP components can be related more precisely to the hypothesized underlying cognitive or emotional processing stages involved. Here, we recorded ERPs and the changes of the pupil size in relation to the different endings of the stimulus material in order to investigate the hypothesized comprehension processes as outlined 
in the introduction.

\subsubsection{Method}

\section{Participants}

Twenty-five students from different disciplines participated in this experiment. All of them were native speakers of German. From this sample, data of four participants had to be removed from analysis because they were familiar with too many of the jokes $(N=2)$ or because of excessive number of EEG artifacts $(N=2)$. The remaining 21 participants (14 females) were between 20 and 34 years old $(M=24.2, S D=3.82$ ) and had an LQ score between -90 and $100(M=60.4, S D=57.34)$, according to the Edinburgh Handedness Inventory (Oldfield, 1971). All reported normal or correctedto-normal vision and no neurological or neuropsychological disorders. Participants gave their informed consent and received $€ 20$ or course credit. None of them had participated in the rating experiment or in Experiment 1.

\section{Material}

Exactly the same stimulus material was used as in Experiment 1.

\section{Procedure}

Participants were seated in a dimly illuminated, sound-attenuated, and electrically shielded chamber, facing a monitor at a distance of $60 \mathrm{~cm}$. They were made familiar with the presentation of the stimuli and were instructed to carefully read the texts. They were explicitly told that some of the texts are hard to understand and that some of them do not make sense at all. 
The texts were presented on the computer screen with an adapted version of an RSVP (rapid serial visual presentation) paradigm, implemented by Pygame in black on lightyellow background. Each trial consisted of the following sequence: The text fragment (without the final sentence) was presented at the center of the screen. After a button press, the final sentences began with a fixation cross of $500 \mathrm{~ms}$ duration and was presented word-by-word, with $250 \mathrm{~ms}$ duration per word and $500 \mathrm{~ms}$ SOA. After the critical final word disappeared, another fixation cross was presented for $5000 \mathrm{~ms}$, which was followed by a comprehension task. A statement concerning the preceding text was presented and participants were asked to indicate whether the statement is correct or not by pressing one of two buttons. The questions were pseudo-randomly chosen to be either correctly accepted or declined. Afterwards, participants had to indicate on a questionnaire which of the jokes they were sure that they had been familiar with prior the experiment.

\section{Psychophysiological recordings, processing, and analysis}

ERPs were recorded from 68 active $\mathrm{Ag} / \mathrm{AgCl}$ electrodes located according to the extended 10-20 system (Pivik et al., 1993). Sixty-four electrodes were placed in an electrode cap. External electrodes were used for the vertical and horizontal electrooculogram (left eye) and left and right mastoid. EEG signals were amplified by a Biosemi ActiveTwo AD-box, referenced to the common mode sense (CMS; active electrode) and grounded to the driven right leg (DRL; passive electrode). All channels were recorded with a passband of $0.16-100 \mathrm{~Hz}$; sampling rate was $512 \mathrm{~Hz}$. Offline, the continuous EEG record was converted to average reference, corrected for blinks using Surrogate Multiple Source Eye Correction (MSEC; Ille, Berg, \& Scherg, 2002) as implemented in BESA (Brain Electric Source Analysis, MEGIS Software GmbH) and filtered with a $30 \mathrm{~Hz}$ low-pass filter. Continuous EEG data were segmented into epochs of $1200 \mathrm{~ms}$, starting 
$200 \mathrm{~ms}$ before the onset of the critical (final) word. All ERPs were referred to a $200 \mathrm{~ms}$ pre-stimulus baseline. Epochs containing artifacts were automatically discarded when any amplitude exceeded -100 or $+100 \mu \mathrm{V}$ or when any voltage step exceeded $50 \mu \mathrm{V}$ per sampling point in any of the channels. Data of two participants were dropped from analyses (less than $50 \%$ of the trials remained). For all remaining participants, between $61 \%$ and $100 \%$ of the trials $(M=89.94, S D=10.82)$ entered the analysis, with the additional criteria of correct responses to comprehension questions and indicated unfamiliarity of jokes. In total, 20 to $37(M=29.1, S D=5.8)$ trials per participant, electrode, and experimental condition were averaged. All ERPs were referred to a 200 ms pre-stimulus baseline.

Based on the literature and on visual inspection of the data, mean ERP amplitudes were calculated in the three following time windows after stimulus onset: $250-500 \mathrm{~ms}$ (N400), 500-700 ms (LLAN), and 700-1000 ms (late posterior positivities) and submitted to repeated measures overall ANOVAs including the factors Condition (Coherent, Incoherent, Joke) and Electrode (66). By definition, the average reference sets the mean value of the ERP amplitude to zero across all electrodes within a given condition. Therefore, for these ANOVAs, only effects in interaction with electrodes are meaningful. In addition to analyses including all electrodes, effects were assessed by ANOVAs on ERPs on relevant electrode groups defined by visual inspection of difference maps (region-of-interest analysis, ROI). Between 250 and $500 \mathrm{~ms}$ (N400), ERP amplitudes were assessed at a group of central electrodes (FC1, FCz, FC2, C1, Cz, C2, CP1, CPz, CP2). ERPs in the subsequent time window (500 to $700 \mathrm{~ms}$, LLAN) were assessed at left anterior electrodes (AF7, AF3, F7, F5, FT7, FC5, T7, FP1); between 700 and 1000 ms (late positivities), ERP amplitudes were quantified at a group of frontal electrodes (FP2, AF8, F8, F6, F4, AF4, Fz, F2, F1, AFz, FPz). These ROI analyses included the factors Electrode (9, 8, or 11, respectively) and Condition (3). 
In all analyses, Huynh-Feldt correction was applied to adjust the degrees of freedom of the F-ratios. Please note that all within-subject repeated ANOVA measures will be reported with uncorrected degrees of freedom but Huynh-Feldt corrected p-values. In all cases, for post-hoc pair-wise comparisons alpha levels were Bonferroni-corrected.

Pupil sizes were continuously tracked with a Desktop Mount Eye-tracking System, EYELINK 2000 by SR Research. The method was elliptic tracking of the dominant eye at a $50 \%$ illumination rate and a $1000 \mathrm{~Hz}$ sampling rate. The head position was stabilized with a chin and a forehead rest. Each block of the experiment was started with a 9-point-calibration and validation phase of the eye tracking. Offline, continuous data were segmented into epochs of $3200 \mathrm{~ms}$, starting $200 \mathrm{~ms}$ before the onset of the final word; segments were referred to the $200 \mathrm{~ms}$ pre-stimulus interval. Incorrectly answered trials and trials with jokes that were familiar to the participants before the experiment were removed. Trials with blinks were removed; the missing data were interpolated with the preceding and following 50 samples. ANOVAs with Condition as a withinfactor (three levels) were conducted on averaged data in consecutive $50 \mathrm{~ms}$ segments in order to detect the onsets of significant differences. Bonferroni-corrected post-hoc t-tests for paired samples were further applied in case of significant main effects. The data from participants excluded from ERP analysis did not enter these analyses.

\subsubsection{Results}

\section{Behavioral Data}

The test scores (together with the demographic data) are presented in Table 3.2. ANOVA on Comprehension scores showed a significant effect of Condition, $F(2,40)=29.58, p<$ $.001, \eta_{p}^{2}=.59$, with significantly more correctly answered trials in the Coherent than in both the Joke, $t(20)=6.59, p<.001, d=-1.52$, and in the Incoherent condition, 
Table 3.2: Descriptive data of the behavioral variables in Experiment $2(N=21)$

\begin{tabular}{lcc}
\hline \hline Variable & $M$ & $S D$ \\
\hline Comprehension Total & 85.52 & 3.33 \\
Comprehension Coherent & 88.59 & 3.99 \\
Comprehension Incoherent & 77.78 & 5.94 \\
Comprehension Joke & 76.19 & 8.03 \\
Number of Familiar Jokes & 7.9 & 6.38 \\
\hline \hline
\end{tabular}

$t(20)=8.13, p<.001, d=-1.8$. There was no difference in the comprehension accuracy between the Joke and the Incoherent condition, $t(20)=-0.82, p=.430, d=-.17$.

\section{Electrophysiological Data}

N400 N400. As can be seen in Figure 3.2 (first panel), an enhanced negativity over the vertex occurred, showing the typical distribution of the N400 component. The overall ANOVA of mean ERP amplitudes between 250 and $500 \mathrm{~ms}$ revealed a significant Condition by Electrode interaction, $F(130,2600)=3.71, p<.001, \epsilon=.144, \eta_{p}^{2}=.156$, reflecting larger N400 amplitudes for incoherent, $F(65,1300)=5.93, p<.001, \epsilon=$ $.144, \eta_{p}^{2}=.229$, and joke endings, $F(65,1300)=2.46, p=.004, \epsilon=.191, \eta_{p}^{2}=.109$, compared to coherent endings. Further, incoherent endings elicited larger amplitudes compared with joke endings, $F(65,1300)=2.55, p=.002, \epsilon=.217, \eta_{p}^{2}=.113$. Results of overall ANOVAs were verified by the ROI analysis showing a significant main effect of Condition, $F(2,40)=15.58, p<.001, \epsilon=1, \eta_{p}^{2}=.438$, but no significant interaction between Condition and Electrode, $F(16,320)=1.25, p=.256, \epsilon=.641, \eta_{p}^{2}=.059$. Again, post-hoc comparisons revealed significant differences between all three conditions: Joke vs. Coherent, $F(1,20)=16.7, p=.001, \epsilon=1, \eta_{p}^{2}=.455$, Incoherent vs. Coherent, $F(1,20)=19.79, p<.001, \epsilon=1, \eta_{p}^{2}=.497$, Incoherent vs. Joke, $F(1,20)=8.17, p=.010, \epsilon=1, \eta_{p}^{2}=.290$. 
LLAN/sustained N400 In the following interval from 500 to $700 \mathrm{~ms}$, the overall ANOVA of mean ERP amplitudes revealed a significant interaction between Condition and Electrode, $F(130,2600)=2.56, p<.001, \epsilon=.169, \eta_{p}^{2}=.114$. Post-hoc comparisons revealed significant differences between Incoherent and Coherent, $F(65,1300)=$ $3.12, p<.001, \epsilon=.200, \eta_{p}^{2}=.135$, and between Incoherent and Joke, $F(65,1300)=$ $3.13, p=.001, \epsilon=.169, \eta_{p}^{2}=.135$, whereas the conditions Joke and Coherent did not statistically differ, $F(65,1300)=1$.

The ROI analysis of LLAN electrodes showed a significant main effect of Condition, $F(2,40)=8.76, p=.001, \epsilon=1, \eta_{p}^{2}=.305$, and no significant interaction between Condition and Electrode, $F(14,280)=1.33, p=.263, \epsilon=.312, \eta_{p}^{2}=.062$, reflecting larger amplitudes to incoherent compared to both coherent, $F(1,20)=12.19, p=.002, \epsilon=$ $1, \eta_{p}^{2}=.379$, and joke endings, $F(1,20)=11.61, p=.003, \epsilon=1, \eta_{p}^{2}=.367$, while ERPs to coherent and joke endings did not differ from each other, $F(1,20)<1$.

However, as can be seen in the depicted maps of ERP differences of Figure 2, incoherent endings and also joke endings - even though to a smaller extent - elicited an N400like component rather than a LLAN modulation within this interval. Therefore, we carried out an additional ROI analysis, selecting a group of central electrodes (FC1, $\mathrm{FCz}, \mathrm{FC} 2, \mathrm{C} 1, \mathrm{Cz}, \mathrm{C} 2, \mathrm{CP} 1, \mathrm{CPz}, \mathrm{CP} 2)$, resembling the maximum of this sustained N400 effect. This analysis revealed a significant main effect of Condition, $F(2,40)=$ $8.04, p=.002, \epsilon=1, \eta_{p}^{2}=.287$, and a significant Condition by Electrode interaction, $F(16,320)=1.97, p=.036, \epsilon=.651, \eta_{p}^{2}=.09$, indicating slight topographical shifts between conditions. Both incoherent and joke endings elicited larger negativities in comparison to coherent endings, $F(1,20)=12.99, p=.002, \epsilon=1, \eta_{p}^{2}=.394$, and, $F(1,20)=7.91, p=.011, \epsilon=1, \eta_{p}^{2}=.283$, respectively, whereas no significant difference occurred between ERPs elicited by incoherent compared to joke endings, $F(1,20)=$ $3.24, p=.087, \epsilon=1, \eta_{p}^{2}=.140$. 
Late Positivities The overall ANOVA of mean ERP amplitudes between 700 and $1000 \mathrm{~ms}$ revealed a significant interaction between Condition and Electrode, $F(130,2600)=$ $2.27, p=.001, \epsilon=.17, \eta_{p}^{2}=.102$, reflecting enhanced anterior positivities (see Figure 2) to joke endings in comparison to both coherent, $F(65,1300)=3.06, p<.001, \epsilon=$ $.228, \eta_{p}^{2}=.133$, and incoherent endings, $F(65,1300)=2.17, p=.006, \epsilon=.246, \eta_{p}^{2}=$ .098, whereas no significant difference between ERPs to incoherent versus coherent endings occurred, $F(65,1300)=1.77, p=.051, \epsilon=.191, \eta_{p}^{2}=.081$.

As in the overall ANOVA, analyses of mean ERPs at selected anterior electrodes revealed a significant main effect of Condition, $F(2,40)=9.06, p=.001, \epsilon=1, \eta_{p}^{2}=.312$, but no significant Condition by Electrode interaction, $F(20,400)=1$. Again, in post-hoc comparisons significant ERP differences between joke and coherent endings, $F(1,20)=$ $16.71, p=.001, \epsilon=1, \eta_{p}^{2}=.455$, and joke and incoherent endings, $F(1,20)=12.78, p=$ $.002, \epsilon=1, \eta_{p}^{2}=.390$, have been found, whereas ERPs to coherent and incoherent endings not significantly differed, $F(1,20)=0$.

\section{Pupil Size data}

The ANOVAs on mean pupil sizes in consecutive $50 \mathrm{~ms}$ time windows, including the factor Condition, revealed significant differences between 800 and $3000 \mathrm{~ms}, F s(2,40)=$ 4.304 to $30.15, p s<.05$ to $<.001, \eta_{p}^{2}=.16$ to .6 . Bonferroni-corrected t-tests indicated significantly larger pupil sizes in response to Joke compared to Incoherent and Coherent after $850 \mathrm{~ms}$. This effect lasted until the end of the segmentation, $\operatorname{ts}(23)=2.57$ to $6.27, p s<.05$ to $<.001, d s=.52$ to 1.36 . Further, Coherent elicited significantly larger pupil sizes than Incoherent, starting at $2000 \mathrm{~ms}$ lasting until the end of the segmentation, $t_{\mathrm{s}}(23)=-2.55$ to $3.03, p \mathrm{~s}<.05, d \mathrm{~s}=-.52$ to -.65 (see Figure 3.3). 




C5



P5

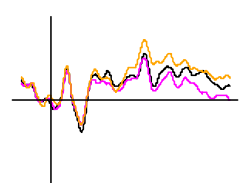

AFz

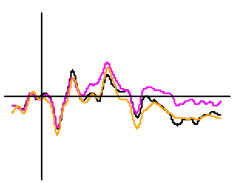

$\mathrm{Cz}$

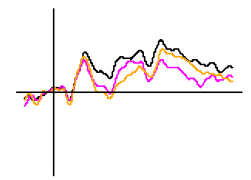

Pz

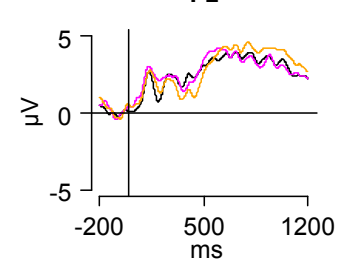

- Coherent

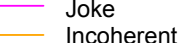

AF8

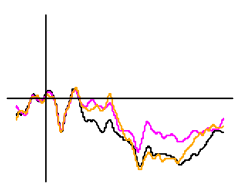

C6

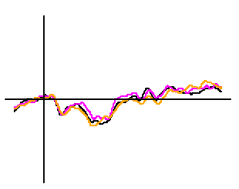

P6

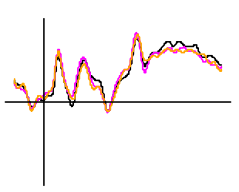

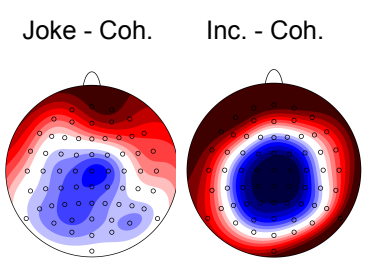

$250 \mathrm{~ms}-500 \mathrm{~ms}$

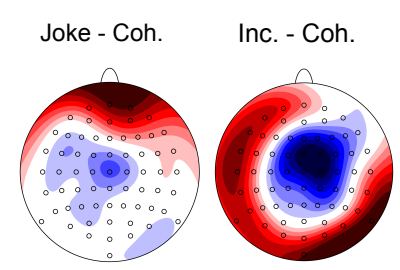

$500 \mathrm{~ms}-700 \mathrm{~ms}$

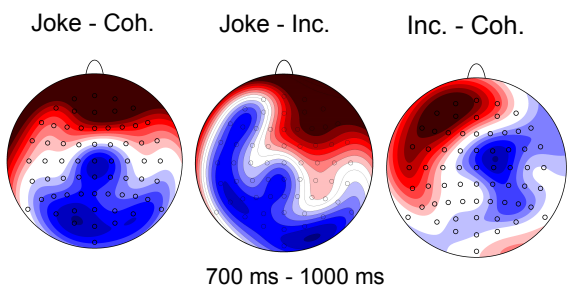

$-1.5 \mu \mathrm{V} \quad 0 \mu \mathrm{V}-1.5 \mu \mathrm{V}$

Figure 3.2: Difference maps of the conditions for the three time windows and grand average waves for selected electrodes as a function of Time relative to the stimulus onset and Condition in Experiment 2.

\subsubsection{Discussion}

As hypothesized and in line with previous findings (Coulson \& Kutas, 2001; Derks et al., 1997), joke endings elicited more negative ERP amplitudes at central and centroparietal electrode sites compared to the Coherent condition between 250 and $500 \mathrm{~ms}$ 


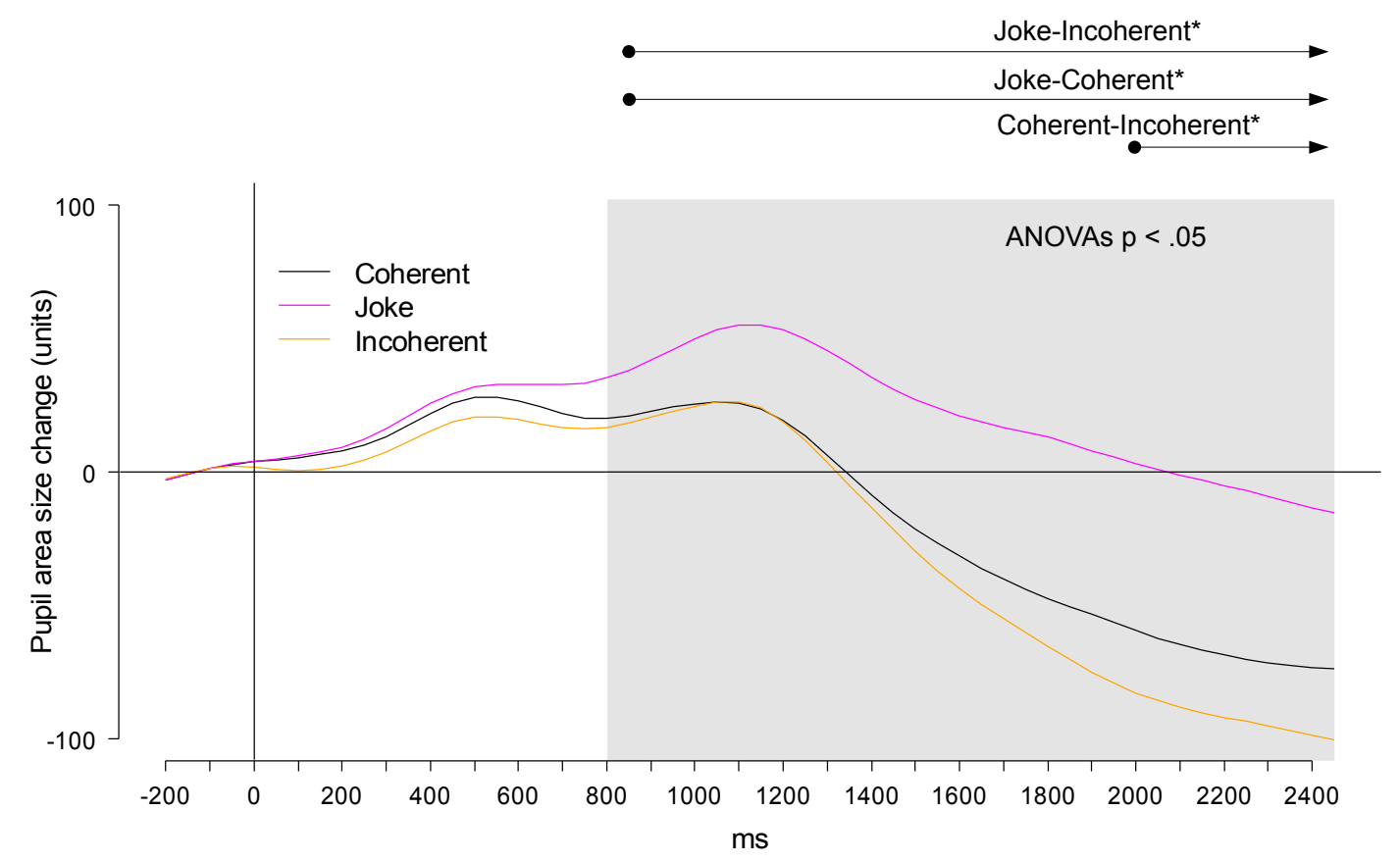

Figure 3.3: The grand average waves of the changes in pupil area (arbitrary unit) as a function of Time relative to the stimulus onset and Condition. The grey box indicates the time window with significant main effects of the running ANOVA for every $50 \mathrm{~ms}$. The arrows indicate the time windows with Bonferroni-corrected significant Post-hoc comparisons.

after the onset of the final word (PL). Compared to Incoherent, this N400 effect was reduced in the Joke condition. The N400 component is a reliable measurement for the degree of expectation violation and, even more important, for the degree of semantic integration difficulties. Therefore, the N400 effect here paralleled the predictability ratings of Experiment 1. The finding of reduced N400 effects to joke as compared to incoherent endings suggested a weaker incoherence and, probably, the activation of a possible alternative hidden joke interpretation. This activation might initiate a spreading activation toward new relevant semantic content for rapidly integrating the joke endings into the context. In contrast, for completely incoherent endings, such an activation and integration of a possible alternative interpretation might not occur.

In the time window of the hypothesized LLAN, i.e. 500 to $700 \mathrm{~ms}$ after the onset of 
the PL, no evidence for a LLAN component for joke endings was found. The LLAN component had been hypothesized to reflect increased working memory load necessary for the re-establishment of a coherent discourse or successful "frame-shifting" (Coulson \& Kutas, 2001; Coulson \& Lovett, 2004). Instead, we mainly found a sustained N400 effect for incoherent and - even though somewhat weaker - joke endings during this time interval. A possible reason for this contradictory finding might be the mere presence of incoherent endings. Incoherent endings might have been such a strong contrast that the perception of the difference between joke and coherent endings was strongly diminished. In addition, one might argue that the repetition of the text-fragments triggered the search for hidden joke interpretations also for incoherent endings. Accordingly, the LLAN might have been drastically reduced compared to previous studies (Coulson \& Kutas, 2001; Coulson \& Lovett, 2004). We directly tested for this possibility in Experiment 3.

In the time window of Late Positivity components (700 to $1000 \mathrm{~ms}$ ), joke endings elicited a frontal positivity compared to both coherent and incoherent endings. Importantly, the anterior locus of this ERP effect clearly differed from the typical posterior scalp distribution of a P600. Our finding of such late positivity with an anterior rather than posterior maximum parallels a previous report by Coulson and Lovett (2004) for right-handed women during joke comprehension. The authors related this finding to less hemispheric lateralization in women than in men. Even though participants' handedness and sex were not equally balanced in the present study, the present sample consisted for a big part of right-handed women. Thus, it cannot be excluded that a more heterogeneous sample would have led to a more posterior positivity.

Alternatively, it seems plausible that this frontal late positivity reflected emotional processes during joke comprehension, corresponding to the findings by Du et al. (2013). Accordingly, the frontal late positivity found here might be related to an "elabora- 
tion" stage of the joke comprehension (Wyer \& Collins, 1992), including emotional sub-processes towards the humorous stimuli, presumably generated in the vmPFC, bilateral amygdalae, and bilateral parahippocampal gyri (see Chan, Chou, Chen, \& Liang, 2012). Such interpretation is supported by our data from pupillometric recordings in two aspects. First, pupil diameters after the PL word started to dissociate between conditions around $850 \mathrm{~ms}$, thus covering the latency of the frontal late positivity in the Joke condition. Second and most importantly, pupil data parallel ERP findings: In both parameters, joke endings differed from both coherent and incoherent endings with larger pupil dilations and larger ERP amplitudes. Interestingly, pupil sizes for incoherent endings did initially not significantly differ from those to coherent endings. Starting later in time (around $2000 \mathrm{~ms}$ ), however, pupil dilations were diminished after incoherent compared to coherent endings. This finding suggested either a lack of cognitive processing effort or of emotional responses or even both in the Incoherent condition.

Together, two unexpected findings in our ERP data needed further clarification - the lack of LLAN effects to joke endings and of late positivities expected to occur after incoherent endings. Both insignificances might be due to contextual effects caused by the experimental setting, as we have discussed above. In order to control for such potential context effects on the ERP effects obtained here, we conducted two additional experiments in which either the joke or the incoherent condition were contrasted to coherent processing separately. Therefore, we omitted the Incoherent condition in Experiment 3 and the Joke condition in Experiment 4. 


\subsection{Experiment 3: ERP Comparison between Joke and Coherent}

The results regarding joke endings from Experiment 2 needed further exploration for several reasons. First, the previously reported enhanced LLAN to joke endings compared to coherent endings could not be replicated. Second, it could not be excluded that the mere presence of the incoherent endings affected the way participants processed the joke endings in a severe manner. In order to address these two points, Experiment 3 was carried out as a closer replication of a study by Coulson and Kutas (2001). Here, participants only received joke and coherent endings of the same text fragments. In addition, only right-handed participants were tested, and the distribution of male and female participants was equally balanced. We expected the following ERP effects: In comparison to coherent endings, joke endings should elicit enhanced amplitudes of the N400 and late positivities. If a mere presence of incoherent endings was responsible for the lack of a previously reported LLAN component, the LLAN should be elicited by joke endings under the current experimental conditions where incoherent endings were eliminated.

\subsubsection{Method}

\section{Participants}

Twenty-four students (12 females, 12 males) from different disciplines between 19 and 28 years $(M=23.42, S D=2.36)$ participated in this experiment. All of them were native speakers of German. They were all right-handed (Oldfield, 1971). All reported normal or corrected-to-normal vision and no neurological or neuropsychological disorders. Participants gave their informed consent and received $€ 15$ or course credit. None 
of them had participated in one of the previously described experiments.

\section{Material and Procedure}

Exactly the same stimulus material was used as in Experiment 2, but the Incoherent stimuli and the Incoherent filler items were excluded, resulting in three blocks with 64 trials each. Stimulus presentation followed procedures of Experiment 2.

\section{EEG recording and analysis}

Recording and pre-processing of the ERP data, including elimination of trials, artifact rejection and definition of ERP components (time windows and ROI electrodes), followed the same procedure as that in Experiment 2. For all participants, between $59 \%$ and $89 \%$ of the trials $(M=70.7, S D=14.18)$ remained for the analysis. ANOVAs on mean ERP amplitudes included the factors Electrode (9, 8, or 11, respectively) and Condition (2 levels - Joke, Coherent).

\subsubsection{Results}

\section{Behavioral data}

Test scores and demographic data are presented in Table 3.3. A paired samples t-test on Comprehension scores showed a significant effect of Condition, $t(23)=-6.11, p<$ $.001, d=1.13$, indicating worse comprehension of jokes in comparison to coherent stimuli. 
Table 3.3: Descriptive data of the behavioral variables in Experiment $3(N=24)$

\begin{tabular}{lcc}
\hline \hline Variable & $M$ & $S D$ \\
\hline Comprehension Total & 88.35 & 4.55 \\
Comprehension Coherent & 89.76 & 5.79 \\
Comprehension Joke & 80.64 & 8.37 \\
Number of Familiar Jokes & 6.71 & 5.93 \\
\hline \hline
\end{tabular}

\section{ERP data}

N400 (250-500 ms) The ANOVA of ERPs of the ROI, defined at central electrodes, revealed a significant main effect of Condition, $F(1,23)=8.60, p=.007, \epsilon=1, \eta_{p}^{2}=$ .272 , and no significant interaction between Electrode and Condition, $F(8,184)=1$.

LLAN (500-700 ms) The ANOVA for the ROI at left-anterior electrodes showed no significant main effect of Condition, $F(1,23)=1.71, p=.204, \epsilon=1, \eta_{p}^{2}=.069$, and no significant interaction between Electrode and Condition, $F(7,161)<1$. Since we found a sustained N400 effect for the Joke condition in Experiment 2, we tested for such effect by conducting an ANOVA of ERPs including central electrodes (as in Experiment 2). This analysis did neither reveal a significant main effect of Condition, $F(1,23)<1$, nor a significant Condition by Electrode interaction, $F(8,184)=1$.

Visual inspection of the data, however, indicated a peak of negative amplitudes for joke endings that was restricted to a few left-anterior electrodes and limited to the time window between 580 and $620 \mathrm{~ms}$ after the stimulus onset (see Figure 3.5). An ANOVA of mean ERPs at these selected anterior electrodes (F7, F5, FT7, and AF7) revealed an effect of Condition, $F(1,23)=4.89, p=.037, \epsilon=1, \eta_{p}^{2}=.176$, but no significant Condition by Electrode interaction, $F(3,69)<1$. This LLAN amplitude (aggregated over Condition and Electrode) was additionally significantly negatively correlated with participants' Total Comprehension score, $r(23)=-.58, p=.003$ (see Figure 3.4). 


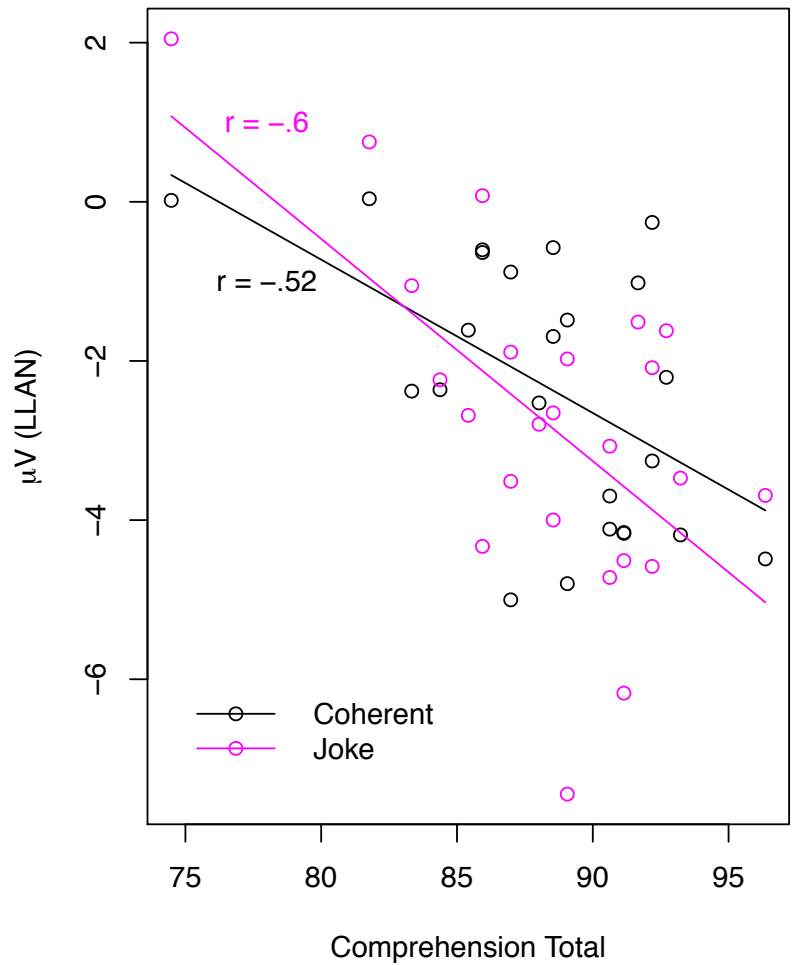

Figure 3.4: Scatterplot of the mean amplitude in the LLAN time window and its ROI and the total comprehension accuracy per participant split up for each condition in Experiment 3. Regression lines indicate the significantly negative linear relationship between these two variables.

Late Positivities (700-1000 $\mathbf{~ m s ) ~ T h e ~ A N O V A ~ f o r ~ a n t e r i o r ~ R O I ~ e l e c t r o d e s ~ r e v e a l e d ~}$ neither a significant main effect of Condition, $F(1,23)=1.99, p=.172, \epsilon=1, \eta_{p}^{2}=.080$, nor a significant interaction between Electrode and Condition, $F(8,184)<1$.

\subsubsection{Discussion}

First of all, it is striking that the effects obtained in Experiment 3 were - in general - much weaker than the effects in Experiment 2, even though the same stimuli and the same procedure had been used. There were two differences between these two ex- 


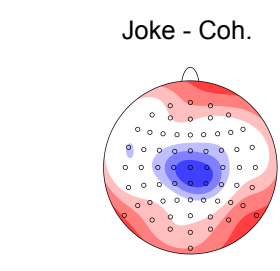

$250 \mathrm{~ms}-500 \mathrm{~ms}$

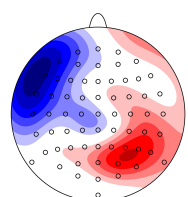

$580 \mathrm{~ms}-620 \mathrm{~ms}$

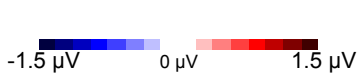

AF7

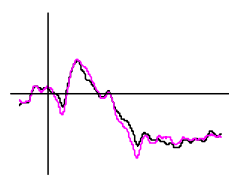

C5

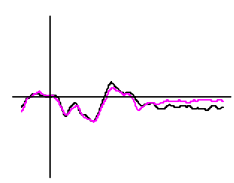

P5

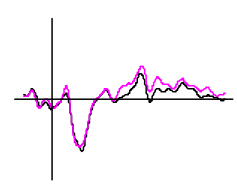



Cz

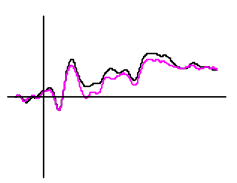

Pz

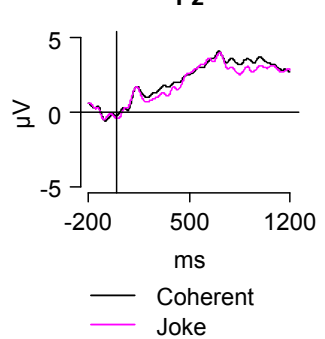

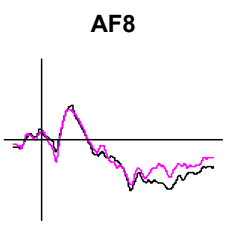

C6

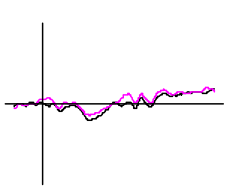

P6

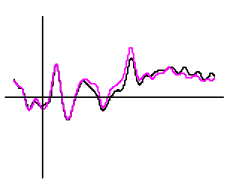

Figure 3.5: Difference maps of the conditions for the three time windows and grand average waves for selected electrodes as a function of Time relative to the stimulus onset and Condition in Experiment 3.

periments: (i) the absence of the Incoherent condition and the Incoherent fillers and (ii) a slightly different sample of participants (right-handed and equally balanced between males and females). However, neither visual inspection nor statistical tests of the data revealed sex-related differences. The first modification reduced the duration of the experimental session and might have also made the design of the experiment more obvious.

The only robust effect in Experiment 3 was the N400 effect at centro-parietal electrode sites. Overall, it was weaker than in Experiment 3. Since there were no incoherent endings, participants might have found it easier to semantically integrate the joke ending which led to less comprehension difficulties. Visual inspection of the data revealed similar activation patterns in the following time windows compared to Experiment 2 
and to the literature (left anterior negativity and late frontal positivity). Statistical analysis, however, showed no convincing evidence for a negativity for the Joke condition at left-anterior electrode sites between 500 and $700 \mathrm{~ms}$ (LLAN effect), nor for the frontal positivity between 700 and $1000 \mathrm{~ms}$. We did find a left anterior negativity between 580 and $620 \mathrm{~ms}$ after stimulus onset that was similar to the a priori expected component. Additionally, amplitudes of this component were related to participants' total comprehension ability. Nevertheless, the reliability of this finding is highly questionable due to possible inflations of the type 1 error by the post-hoc selection of the time window and the electrodes.

\subsection{Experiment 4: ERP Comparison between Inco- herent and Coherent}

In Experiment 2, incoherent endings as compared to coherent endings elicited larger and long-lasting N400 effects as hypothesized but failed to evoke any late positivity. The data, however, showed a tendency for a left anterior late positivity which was almost significant. The comparison between the Incoherent and Coherent condition might have been affected by the presence of the joke endings. The N400 effect was as predicted, but the left anterior positivity between 700 and $1000 \mathrm{~ms}$ after the stimulus onset was puzzling. This positivity was clearly different from a typical P600 effect. Two explanations for this frontal positivity in Experiment 2 seemed reasonable. On the one hand, it could be the case that the context of the humorous stimuli in Experiment 2 has severely altered the processing of incoherent endings. It might have caused participants to engage in the vain effort of searching a coherent interpretation for a nonsensical discourse. Such unsuccessful attempts are plausible if participants could not be completely sure that the incoherent endings were really incoherent or just joke endings that were very 
hard to grasp. On the other hand, this component might have reflected the confusion after an incoherent discourse. Experiment 3 aimed to shed light on this question by removing all the joke endings, and also the incoherent filler endings.

\subsubsection{Method}

\section{Participants}

Twenty-four students (12 females, 12 males) of different disciplines between 19 and 29 years $(M=22.79, S D=2.43)$ participated in this experiment. All of them were native speakers of German and right-handed (according to Oldfield, 1971). All reported normal or corrected-to-normal vision and no neurological or neuropsychological disorders. Participants gave their informed consent and received $€ 15$ or course credit. None of them had participated in one of the previously described experiments.

\section{Material}

Exactly the same stimulus material was used as in Experiment 2, but the Joke stimuli and the Incoherent filler items were excluded (three blocks with 64 trials per block).

\section{Procedure}

was exactly the same as in Experiment 2.

\section{EEG recording and analysis}

Recording and pre-processing of the ERP data followed Experiment 2. None of the data had to be removed from subsequent analyses. Between $58 \%$ and $100 \%$ of the trials 
Table 3.4: Descriptive data of the behavioral variables in Experiment $4(N=24)$

\begin{tabular}{lcc}
\hline \hline Variable & $M$ & $S D$ \\
\hline Comprehension Total & 87.92 & 4.74 \\
Comprehension Coherent & 89.65 & 4.91 \\
Comprehension Incoherent & 78.15 & 8.91 \\
\hline \hline
\end{tabular}

$(M=94.36, S D=8.7)$ remained after artifact rejection. ERP components of interest (N400, sustained N400, and late positivities), respective time windows (250-500 ms, 500-700 ms, and 700-1000 ms) as well as selected ROI electrodes for N400 effects were identical to Experiment 2. Since we expected typical P600 effects for incoherent endings under the given experimental conditions, we additionally assessed ERP activity from a group of posterior electrodes (Iz, Oz, P1, Pz, P2, P9, P7, P5, P3, PO3, PO7, O1, P10, P8, P6, P4, PO4, PO8, O2). ANOVAs on mean ERP amplitudes included the factors Electrodes (9, 9, or 19, respectively) and Condition (2 levels - Incoherent, Coherent).

\subsubsection{Results}

\section{Behavioral data}

The test scores are presented in Table 3.4. A paired samples t-test with Condition as a within factor and Comprehension score as the dependent variable showed a significant effect of Condition, $, t(23)=-7.5, p<.001, d=-1.13$. The comprehension score of Incoherent was significantly lower than the score of Coherent.

\section{ERP data}

N400 (250-500 ms) The ROI ANOVA revealed a significant main effect of Condition, $F(1,23)=56.94, p<.001, \epsilon=1, \eta_{p}^{2}=.712$, and a weaker but significant interaction between Electrode and Condition, $F(8,184)=2.46, p=.049, \epsilon=.51, \eta_{p}^{2}=097$. As 
can be seen in Figure 3.6, incoherent endings elicited enhanced N400 amplitudes over central electrodes, similar to Experiment 2.

Sustained N400 (500-700 $\mathbf{~ m s ) ~ T h e ~ R O I ~ A N O V A ~ s h o w e d ~ a ~ s i g n i f i c a n t ~ m a i n ~ e f f e c t ~}$ of Condition, $F(1,23)=19.54, p<.001, \epsilon=1, \eta_{p}^{2}=.459$, but no significant interaction between Electrode and Condition, $F(8,184)<1$. Similar to Experiment 2, an enhanced N400 occurred within this time window (see Figure 3.6).

Late positivities (700-1000 ms) The ROI ANOVA for the posterior electrode cluster revealed a significant main effect of Condition, $F(1,23)=9.27, p=.006, \epsilon=1, \eta_{p}^{2}=$ .287, but no significant interaction between Electrode and Condition, $F(18,414)=$ $1.19, p=.304, \epsilon=.426, \eta_{p}^{2}=.050$.

\subsubsection{Discussion}

In line with our hypotheses, the main findings of this control experiment consist of a long-lasting N400 and an enhanced late positivity to incoherent as compared to coherent endings. Whereas the N400 effects show high similarity to those obtained in Experiment 2, indicating their independence from experimental context, the late positivity clearly differs between both experiments. Whereas in Experiment 2, incoherent endings failed to elicit a significant late positivity, as became evident from both the overall and the ROI ANOVA, enhanced positivities occurred over posterior electrode sites in response to incoherent endings. Possibly, this change is related to the fact that no joke endings were present in the current setting. Therefore, participants realized very early that it does not make sense to attempt to find a new interpretation. 


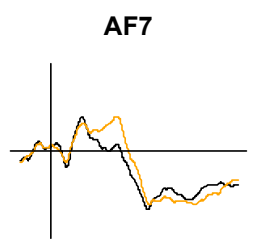

C5

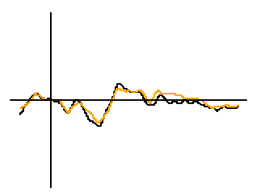

P5
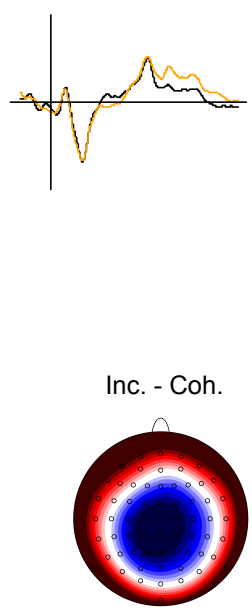

$250 \mathrm{~ms}-500 \mathrm{~ms}$
AFz

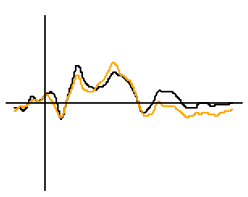

$\mathrm{Cz}$

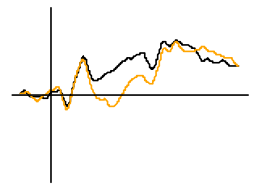

$\mathrm{Pz}$

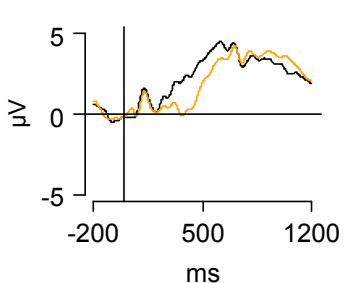

- Incoherent

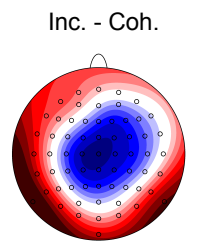

$500 \mathrm{~ms}-700 \mathrm{~ms}$

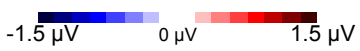

AF8



c6

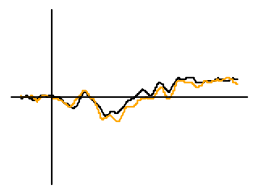

P6



Inc. - Coh.

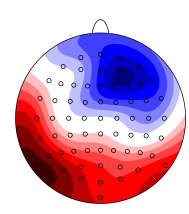

$700 \mathrm{~ms}-1000 \mathrm{~ms}$

Figure 3.6: Difference maps of the conditions for the three time windows and grand average waves for selected electrodes as a function of Time relative to the stimulus onset and Condition in Experiment 4.

\subsection{General Discussion}

GP jokes were described as a semantic-pragmatic phenomenon. They exploit a misleading discourse comprehension mechanism in order to amuse the recipient. The mental representation of the discourse based on the initially dominant interpretation is violated at the punch-line. Through a spreading activation of relevant semantic networks an 
alternative interpretation of the discourse is re-established. This re-interpretation and semantic re-analysis is called belief revision in the present account in order to highlight its close relationship to probabilistic (conditional) human reasoning and non-monotonic logic (Mayerhofer \& Schacht, 2013). A successful belief revision in combination with the humorous content of the hidden interpretation is emotionally rewarded with mirth. Four empirical studies (reading time, three ERP studies, and pupil size data) supported the hypothesized neuro-cognitive processes when compared to coherent texts on the one hand and to (discourse-)incoherent texts on the other hand: violation of the semantic representation, revision of the semantic representation and emotional reaction.

The GP joke endings differed compared to the same texts with a coherent ending. They were harder to grasp, were rated as less predictable, triggered increased reading times for the final word due to the detection of the semantic violation and due to the belief revision of the semantic mental representation. Further, the joke endings elicited the well-established N400 effect in the ERP data reflecting some (minor) difficulties at the stage of the semantic integration. This effect provides evidence for an automatic default interpretation of the ambiguous set-up. Contrary to the findings by Dwivedi, Phillips, Einagel, and Baum (2010) who found no evidence for the commitment to one interpretation with semantically ambiguous texts, the present findings indicated such a commitment: Participants committed to one dominant interpretation of the ambiguous textual input, rather than remaining undecided about the underspecified or misleading discourse. This initial incoherence led to the experience of incongruity which has been pointed out as a key element in the perception of humorous stimuli (e.g., Forabosco, 1992; Giora, 1991; McGhee, 1979; Nerhardt, 1977; Suls, 1972).

In the processing stages following the N400 effect, we found mixed evidence that was strongly affected by the context. In Experiment 2, joke endings also elicited a weak sustained N400 effect. In Experiment 3, however, this sustained N400 did not occur with 
the absence of incoherent endings in the experimental setting. In both experiments, we found no convincing evidence for the LLAN effect for the joke endings. Nevertheless, in Experiment 3, when incoherent endings were absent from the experimental design, there was a peak of negative amplitudes at left anterior electrodes around $600 \mathrm{~ms}$ after the stimulus onset. The amplitude of this negativity additionally was significantly affected by participants' general comprehension ability. Coulson and Kutas (2001), and Coulson and Lovett (2004) had previously found a stronger LLAN effect for better comprehenders and an enhanced N400 effect for poor comprehenders. Together with the present findings, it is not reliably possible to argue for the existence of this component. If and that remains the question - there is a LLAN component that truly reflects additional processing effort during discourse comprehension, or in particular the semantic re-interpretation necessary for the re-establishment of a coherent discourse during joke comprehension, then this component is characterized by three points: It is (i) weak in effect size, hence not reliably elicited in experiments; (ii) strongly susceptible to contextual influences that disable participants to engage in additional processing effort for successful comprehension; for example in Experiment 2 the presence of incoherent endings might have sufficed to make participants think that a joke ending offers no reinterpretation, which resulted in a sustained N400 effect instead of LLAN activity; and (iii) strongly susceptible to individual discourse comprehension ability; activity of this component might not be restricted to joke comprehension ability.

Contrary to previous findings with joke material (Coulson \& Lovett, 2004; Marinkovic et al., 2011), no evidence was found for the hypothesized (posterior) P600 component. We could not find any hint for a similarity between the well-studied (mainly syntactic) GP sentences and the (mainly semantic) GP jokes. Nevertheless, we did find a frontal late positivity for the joke endings in Experiment 2 which was diminished and nonsignificant in Experiment 3. It appeared to be comparable to the frontal P600 which 
had been found for ambiguous and complex syntactic sentence parsing (Kaan \& Swaab, 2003). It also was similar to a component reported for jokes that involved frame-shifting. In these findings, the component was mainly present for right-handed women (Coulson \& Lovett, 2004). Nevertheless, the interpretation of this component remains unclear and might also be related to the emotional reaction (see below).

Participants in the Joke condition were emotionally rewarded with the experience of mirth. This was indicated by the rating of the humorous potential just as well as by the larger pupillary response starting from $850 \mathrm{~ms}$ after the onset of the final word. Pupil dilation is known to accompany participants' cognitive load and emotional arousal. Since the pupil dilation in the current experiment was significantly higher only for the joke endings, but not for the incoherent endings, we argue that this result mainly was based on the emotional reaction. Incoherent endings were assumed to need cognitive processing effort for the detection of the incoherence, just as for a possible revision attempt. If the larger pupil dilation for the joke endings was related to the cognitive load plus the emotional reaction this would mean that the absence of larger pupil dilation for the incoherent endings was due to a lack of higher cognitive processing effort. This interpretation, however, was contradicted by the longer reading times for the incoherent endings compared to the coherent endings. Therefore, larger pupil dilation for the joke endings was mainly based on the emotional reaction of mirth. The emotional reaction might have also been reflected by the differences in the Late Positivity mean amplitudes at prefrontal electrode sites in Experiment 2. This frontal positivity became predominant for the joke endings in the same time window as the significantly larger pupil dilations. Previously, emotional reactions to joke stimuli or to other emotional verbal stimuli had been related to late positivity components which were similar to the present results (Du et al., 2013). Nevertheless, typical late positivities related to an emotional reaction usually have a more posterior distribution. Therefore, the present interpretation of the 
frontal positivity remains speculative. No ratings of the subjective experience of mirth for the individual participants were carried out in this study. Comparing such ratings to the amplitude of the frontal positivity component could help to find better evidence for such an interpretation. The precise functional equivalent and the reliability of the frontal positivity still remain unclear given the current evidence.

The comparison of the joke endings with the incoherent endings was new in the present study. Incoherent endings were harder (or impossible) to be semantically integrated in the discourse. This was also reflected by longer reading times compared to the coherent endings, better comprehensibility in the ratings study, and mainly by a stronger and more sustained N400 component compared to both the joke and the coherent endings. Further, the incoherent endings showed a context sensitive activation pattern of the late positivity components, a pattern which also remains unclear given the current evidence. Also, the incoherent endings did show the smallest pupil dilation, probably related to the lack of an emotional response, and probably also indicating lower cognitive load compared to the coherent endings and to the joke endings (see above). This suggests that the processing of totally incoherent endings differs from the processing of the joke endings. The differences in the N400 component indicate that the processing starts to diverge at a very early stage of the comprehension, namely right at the stage of the word recognition. While the joke endings very early seemed to trigger the search for the alternative interpretation and the revision of the semantic representation, as reflected by the reduced N400 effect, this process is either absent or less emphasized for the incoherent endings. Following processing stages of incoherent endings strongly depended on the context. The mere presence of the joke endings in Experiment 2 seemed to have altered the processing after the detection of the semantic incoherence compared to the processing of the very same endings in Experiment 4. In the latter experiment, incoherent endings elicited a typical posterior P600 activation. One possible explanation 
could be that the absence of joke endings made it clear to the participants that no coherent explanation was provided. Accordingly, participants were better able to engage in monitoring strategies that signal the impossibility of a successful comprehension, possibly reflected by the posterior P600 activity. A similar functional equivalent of the P600 component had previously been proposed by Van Herten et al. (2005).

Limitations of the study have to be pointed out. The whole lab situation is an artificial setting. Participants had to sit in a computer lab or, as for the ERP experiments, in a dark EEG lab. A large amount of short texts had to be read on a computer screen in a way which might be very distinct from natural reading processes (moving windows paradigm, RSVP), and especially from the usual social interaction of joke telling. Furthermore, participants read every text fragment three times or two times respectively in the control studies. Even though we balanced out the order of the conditions for the participants, this repetition probably had an impact on the processing of all three conditions. However, this design had the advantage of keeping all experimental settings apart from the crucial final word between the three conditions completely identical. Moreover, all experimental results were aggregated across 48 different stimuli, assuming a fairly homogeneous stimulus material. Despite the focus on GP jokes, which is a more precise definition and scope of the stimulus material than in previous studies related to verbal humor, the ratings suggested quite a high variance within the stimuli in terms of predictability, comprehensibility, and humorous potential. An in depth-analysis on the stimulus level might enrich the present results with a more fine-grained understanding of the processes in relationship to inherent stimulus features.

Further research should address more precisely the functional relationships of the described ERP components. Especially the interpretation of the frontal positivity, which was found in Experiment 2 for joke endings compared to both to coherent and to incoherent endings remains speculative. Since joke endings in the present study were different 
to the other conditions by two features, the revision and the emotional response, the component could be related to any of these two processes. Siebörger (2006) carried out an fMRI study in order to disentangle the cognitive processes of joke comprehension from the emotional reactions. He compared GP jokes (revision plus emotional reaction) to texts which he called "Revisionsgeschichten (revision texts)" (revision but no emotional reaction), to straight coherent (no revision needed) and to incoherent texts (no coherence, no revision, no emotional reaction). The results indicated differences for all three hypothesized processes. A similar design as an ERP study would be a very useful next step. Future research could also investigate the nature of the N400 during joke comprehension in more detail by manipulating the salience of the initially dominant interpretation (possible increase of the N400 effect) and the accessibility of the hidden interpretation (possible increase of the LLAN component or reduction of the N400 effect) of the joke endings by contextual priming prior to the stimulus presentation (Mayerhofer \& Schacht, 2013).

The present experiments hardly allow distinguishing the theoretical account of frameshifting (Coulson \& Kutas, 1998; Coulson, 2001) from the focus on the revision, as in the present account. Both accounts share the general idea of a re-interpretation process. This re-interpretation is in line with the assumption of incremental and nonmonotonic reasoning processes based on inferential belief updates and permanent and active constructions of situation models during language comprehension in order to coherently represent the meaning of a text (Baggio et al., 2008). Even if an interpretation once has been established, it does not mean that this interpretation cannot be overridden anymore by an alternative explanation which is better able to integrate the new textual evidence. 


\section{Chapter 4}

\section{Investigating Salience and Accessibility}

\subsection{Introduction}

In the present study, we were mainly interested in how contextual constraints can affect neuro-cognitive processing of a GP joke by referring to two crucial features of these texts: salience and accessibility (see below). Take (1) as an example for a GP joke.

(1) I would love to die like my grandfather, peacefully and calm during sleep (SU). Not frightened, screaming, and suffering like his passengers (PL). (Attributed to Bob Monkhouse ${ }^{1}$ )

In (1), the first sentence provides the SU. The final word "passengers" of the second sentence is the crucial disambiguating element of the PL and will thus be referred to as the PL. Default interpretation of the SU leads to the mental representation that the grandfather died in his bed since this is the most plausible scenario given the cues from the text. Under the assumption of this initially dominant interpretation (SU1) the information from the PL triggers the detection of an incoherent discourse since the causal connection between the two sentences remains unclear. Accordingly, the initial interpretation is detected to be wrong and has to be substituted by an alternative

\footnotetext{
${ }^{1}$ Retrieved from http://www.bitcomedy.co.uk/comedy-features/101-short-jokes/ onthe4thof June, 2013.
} 
hidden joke interpretation (SU2), "the grandfather died while driving a public bus". This change of the semantic representation in addition to the sudden shift from one frame of knowledge to a different one, called "frame-shifting" (Coulson, 2001) or "script-switching" (Attardo \& Raskin, 1991), appears to be a crucial element for the humorous potential of this huge subclass of verbally expressed humor; especially in combination with some absurdity or "inappropriateness" (Ritchie, 2004, p.61) of the hidden interpretation.

Evidence for these assumed sequential (sub-)processes of discourse comprehension comes from various studies which investigated the processing of jokes with stimulus material that is highly comparable to what is called GP jokes in the present account. Vaid et al. (2003) demonstrated priming effects on the reaction times in lexical decisions that were specifically related to the different stages of joke comprehension. Lexical decisions of words that were semantically related to the non-joke interpretation were faster before the occurrence of the set-up, while lexical decisions of joke-script related words were faster right after the occurrence of the punch-line. This was taken as evidence that a specific non-joke script (Attardo \& Raskin, 1991), e.g., the bed room setting applied to example (1), was predominantly activated during the SU, while the joke-script, e.g., traffic setting, was activated right after the occurrence of the PL. Further, self-paced reading times (Coulson \& Kutas, 1998), eye-fixation data (Coulson et al., 2006), and ERP data (Coulson \& Kutas, 2001; Coulson \& Lovett, 2004; Coulson \& Williams, 2005) strongly suggest semantic integration difficulties and higher processing effort for the comprehension of jokes compared to semantically coherent non-joke endings, assumed to reflect processes of frame shifting as described above.

Two important features of GP jokes are the "degree of salience" (Giora, 2003) of the initially predominant interpretation and the "degree of accessibility" of the hidden in-

\footnotetext{
${ }^{2}$ Ariel $(1988,2001)$ introduced the term accessibility as an important concept for the comprehension of referring expressions in discourses. The term, with the meaning it is intended here, has been applied to the comprehension of jokes before (De Palma \& Weiner, 1992; Wyer \& Collins, 1992) and also to
} 
terpretation.

In a recent theoretical account on salience and accessibility of GP jokes, Mayerhofer and Schacht (2013) defined salience as a probabilistic function of linguistic and non-linguistic cues of the initial part of the text (SU). The meaning construction in addition to the explicit linguistic input also involves implicit and automatic inferences, mainly concerning space, time, causation, protagonists, and objects (Zwaan \& Radvansky, 1998). Giora (2003) described the salience of a specific meaning in the context of a clearly identifiable expression (e.g. an ambiguous word) as a function of conventionality, familiarity, frequency, and prototypicality (Rosch, 1999). Whenever there is ambiguity, these factors decide which of the possible meanings is fore-grounded, i.e. highly salient, in a recipient's mind. In a similar manner, Goatly (2012, p. 276ff) pointed out the influence of collocation patterns and concordance data on the salience of one interpretation. What we call here the GP mechanism, Goatly referred to as "false priming" (Goatly, 2012, p.276ff). Following that approach, a specific ending of a text should become highly predictable mainly because of the surrounding words. Frequency measures of co-occurrences of surrounding words with the ambiguous element accordingly should account for the degree of salience. In GP jokes, however, the salience of an interpretation very often is based on a complex chunk of words and sentences (see Dynel, 2009, p.222ff). It is not always a clearly identifiable expression. Hence, salience of the initial interpretation of a GP joke often cannot be easily accounted for by corpora-based statistics. It is also empirically supported that contextual effects on word recognition due to "predictive inferences" in discourse comprehension are more strongly affected by the message-level of the discourse rather than by collocation patterns of semantically related words (R. K. Morris, 1994). The underlying inferential processes integrate a wide range of sources of knowledge (linguistic knowledge, pragmatic knowledge, world

the comprehension of metaphors (Werning, Fleischhauer, \& Beseoglu, 2006). 
knowledge, social knowledge, etc.). Recently, Mayerhofer and Schacht (2013) modeled salience in GP jokes as reasoning processes that rely on conditional probabilities of a Bayesian Network-based knowledge representation, independently from the type of knowledge to be activated. The default reasoning process leads to the initially dominant interpretation and its semantic representation as a situation model (Van Dijk \& Kintsch, 1983). This first interpretation, respectively its situation model, is the one with the least processing effort and the highest ranked plausibility given an individual's subjective knowledge, context, memories, and experiences. In this sense, the salience of an interpretation can vary between different individuals but can also vary within one individual depending on different contexts or time points. Accessibility (Ariel, 1988, 2001; De Palma \& Weiner, 1992; Wyer \& Collins, 1992) - when applied to the phenomenon of GP jokes - refers to the ease of retrieval of knowledge which can accommodate the semantic irregularity of the PL (see Mayerhofer \& Schacht, 2013). After a recipient encounters the semantic incoherence, a new set of explanations for the whole discourse needs to be found. In many cases, the activation of an alternative interpretation involves other characters' higher-order belief representations, shifting from one semantic field to a completely different and contrasting semantic field (frame shifting), (causal) bridging inferences, or engagement of specific (insider) world knowledge. For obvious reasons, accessibility, despite being presented as a feature of the textual input, also can be modulated by recipients' inter-individual differences, such as specific world knowledge, the ability of mind-set switching, or the amount of exposure to verbal humor. All these factors together determine the ease of finding the hidden joke interpretation for the recipient. Similar to the degree of salience, another important factor on the degree of accessibility is the context of the joke. Applied to example (1), presenting the joke in a setting that strongly activates knowledge related to bedroom or a hospital, the salience of the initial interpretation should become increased. In contrast, presenting this joke in 
a setting which strongly pre-activates knowledge on traffic or accidents should increase the degree of accessibility of the hidden interpretation.

We hypothesized that salience and accessibility are two distinguishable factors, which jointly affect the characteristics of processing GP jokes. However, even though both factors were assumed to interplay in GP joke comprehension, we claimed that the direct influences from these two factors could be experimentally tested. Since salience is a feature of the initially dominant interpretation, we assumed that the degree of salience affects the degree of the initial incoherence after encountering the PL. The higher the degree of salience, the stronger is the perception of the violation of the semantic coherence at PL. Accessibility, on the other hand, is a feature of the alternative interpretation and we assumed that it mainly affects the ease of retrieval of the relevant knowledge necessary for the hidden interpretation. Consequently, accessibility is associated with the ease of revision of the situation model in order to re-establish a coherent meaning of the otherwise incoherent discourse. The higher the degree of accessibility, the easier it is to carry out the necessary belief revision by finding a new set of explanations which can accommodate the irregularity of the PL.

\subsection{Experiment 5: Contextual Constraint on Inter- pretation Processes}

In the present study, we manipulated salience and accessibility by pre-activating either the initially dominant or the alternative hidden interpretation. If participants are prior to the GP joke - presented with a word which is semantically related to the initial interpretation, semantic content linked to the initial interpretation (SU1) should be activated. Therefore, this prime condition should directly increase salience and also 
indirectly decrease accessibility. Priming the alternative interpretation (SU2), on the contrary, should directly increase accessibility and also indirectly decrease salience. A neutral, i.e. semantically unrelated word should not directly affect salience or accessibility. However, neutral words might be misinterpreted as cues for alternative interpretations under the given boundary conditions of the present experimental manipulations. In this case, the neutral word might indirectly decrease accessibility and, in addition, also salience (see Figure 4.1(c) for a schematic representation of the design and experimental manipulations).

We applied a self-paced moving window paradigm (Just et al., 1982). By doing so, the reading time for every single word could be measured. Reading times are an accumulation of several processing stages, ranging from recognition of a given word to higher comprehension processes, such as the integration of a word with its co-text and context. For GP jokes, the reading times of the PL (and possibly of a follow-up sentence) were supposed to index the processing effort and the additional working memory load during the discourse comprehension process necessary for the successful joke comprehension as outlined in the introduction. Final words of a sentence or a clause usually are associated with longer reading times due to "wrap-up effects" (Just \& Carpenter, 1980; Rayner, Kambe, \& Duffy, 2000; Rayner, Sereno, Morris, Schmauder, \& Clifton Jr, 1989), traditionally described as the consequence of sentence internal integration processes. Some preceding reading units cannot be completely processed until the final word appears. Thus, the full processing is then completed with the final word. In addition, previous research showed that comprehension difficulties can have a delayed effect. Rather than increasing the reading time of the final word, there can be "spill-over" effects on reading units that appear after the crucial target word. These belated spill-over effects are an indicator of more complex comprehension issues due to persistent inconsistencies at previous reading units (Calvo \& Castillo, 1996; Just et al., 1982; Pexman, Ferretti, \& 
Katz, 2000).

Since the final word of the last sentence of GP jokes is the disambiguating element (PL), at this reading position, the semantic incoherence occurs and belief revision processes are initiated. Higher salience was hypothesized to lead to a stronger violation of the semantic coherence, whereas higher accessibility should lead to an easier revision. Both processes should be additional factors of the "wrap-up" and accordingly were assumed to take place mainly immediately following the lexical recognition of the PL. Therefore, we mainly expected an influence of the manipulation at the reading position of the PL. An increase of the salience should lead to longer PL reading times while an increase of the accessibility should lead to shorter reading times at the PL both compared to the neutral condition. Spill-over effects on the reading times of a follow-up sentence should reflect later processing stages of the revision of initially dominant semantic representations. Since the revision was hypothesized to be related to the degree of accessibility, we predicted that higher accessibility should lead to shorter reading times at the position of the follow-up sentence.

Given the hypothesized sequential nature of GP joke comprehension, we expected no influence of the manipulation on the reading times of the set-up part of the text. Prior the PL, the hidden joke interpretation should not be maintained. Thus, any manipulation regarding the hidden interpretation should be ineffective at this stage of the joke. Together, we were interested in the reading times of three reading positions: the SU, the final word of the PL, and the average reading time of the follow-up sentence (FS). 


\subsubsection{Method}

\section{Participants}

Forty-six participants were tested. One participant had to be excluded due to a comprehension accuracy of less than $35 \%$ and due to highly deviant reading time patterns, together indicating that the participant just clicked through the experiment without proper processing of the texts. Remaining participants (32 females, 13 males) ranged in age between 19 and 40 years $(M=24.36, S D=4.15)$. All were native German speakers and students of the University of Göttingen. They were reimbursed with course credits or $€ 5$. They had not participated in any experiment with the same stimulus material.

\section{Material}

Forty-five GP jokes in German were used as the stimulus material. In all jokes, the final word was the crucial disambiguating element of the PL. Each joke was additionally followed by a neutral sentence continuing the general topic of the joke. Single prime words were selected according to a text-linguistic analysis of the GP stimuli. A SU1 prime was a word which was semantically related to the first dominant interpretation of the set-up of the joke. A SU2 prime was a word which was semantically related to the second hidden interpretation of the set-up. Neutral words were chosen without any strong relationship to possible interpretations of the set-up. This is an example stimulus including the primes and the comprehension question (stimulus taken and adapted from Dynel, 2009, p.276):

- Prime: SU1: Diät (Diet) SU2: Kerker (Jail) Neutral: Auto (Car)

- GP joke: Ich habe noch immer den Körper einer 18-Jährigen. Und zwar in meinem Keller. (I still have the body of an eighteen-year-old. It is in my cellar.) 
- FS: Außerdem gibt es im Keller auch Ratten. (There are rats in the cellar as well.)

- Comprehension questions: Die Person ist ein Mörder. (The person is a murderer.) (Yes/No).// Die Person hat einen gutgebauten Körper. (The person is in good shape.) (Yes/No).

Three different sets of stimuli were created, counterbalancing the prime-GP combinations. There was no GP joke repeated for a participant.

\section{Procedure}

The experiment was carried out in a group lab on a computer with groups of two to five participants per session. After they had indicated the demographic data, participants were instructed on the computer screen that they participated in an experiment on text comprehension. They were made familiar with the presentation of the stimuli and were told to carefully read the texts. Prior to the experimental trials, participants had to complete three practice trials with GP jokes not overlapping with the experimental stimuli. Each trial started with a fixation cross presented for $100 \mathrm{~ms}$ after the participant had pressed the space bar. Next, the prime word appeared for $1500 \mathrm{~ms}$ on the center of the computer screen, followed by a second fixation cross displayed for 500ms. Then, the GP joke and the follow-up sentence were presented with an adapted version of the self-paced moving window paradigm (Just et al., 1982), implemented by Pygame, a graphical interface for Python. First, the whole GP joke was presented, with the whole final sentence of the joke and the follow-up sentence being masked by blanks. This sentence appeared word-by-word with separate button presses for each word. Only the actual word appeared unmasked, whereas the preceding word became masked again. After completion of the joke sentence the follow-up sentence was presented in the same way (see Figure 4.1(b) for a screenshot of the presentation of the final sentence of the 
joke and the follow-up sentence). Each trial was followed by a comprehension question which was randomly assigned to be answered with yes or no using the arrow keys.

Each participant completed a set of 45 trials consisting of 15 trials per condition (SU1 prime, SU2 prime, Neutral), in fully randomized order. Figure 4.1(a) gives an example of a complete trial.

\section{Analysis}

A priori we were interested in three reading time variables, defined as our regions of interest (T1, T2, and T3). T1 was the reading time of the whole text fragment before the final sentence (SU). T2 was the reading time of the final word (PL), measured as the time between the onset of the word and the button press to proceed with the follow-up sentence. T3 was the average reading time of the follow-up sentence (FS), measured as the average of the times between the onset of the words of the follow-up sentence and the respective button presses. All reading time data ( 45 x $45=2025$ observations) were checked for plausibility. Visual and descriptive analyses revealed influential outliers in both directions. Thus, all observations $<200 \mathrm{~ms}$ and $2.5 S D$ above each individual participant's mean were excluded for the dependent variables, T1 (exclusion rate: 7.36\%), T2 $(3.46 \%)$, and T3 $(2.96 \%)$. The trimmed data were aggregated by participant and condition. These aggregated mean reading times in ms (per participant and condition) were subjected to separate within One-Way-ANOVAs on T1, T2, and T3, respectively. Effect sizes are reported as generalized eta square (Bakeman, 2005). We carried out Bonferroni-corrected two-tailed t-tests for dependent groups for pair-wise comparisons. Cohen's $d$ is reported as effect size on the average $S D$ from two means corrected for dependence between means, using Morris and DeShon's (2002) equation 8. 
(a)

$$
\text { Fixation [100ms] }
$$

Prime $[1500 \mathrm{~ms}]$

Fixation [500ms]

Garden path joke

+ Follow-up sentence

Comprehension Question

(b)

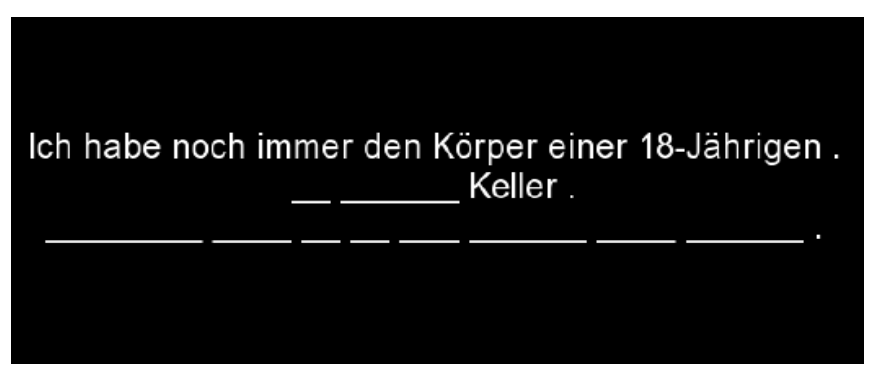

(c)

\begin{tabular}{|c|c|c|}
\hline SU1 prime: & salience & accessibility \\
\hline SU2 prime: & salience & accessibility \\
\hline Neutral prim & alience & accessibility \\
\hline
\end{tabular}

Figure 4.1: (a) A schematic representation of the time course of the sequential presentation of a complete trial. (b) A screenshot of the self paced moving window presentation of the example stimulus in German at the reading position of PL. (c) A schematic representation of the design and the manipulations. Bold indicates a direct influence; non-bold indicates only an indirect influence. The arrows indicate an increase or a decrease on the salience/accessibility. 


\subsubsection{Results}

\section{Comprehension Score}

Overall, $74.85 \%$ of the comprehension questions were answered correctly. Comprehension accuracy did not differ across conditions $(M \mathrm{~s}=74$ to $76 \%, S D \mathrm{~s}=43$ to $44 \%)$. Neither a One-way within ANOVA nor a non-parametric Friedman-test indicated that there was any effect of type of priming on comprehension accuracy.

\section{Reading times}

Descriptive data are presented in Table 4.1 and Figure 4.2. ANOVAs revealed a significant effect of Priming condition on T2, $F(2,88)=3.63, p=.031, \eta_{G}^{2}=.076$, and T3, $F(2,88)=3.99, p=.021, \eta_{G}^{2}=.083$, but not on T1, $F<1$. According to post-hoc t-tests, reading times at the PL (T2) were significantly shorter after SU2 priming compared to SU1 priming, $t(44)=-2.88, p=.006, d=-.440$. Reading times of the FS (T3) were significantly shorter after SU2 priming compared to Neutral, $t(44)=-2.89, p=.006, d=-.479$. All other comparisons did not reach statistical significance, although there was a trend for shorter reading times at the $\mathrm{PL}$ after SU2 priming compared to Neutral, $t(44)=-1.77, p=.083, d=-.239$, and for shorter reading times of FS after SU2 priming compared to SU1 priming, $t(44)=-1.55, p=.129, d=-.242$.

\subsubsection{Discussion}

The present study aimed at investigating contextual influences on several stages of discourse comprehension in GP jokes. To this aim, we implemented a priming paradigm in which single words either increased the salience by priming the initially predominant 
Table 4.1: Descriptive data of the reading times in Experiment $5(N=46)$

\begin{tabular}{|c|c|c|c|}
\hline Priming & Condition & $\overline{\bar{M}}$ & $\overline{C S D}$ \\
\hline \multicolumn{4}{|c|}{ (a) Set-up } \\
\hline SU2 & & 5633 & 2081 \\
\hline Neutral & & 5623 & 1864 \\
\hline SU1 & & 5636 & 2329 \\
\hline \multicolumn{4}{|c|}{ (b) Punch-line } \\
\hline SU2 & & 1505 & 975 \\
\hline Neutral & & 1581 & 1053 \\
\hline SU1 & & 1628 & 1017 \\
\hline \multicolumn{4}{|c|}{ (c) FS } \\
\hline SU2 & & 553 & 138 \\
\hline Neutral & & 586 & 178 \\
\hline SU1 & & 573 & 171 \\
\hline
\end{tabular}

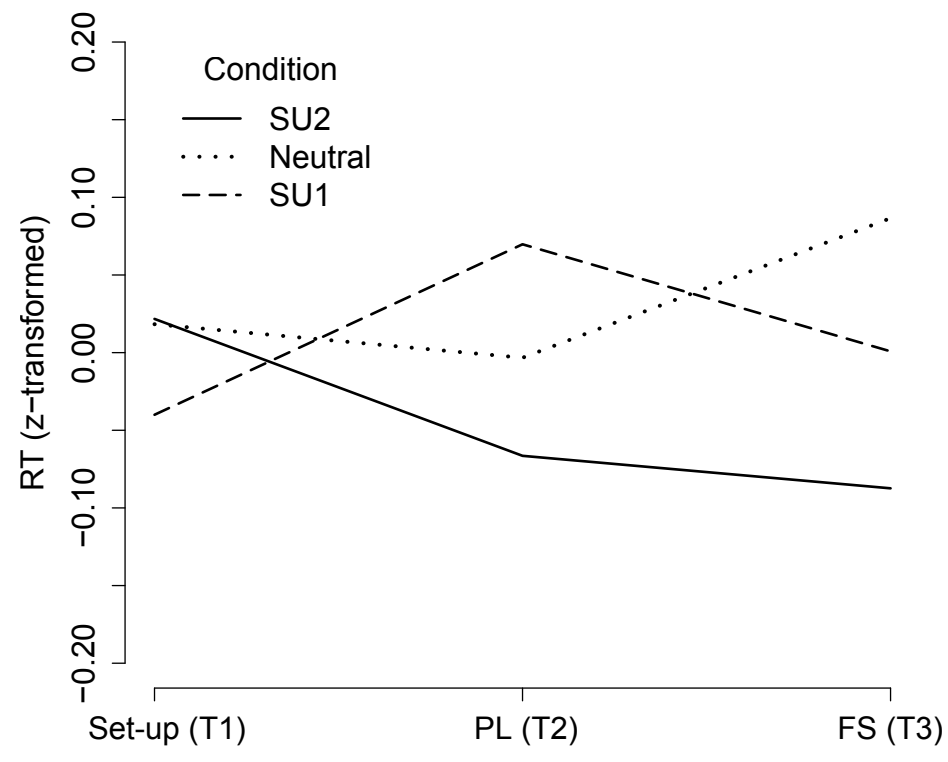

Figure 4.2: Z-transformed mean reading times (trimmed and aggregated across participants and condition) of words as a function of Priming Condition and the three regions of interest: Set-up (reading time of the whole text fragment $=\mathrm{T} 1$ ), PL (final word $=$ $\mathrm{T} 2$ ), and FS (average reading time of the words of the follow-up sentence $=\mathrm{T} 3$ ).

interpretation or increased the accessibility by priming the hidden alternative interpretation. Neutral words were unrelated to both conditions. Analyses of reading time data 
at different regions of interest (T1-set up; T2 -critical PL word; T3 -average reading time of the follow-up sentence) revealed the following finding: First, no influence of the priming manipulation was found on the reading times of the text fragment, the set-up of the joke (T1), indicating the same processing of the SU independently from the priming condition. Second, reading times at the PL (T2) were significantly longer after priming of the initial interpretation (SU1) as compared with priming of the hidden joke interpretation (SU2). Third, in contrast, the neutral condition led to significantly longer reading times of the follow-up sentence when compared to the priming of the joke interpretation.

These results mainly supported our hypotheses regarding the two relevant features of GP joke processing - salience and accessibility. As outlined in the introduction, salience refers to the degree of dominance of an initial default interpretation of a potentially ambiguous discourse. Accessibility refers to the ease of detecting the hidden interpretation and the retrieval of knowledge which allows integrating the new linguistic evidence in order to re-establish a coherent semantic representation. Priming SU1 was hypothesized to directly increase salience and to indirectly decrease accessibility. Priming SU2 should directly facilitate the degree of accessibility and indirectly decrease salience. Accordingly, we hypothesized shorter reading times of PL words for SU2 priming compared to both neutral condition and SU1 priming. As expected, SU2 primed endings were read significantly faster compared to SU1 primed endings. However, we found only a strong tendency, but no significant difference between the reading times at the PL of SU2 primed and neutral endings.

As outlined in the introduction, reading time data for the final word presumably reflect an accumulation of several processing stages involved that can be pre-lexical (e.g. visual perception of the letters, the detection of the orthographical pattern), lexical (e.g., selection of lexical candidates), or post-lexical (e.g., integration of the word into its 
preceding discourse and context) (e.g., R. K. Morris, 1994). There is evidence that contextual constraints affect predictive inferences and anticipation-based word recognition on a pre-lexical or lexical level (Calvo \& Castillo, 1996; Calvo, 2000; R. K. Morris, 2006; Van Berkum, Brown, Zwitserlood, Kooijman, \& Hagoort, 2005). Thus, one plausible impact of SU2 priming could be a faster lexical access of the PL word in this condition. Such a mechanism should be restricted to the condition of increased accessibility, i.e., SU2 priming, since there was no direct relationship between (non-)prime and PL words in the other conditions (SU1 prime and neutral). Importantly, such lexical priming would have a functional locus prior to the processing stages suggested to be specifically relevant for GP joke comprehension, i.e., detection of incoherence and revision processes, but it might subsequently facilitate these more complex comprehension processes at later stages. Accordingly, recognition facilitation at a pre-/lexical level might partly account for significantly shorter PL reading times for SU2 priming. However, such mechanism cannot explain why the effects of SU2 priming compared to the neutral condition occurred later in time, i.e. as spill-over effects at the follow-up sentence. This specificity in the time course of the effects needs to be addressed by considering potential post-lexical influences.

Spill-over effects are usually associated more strongly with post-lexical processing stages or with a lack of comprehension given its persistence and delayed and long-lasting occurrence (see Just et al., 1982, p.232ff). For example, Pexman et al. (2000) reported evidence for enhanced spill-over effects for the additional comprehension effort of sarcastic interpretations of metaphors, also using a moving window paradigm. These authors argued that the spill-over effects are related to the "settling of inconsistencies and comprehension issues that have not been completed earlier in the processing sequence" (Pexman et al., 2000, p.219). We suggest similar higher-level comprehension mechanisms to be responsible for the spill-over effects in the present experiment. Our data 
suggest that our experimental manipulations affected the time course of the comprehension and the initiation of the revision process.

Therefore, the remaining important question concerns the differences between the SU1 vs. SU2 comparison and the neutral vs. SU2 comparison. Calvo (2000) reported evidence for a relationship between the degree of contextual constraint and the dynamics of facilitation effects of predictive inferences over time in a word naming paradigm. Higher semantic constraint was accompanied by earlier facilitation effects of predictive inferences. A similar process might be responsible for an earlier effect of the contextual constraint in the SU1 primed condition compared to the delayed effect in the neutral condition. The SU1 prime enhanced the semantic constraint by increasing the salience of the initial interpretation, leading to stronger predictive inferences. Accordingly, the violation of the coherence directly at the PL position was emphasized. This emphasis might have prompted participants to re-analyze the semantic representation of the discourse leading to longer wrap-up effects right at the final word (PL). In the neutral condition, the semantic constraint remained constant compared to the SU2 primed condition, while accessibility was lower compared to the SU2 condition, delaying revision processes. Thus, the difference between the neutral condition (low salience and low accessibility) and the SU2-primed condition (low salience and high accessibility) did not become statistically significant until only after the PL, that is at the follow-up sentence. Further research is needed to clarify the observed differences between the manipulations of the degree of salience on the one side and the degree of accessibility on the other side. If the present interpretation is correct and the present reading time patterns reflect an altered time course of the joke comprehension due to different semantic constraints, one could expect a comparable pattern of effects on ERP components which had previously been related to the processing of jokes, namely the N400 component (word recognition, detection of incoherence, and semantic integration difficulties) and a late left-anterior 
negativity component (revision, frame-shifting, enhanced working-memory related processes) (see Coulson \& Kutas, 2001; Coulson \& Lovett, 2004).

One major limitation of the present study is the artificial setting and the unnatural reading format, not to mention the lack of a social and oral context in which a joke usually is embedded. For instance, the self-paced-moving window technique used in our experiment does not allow for saccades, regressions or skippings, which typically occur with skilled readers (see e.g., Just et al., 1982; Just \& Carpenter, 1980). Therefore, a follow-up study with the tracking of eye-gaze durations could help to get a clearer picture of the involved processes. Also, the repetition of 45 GP jokes might have familiarized the participants with the underlying mechanisms of interest, possibly obscuring natural joke comprehension processes. Moreover, the comprehension questions were aimed at asking for the semantic representation of the discourse without baring their function to the participants. As a consequence, these questions were not related to specific single elements of the previous discourse but rather gave ample space for individual interpretations. This characteristic might explain the overall low comprehension accuracy found in the present study. Self-reports from participants indicated that they occasionally did not answer the comprehension questions in the "correct" way despite having correctly understood the joke.

Despite these weaknesses, the present results indicate the importance of contextual constraint during discourse comprehension on two distinguishable, yet dynamically interacting, features of discourses that exploit the GP mechanism. The manipulation of the contextual constraint led to either a manipulation of the degree of salience of the initial interpretation or to a manipulation of the accessibility of the hidden interpretation. Most importantly, distinguishable manipulations resulted in an alteration of the time course of the hypothesized sequential processing stages. This indicates that joke comprehension, and discourse comprehension in general, can be significantly altered 
depending on the type of manipulation that is carried out by contextual constraints. Previous research on priming effects mainly dealt with the direction from mental representations of a discourse (i.e. on the message level) to word recognition (see R. K. Morris, 2006, for an overview). The current study investigated the reversed direction and highlighted the effect of contextual priming on the construction and revision of these mental representations. 


\section{CHAPter 5}

\section{General Conclusion}

\subsection{Empirical Evidence: Quasi-Replication and New Findings}

One aim of the dissertation project was finding empirical evidence for the hypothesized sequential nature of GP joke processing. This processing focused on three core stages: detection of the violation of coherence, revision of the semantic representation, and emotional reaction. The present data backed up previous findings related to joke and humor processing reported by Coulson and Kutas (1998, 2001); Coulson (2001); Coulson and Lovett (2004). (i) Reading time data revealed higher working memory load for joke endings compared to coherent endings. Joke endings need more processing effort than its coherent versions. This significant difference in the reading time of the final word was assumed to be a cumulative measure of the first two processing stages (i.e. the detection of the violation, and the revision; perhaps also including the emotional reaction). (ii) An enhanced N400 component presumably indicated the detection of the incoherence, respectively the semantic integration difficulty, following the lexical recognition of the punch line word. Subsequent ERP components could not be backed up to the same extent. An enhanced left anterior negativity had been previously reported and had been speculated to be related to the re-interpretation stage and to the ability to comprehend a joke by carrying out the frame shifting (Coulson, 2001; Coulson \& Lovett, 2004). Accordingly, the component was hypothesized to reflect higher working memory 
engagement. Empirical findings of this component could only be partially replicated. The present data were not convincing, and such a component was not clearly evoked by joke endings compared to coherent endings. Nevertheless ERP data in Experiment 3 showed a similar activation pattern compared to previous studies for a very restricted time window and scalp distribution. Also, the relationship between this component and comprehension ability was backed up by the present results. Activation patterns of a possible posterior P600 were not found at all in the present data for the processing of GP jokes. However, some mixed evidence for a possible frontal P600 was presented. This activation pattern needs much further exploration in terms of its reliability and its functional aspect.

New empirical data in this dissertation project mainly involved three findings: (i) the comparison of joke endings to totally incoherent endings, (ii) the pupil dilation data, (iii) and the manipulation effects of contextual constraint on the reading time of GP joke endings and its follow-up sentence. As for the first aspect, reading time data and the ERP data clearly indicated initially similar activation patterns between joke endings and incoherent endings, although ERP data indicated that semantic integration difficulty was significantly lower for joke endings. This implies that at a very early stage, starting after $250 \mathrm{~ms}$ after the reading of the punch-line, joke endings presumably led to spreading activation of relevant knowledge that eased the integration of the ending compared to a totally incoherent ending. These activation patterns, however, were even more strongly diverging at later processing stages. While incoherent endings also took longer to be processed by participants compared to coherent endings, a stronger and sustained N400 component for incoherent endings compared to joke endings accompanied the lack of a successful reinterpretation and accordingly a lack of comprehension. Additionally contextual influences (the experimental setting) strongly affected later processing stages of the incoherent endings. The mere presence or absence of joke endings altered the 
neuro-cognitive activity in the time window after a sustained N400 component (from $700 \mathrm{~ms}$ after the stimulus onset onwards). This alteration was presumably related to the experimental setting. In Experiment 2 participants could not know for sure whether a given ending of a text was simply incoherent and impossible to be semantically integrated or whether it was a joke ending for which no alternative interpretation had been found yet. Consequently, participants might have started the same revision processes as for GP joke endings, but failed at finding an alternative interpretation. Reading time data of Experiment 1 indicated quite a persistent willingness to search for an alternative interpretation even in the absence of such a reinterpretation possibility. In Experiment 3, incoherent endings were probably recognized as what they were, and the ERP activity showed indications of a typical posterior P600 pattern. This finding speaks for the role of the P600 as a monitoring component which signals the reader about the correctness of their reading process (Van Herten et al., 2005).

Pupil size data of Experiment 2 revealed that joke endings led to significantly larger pupil dilations than both coherent and incoherent endings starting from around 800 ms after the appearance of the punch-line. This difference was characterized by huge effect sizes and by a long-lasting nature (until $3000 \mathrm{~ms}$ after the onset and possibly even longer). Especially, the correlation between the average pupil dilation (aggregated by participants) of a stimulus with the average rating of the humorous potential of a stimulus (see Appendix C) strongly backs up the interpretation that pupil dilation is associated with the emotional reaction as a consequence of a humorous stimulus (see below for implications of this effect).

The manipulation of contextual constraint in Experiment 5 and its alteration of the time course of the sequential processing stages of GP jokes can be considered as empirical evidence for core theoretical assumptions of Chapter 2. It was hypothesized that salience and accessibility are two important concepts on the side of stimulus features that influ- 
ence the processing of GP jokes. Both factors were hypothesized to be susceptible to the contextual setting of a GP joke. The experimental manipulation either increased the availability of semantic content related to the initially dominant interpretation or the availability of semantic content related to the hidden alternative interpretation. It was hypothesized that either the detection of the coherence violation or the re-interpretation and belief revision process would be affected. Even though the present results indicated that both factors are highly interacting, the usefulness of this conceptualization was established. Mainly, a general processing advantage was found for an increase of the accessibility of the hidden meaning. This advantage of an increased accessibility manifested itself earlier compared to trials with increased salience as compared to trials with salience being kept constant.

\subsection{Implications}

Theoretical elaboration of the hypothesized processing of verbal GP humor was the other aim of this dissertation project. This aim was mainly addressed by the second chapter. The processing of GP jokes was embedded in a probabilistic frame work of discourse comprehension. It was modeled as inferential reasoning with the goal of meaning construction. The recipient tries to maintain a coherent representation of an ambiguous textual input. These probabilistic reasoning processes were hypothesized to be carried out in an intuitive and automatic manner. Only the statistically most plausible interpretation given contextual, linguistic, and knowledge related cues is represented. This automatic interpretation, however, can be retracted as a consequence of new surprising and incongruent textual input. In an incremental and non-monotonic manner, new knowledge can be retrieved. Based on this newly retrieved knowledge, the initial interpretation is substituted by an alternative hidden interpretation that can better explain 
the new input. These sequential and basic processes had been suggested in different terms before in humor theories (e.g., Attardo \& Raskin, 1991; Ritchie, 2004; Suls, 1972) just as in theories of discourse comprehension (e.g., Baggio et al., 2008). However, the focus on a specific subclass of verbal humor allowed the processes to be narrowed down and modeled in more detail. The crucial aspect of the present theoretical account was the focus on the revision of the semantic representation, referred to as "belief revision". This focus distinguished it from other linguistically oriented accounts on joke processing and also from the cognitive-linguistics approach of frame-shifting (Coulson, 2001), even though the latter concept can be considered as highly similar, and also was integrated in the current model. The frame-shifting account seems to imply that actual shifting from one frame to another is always necessarily involved. In the present account, frame shifting was not considered to be the core element of the processing but only a possible additional factor of the belief revision process. This focus on the belief revision is in line with the neuro-cognitive theory put forward by Hurley et al. (2011) who have claimed that humor has developed as an emotional reward for overcoming committed false beliefs. The present empirical data, however, do not allow to distinguish the present account from previous accounts, since most incongruity based accounts on humor share these basic sequential processing assumptions and can hardly be differentiated by their empirical predictions.

One new theoretical development of the dissertation projection was the conceptualization of probabilistic salience and accessibility. They were at the core of the presented model. Predictions derived from these considerations were tested empirically and partly confirmed in Experiment 5. GP Jokes are not a totally homogenous class of stimuli. Subtle differences in the linguistic features of these stimuli can strongly influence the way they are processed. Salience and accessibility might be important factors that have to be acknowledged for the investigation of humor. Different manipulations of these 
two factors led to different reading time patterns. Nevertheless, the strong relationships between various factors that have been modeled in a fairly independent manner indicate the importance of acknowledging a highly interactive network of stimulus features and the neuro-cognitive processing of these features. Disentangling these highly interacting factors can only be achieved in a limited way for the purpose of empirical investigations. Separating and isolating them can be fruitful for a more detailed understanding, but any artificial operationalization aiming for such a dissociation will only partially reflect the true nature of the comprehension process of GP jokes and verbal humor in general.

The focus of this dissertation project was the cognitive mechanisms involved in the comprehension process. Many questions regarding the humorous potential of GP jokes had been left out from the empirical investigations, as well as from the theoretical elaborations. The main reason for this was the sheer complexity of the phenomenon of humor. A stimulus that is reliably perceived as humorous usually combines many different characteristics striving for a synergetic effect. The display of superiority (Gruner, 2000), tension relief due to taboos, sexual content, aggression (Freud, 1905), a dissociated and playful state of mind - as opposed to a goal-oriented state of mind - (Apter, 1989) and many other factors have been suggested in the literature as essential characteristics of a humorous experience. On top of it, humor is usually embedded in a social and nonthreatening context (Gervais \& Wilson, 2005). Therefore, many more factors related to this social interaction and to the performance style will come into play. Within the current frame work, all these factors could best be considered as "additional flavors" of a specific stimulus. They have to be isolated in the same manner as the GP mechanism in the current approach in order to allow valid conclusions about their workings. Certainly, it is very rewarding to focus on these factors. This dissertation project aimed to omit them in order to get a clearer picture and empirical support for the mechanisms that are related to the GP phenomenon. 
Furthermore, the elaboration and development of a methodological tool box for the investigation of GP jokes was a subgoal of this aim. Empirical correlates that have been shown to be useful psycholinguistic research methods for this purpose were: rating studies, reading time data, ERP data, pupil dilation, and manipulations of the contextual constraint.

The rating data presented in Experiment 1 and the intercorrelations between the ratings (see Appendix C) indicated a strong relationship between the predictability of the ending and the comprehensibility of the joke on the one hand, and between both of these factors with the humorous potential of a joke on the other hand. Figure 5.1 depicts a graphical overview of the theoretical concepts and the empirical correlates that might be together responsible for the processing of GP jokes. All of these factors strongly interact. Accordingly, strong relationships between these factors and these correlates are expected and need to be acknowledged. Still, methodological improvements of the items used for a rating study could help to achieve a stronger dissociation of salience and accessibility, and possibly of other concepts that might be helpful for the dissection of this dynamic interaction.

The self-paced reading time data of Experiment 5 highlighted the importance of separating the reading time of the final word (pre-lexical, lexical and wrap-up stage) of the text and the reading time of a follow-up sentence. The latter measure variable was considered to be more strongly related to higher-level discourse processing. Furthermore, the priming of a specific interpretation by contextual cues emerged to be a fruitful experimental manipulation. Previously, priming studies had only investigated the priming effect of a specific interpretation during joke comprehension on the lexical recognition of a word (Vaid et al., 2003).

In terms of ERP components, the most suitable and robust measure variable for the investigation of GP jokes emerged to be the N400 component, thus backing up previous 
investigations of highly similar stimulus material (Derks et al., 1997; Coulson \& Kutas, 2001; Coulson \& Lovett, 2004; Coulson \& Williams, 2005). A component that can be described as a late left anterior negativity (LLAN) was further investigated under the assumption that it is a functional equivalent of the additional processing effort necessary for the successful comprehension of GP jokes. This investigation did not lead to convincing evidence. In addition to previous findings (Coulson \& Kutas, 2001; Coulson \& Lovett, 2004), however, the present data pointed towards a more general role of a possible LLAN component. The component might be associated with discourse comprehension, not only relevant for joke and humor comprehension. Given this interpretation it might be fruitful to relate the component to other neuro-cognitive correlates that have been suggested for discourse comprehension processes. fMRI studies have revealed that search for coherence and coherence building during discourse comprehension is accompanied by increased activation in the dorso-medial prefrontal cortex (dmPFC) (Siebörger, Ferstl, \& von Cramon, 2007). Recently, Mossbridge, Grabowecky, Paller, and Suzuki (2013) have been able to predict comprehension accuracy in reading comprehension by ERP data that were transformed via a current-source-density index. Visual inspection of their presented untransformed data in the appendix also revealed a left anterior negativity related to the comprehension effort.

Lastly, the present data showed the high relevance of the investigation of pupil dilation during the comprehension of verbal humor. Pupil dilation was strongly associated with the humorous potential of GP jokes, presumably with the emotional reaction to humorous stimuli in general. GP jokes in Experiment 2 led to huge effects of higher pupil dilation. The strong correlation between the ratings of the humorous potential and participants' average pupil size in response to a given stimulus (see Appendix C) additionally backed up this interpretation. Together, these findings underline the importance of pupil dilation as a measure variable for future investigations of humorous 
stimuli for a wide range of research questions related to the emotional reaction to humor. This emotional reaction has been referred to as mirth (Martin, 2007), amusement, or exhilaration (Ruch, 1993). Earlier research had shown that exposure to humor can affect psychophysiological parameters like skin conductance and heart rate (Averill, 1969; Godkewitsch, 1976), loss of muscle tone (Overeem, Lammers, \& Van Dijk, 1999), and other parameters (see for a review on cardiovascular, respiratory, and electrodermal measures Kreibig, 2010, p.406). To my knowledge, pupil dilation had not been shown as a result of exposure to humorous stimuli. Importantly, the psychophysiological changes related to humor are not mere side effects of laughter. They are assumed to stem from increased sympathetic nervous system activation due to the emotional elaboration of the stimuli (Martin, 2007, p.163). Increased pupil dilation has also been assumed to stem from increased sympathetic nervous system activation (Bradley et al., 2008).

Gervais and Wilson (2005) have suggested that humor and laughter have evolutionarily developed within the context of social play. Laughter served as a signal to the group that a non-threatening, safe environment allows the engagement in playful behavior and partial detachment from goal-oriented behavior (Apter, 1989). In line with the "broaden-and-build-theory" of positive emotions (Fredrickson, 1998; Fredrickson \& Branigan, 2005), they further argue that this emotion-induced behavior led to adaptive advantages in terms of the strengthening of cognitive, physical, and social resources. However, the basic functions of laughter and humor were co-opted in many ways. As argued by Hurley et al. (2011), this emotional reaction in terms of the cognitive resources seems to be especially associated with the detection and revision of committed false beliefs, since quick adaptation to changing and surprising new environments is one of the crucial aspects of survival and fitness. A GP joke can be considered as playful, non-serious and non-goal-oriented communication that allows to practice these evolutionarily developed benefits within the realm of language and text comprehension, thus 


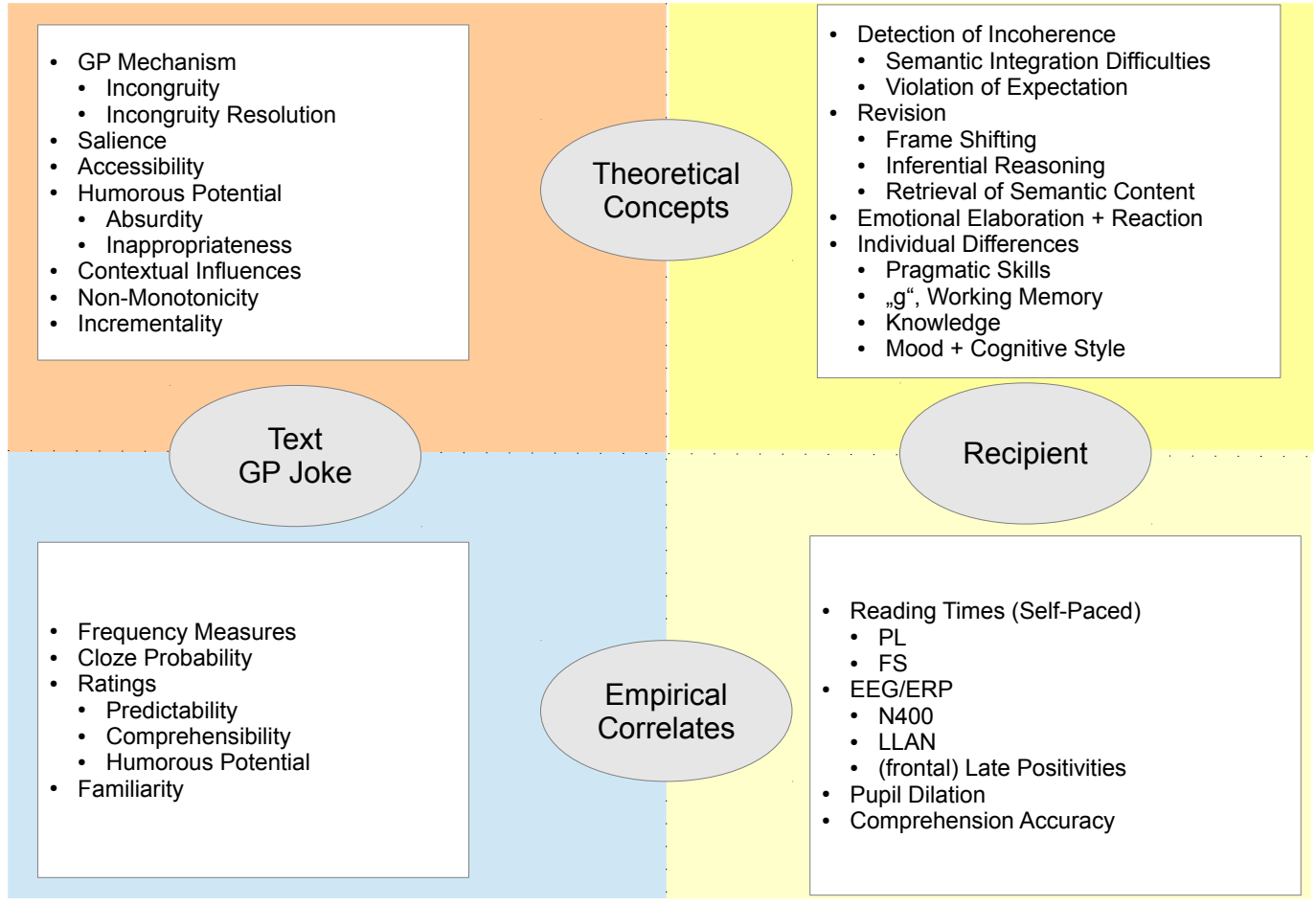

Figure 5.1: Text and GP joke comprehension as interaction between a stimulus and a recipient. Theoretical concepts on both sides of this interaction and its empirical correlates investigated, supported, and suggested within the dissertation project.

triggering the emotional reaction of mirth.

\subsection{Future Direction}

Future studies on verbal humor in general and on GP jokes could investigate in more detail the influence of participant features. In Chapter 2, it has been assumed that working memory skills (mind set switching, retrieval of knowledge, monitoring skills) are highly involved in the processing of GP jokes. Also, pragmatic skills, cognitive style, or verbal intelligence have been hypothesized to strongly affect the way a humorous stimulus is perceived and elaborated. Operationalizations of these concepts can shed more light 
on the hypothesized processing and seem feasible to be carried out. The current data, together with previous findings, pointed towards a relationship between comprehension accuracy and a specific ERP component that manifested itself as a (late) left anterior negativity. Comprehension accuracy, however, is only a very indirect auxiliary variable. Presumably it reflects a combination of the above mentioned theoretical concepts of participant features. Isolating these concepts from each other will be a useful next step for empirical investigations of joke processing.

Rating studies and reading time data in future investigations could be adapted in order to reflect more precisely the theoretical concepts that were depicted in Chapter 2. Particularly, hypotheses derived from the conceptualization of salience and accessibility could be addressed by items that allow to better distinguish between cognitive processes that are a consequence of features affecting the salience of the initially dominant interpretation and those that affect the accessibility of the hidden joke interpretation. The present results, however, indicate that salience and accessibility strongly interact with each other, so maybe such a distinction might not be achieved at all.

In general, ERP investigations will have to explore in more detail the functional equivalents of the found ERP activation patterns. The N400 component was hypothesized to be related to stimulus features (predictability of the punch-line, salience of the dominant interpretation, contextual constraint) and to participant features (exposure to verbal humor, contextual priming, pragmatic skills) that together determine how strongly a recipient is committed to one specific dominant interpretation. In a similar manner, the late left anterior negativity was hypothesized to be related to factors that affect the ease of retrieval of semantic knowledge necessary to successfully re-establish a coherent interpretation of the initially incoherent discourse. But more empirical data are needed to get a clear picture regarding this hypothesized relationship. Evidence in this dissertation project concerning that matter was mixed. Last but not least, late positivities 
- especially the frontal P600 - need further investigation. Currently, interpretations regarding functional equivalents of this component remain highly speculative. One possible interpretation was that this component could be related to the stage of emotional elaboration (Wyer \& Collins, 1992; Du et al., 2013). If this assumption is correct, participant ratings directly after each trial concerning the emotional reaction of mirth towards a presented joke should be associated with such an ERP activation pattern. Data in this dissertation project did not point towards such a relationship.

In regards to the emotional processing of GP jokes and humorous stimuli, future investigations can apply pupil dilation data as a reliable measure variable of the emotional reaction, amusement or mirth. The humorous potential of a GP joke was described as a combination of incongruity that is based on the GP mechanism and of secondary incongruity that is transported via the content of the plot or story of a GP joke. Many research questions that had been omitted in the current dissertation project due to its focus on the cognitive mechanism could be addressed in more detail by experimental designs that make use of pupil dilation as the dependent variable. Factors that had been neglected as irrelevant "additional flavors" of the jokes in respect to their semantic content, such as the display of superiority, sexual content, taboos, tension relief, etc. could be manipulated. Such an operationalization could allow the investigation of the influence these factors have on the humorous potential of the stimulus. Previous investigations on verbal humor, just as the present dissertation project, have helped to understand in more detail the cognitive processing of verbal humor and the emotional reaction triggered merely by the cognitive mechanism. The most relevant question in the field of humor research, however, that still - some people might add fortunately - remains highly mysterious and magical given the current state of research, could be addressed with this variable: What is it that makes a joke really funny? 


\subsection{Concluding Remarks}

The processing of GP jokes was investigated as a dynamic interaction between a reader and a text as a stimulus that carries the potential to be perceived as humorous by the reader. Features and concepts on both sides of this interaction have been elaborated on a theoretical level and empirically challenged in a series of experiments. Some of the hypothesized processes and relationships within this modeled interaction have been empirically well supported, some need further clarification, while others have been modeled in order to be addressed in more detail in future investigations. Text comprehension and humor comprehension are both research fields that carry great difficulties because of the complexity of factors that are contributing to their workings. By focusing on a specific mechanism, and by limiting the research to small units of research objects that are feasible and operationalizable for experimental settings, however, this dissertation project could help to get a better understanding of this complex interaction between a reader and a short humorous text like a GP joke. Despite the brevity of a GP joke, the present findings possibly can be extended to bigger discourses, texts, or films that exploit this mechanism on a larger scale. Findings of this project mainly underline one essential characteristic of the human mind related to humor. The cognitive system incrementally uses cues in the environment in order to build up an internal mental representation of the ongoing (textual) input. Unexpected surprising input that does not fit the internal mental representation triggers non-monotonic revision processes that can lead to a quick adaptation of this representation. Within a playful state of mind, these processes can be practiced by humor and joke comprehension. The benefits of this engagement usually are socially and intellectually rewarding. These benefits are the reasons why humor is so ubiquitous and usually highly appreciated in the behavior and experience of humans. 


\section{References}

Apter, M. J. (1989). Reversal theory: Motivation, emotion and personality. Taylor \& Frances/Routledge. 3, 108, 111

Ariel, M. (1988). Referring and accessibility. Journal of Linguistics, 24(1), 65-87. doi: $10.1017 / \mathrm{S} 002222670001156786,88$

Ariel, M. (2001). Accessibility theory: An overview. In J. S. Ted Sanders \& W. Spooren (Eds.), Text representation: Linguistic and psycholinguistic aspects (pp. 29-87). Amsterdam: John Benjamins. 86, 88

Ashby, F., Isen, A., et al. (1999). A neuropsychological theory of positive affect and its influence on cognition. Psychological Review, 106(3), 529-550. doi: 10.1037/ 0033-295X.106.3.529 4, 42

Ashby, F., Velentin, V., \& Turken, A. (2002). The effects of positive affect and arousal on working memory and executive attention. In S. Moore \& M. Oaksford (Eds.), Emotional cognition: From brain to behaviour (pp. 245-287). Amsterdam: John Benjamins. 4

Attardo, S., Hempelmann, C. F., \& Di Maio, S. (2002). Script oppositions and logical mechanisms: Modeling incongruities and their resolutions. Humor, 15(1), 3-46. doi: 10.1515/humr.2002.004,January2006 34

Attardo, S., \& Raskin, V. (1991). Script theory revis (it) ed: Joke similarity and joke representation model. Humor, 4, 293-347. doi: http://dx.doi.org/10.1515/ humr.1991.4.3-4.293 13, 86, 107, 134

Averill, J. R. (1969). Autonomic response patterns during sadness and mirth. Psychophysiology, 5(4), 399-414. doi: 10.1111/j.1469-8986.1969.tb02840.x 111 
Bach, K. (1984). Default reasoning: Jumping to conclusions and knowing when to think twice. Pacific Philosophical Quarterly, 65(1), 37-58. Retrieved from http:// online.sfsu.edu/kbach/defaultreasoning.pdf 22

Baggio, G., Van Lambalgen, M., \& Hagoort, P. (2008). Computing and recomputing discourse models: An ERP study. Journal of Memory and Language, 59(1), 36-53. doi: http://dx.doi.org/10.1016/j.jml.2008.02.005 48, 84, 107

Bakeman, R. (2005). Recommended effect size statistics for repeated measures designs. Behavior Research Methods, 37(3), 379-384. doi: 10.3758/BF03192707 94

Bayer, M., Sommer, W., \& Schacht, A. (2011). Emotional words impact the mind but not the body: Evidence from pupillary responses. Psychophysiology. doi: 10.1111/j.1469-8986.2011.01219.x 43, 50

Berkum, J., Hagoort, P., \& Brown, C. (1999). Semantic integration in sentences and discourse: Evidence from the N400. Journal of Cognitive Neuroscience, 11(6), 657-671. doi: 10.1162/089892999563724 46

Bever, T. G. (1970). The cognitive basis for linguistic structures. In J. R. Hayes (Ed.), Cognition and the development of language (pp. 279-362). New York: Wiley. 14, 47

Boxer, D., \& Cortés-Conde, F. (1997). From bonding to biting: Conversational joking and identity display. Journal of Pragmatics, 27(3), 275-294. doi: 10.1016/S0378 $-2166(96) 00031-8 \quad 9$

Bradley, M. M., Miccoli, L., Escrig, M. A., \& Lang, P. J. (2008). The pupil as a measure of emotional arousal and autonomic activation. Psychophysiology, 45 (4), 602-607. doi: 10.1111/j.1469-8986.2008.00654.x 50, 111

Burkhardt, P. (2007). The P600 reflects cost of new information in discourse memory. NeuroReport, 18(17), 1851-1854. doi: 10.1097/WNR.0b013e3282f1a999 47

Calvo, M. G. (2000). The time course of predictive inferences depends on contextual 
constraints. Language and Cognitive Processes, 15(3), 293-319. doi: 10.1080/ $016909600386066 \quad 99,100$

Calvo, M. G., \& Castillo, M. D. (1996). Predictive inferences occur on-line, but with delay: Convergence of naming and reading times. Discourse Processes, 22(1), 57-78. doi: 10.1080/01638539609544966 90, 99

Carreiras, M. (1996). The use of stereotypical gender information in constructing a mental model: Evidence from English and Spanish. The Quarterly Journal of Experimental Psychology: Section A, 49(3), 639-663. doi: 10.1080/713755647 8, 46

Chan, Y.-C., Chou, T.-L., Chen, H.-C., \& Liang, K.-C. (2012). Segregating the comprehension and elaboration processing of verbal jokes: An fMRI study. NeuroImage, 61(4), 899-906. doi: 10.1016/j.neuroimage.2012.03.052 68

Chater, N., \& Manning, C. D. (2006). Probabilistic models of language processing and acquisition. Trends in Cognitive Sciences, 10(7), 335-344. doi: 10.1016/ j.tics.2006.05.006 19

Chwilla, D. J., Brown, C. M., \& Hagoort, P. (1995). The N400 as a function of the level of processing. Psychophysiology, 32(3), 274-285. doi: 10.1111/j.1469-8986 $.1995 . t b 02956 . x \quad 40$

Clore, G. L., \& Huntsinger, J. R. (2007). How emotions inform judgment and regulate thought. Trends in Cognitive Sciences, 11(9), 393-399. doi: 10.1016/j.tics.2007 $.08 .005 \quad 4$

Coulson, S. (2001). Semantic leaps: Frame-shifting and conceptual blending in meaning construction. Cambridge, UK: Cambridge University Press. 6, 30, 84, 86, 103, 107,134

Coulson, S., \& Kutas, M. (1998). Frame-shifting and sentential integration (Cognitive Science Technical Report (Tech. Rep.). La Jolla, CA: UCSD. Retrieved from 
http://citeseerx.ist.psu.edu/viewdoc/summary?doi=10.1.1.46.8181 30, $39,46,51,84,86,103,134$

Coulson, S., \& Kutas, M. (2001). Getting it: Human event-related brain response to jokes in good and poor comprehenders. Neuroscience Letters, 316(2), 71-74. doi: 10.1016/S0304-3940(01)02387-4 39, 40, 42, 47, 48, 65, 67, 69, 80, 86, 101, 103, 110,134

Coulson, S., \& Lovett, C. (2004). Handedness, hemispheric asymmetries, and joke comprehension. Cognitive Brain Research, 19(3), 275-288. doi: http://dx.doi.org/ 10.1016/j.cogbrainres.2003.11.015 40, 42, 47, 48, 67, 80, 81, 86, 101, 103, 110, 134

Coulson, S., \& Oakley, T. (2005). Blending and coded meaning: Literal and figurative meaning in cognitive semantics. Journal of Pragmatics, 37(10), 1510-1536. doi: 10.1016/j.pragma.2004.09.010 30

Coulson, S., Urbach, T. P., \& Kutas, M. (2006). Looking back: Joke comprehension and the space structuring model. Humor, 19(3), 229-250. doi: 10.1515/HUMOR $.2006 .01330,39,46,86$

Coulson, S., \& Williams, R. F. (2005). Hemispheric asymmetries and joke comprehension. Neuropsychologia, 43(1), 128-141. doi: 10.1016/j.neuropsychologia.2004.03 $.01547,48,86,110$

Cuthbert, B. N., Schupp, H. T., Bradley, M. M., Birbaumer, N., \& Lang, P. J. (2000). Brain potentials in affective picture processing: Covariation with autonomic arousal and affective report. Biological Psychology, 52(2), 95-111. doi: http://dx.doi.org/10.1016/S0301-0511(99)00044-7 48

Dascal, M. (1985). Language use in jokes and dreams: Sociopragmatics vs. psychopragmatics. Language and Communication, 5(2), 95-106. doi: http://dx.doi.org/ 10.1016/0271-5309(85)90002-3 13 
De Finetti, B. (1970). Logical foundations and measurement of subjective probability. Acta Psychologica, 34, 129-145. doi: 10.1016/0001-6918(70)90012-0 20

De Palma, P., \& Weiner, E. J. (1992). Riddles: Accessibility and knowledge representation. In Proceedings of the 14th conference on computational linguistics-volume 4 (pp. 1121-1125). doi: 10.3115/992424.992441 17, 28, 86, 88

Derks, P., Gillikin, L., Bartolome-Rull, D., \& Bogart, E. (1997). Laughter and electroencephalographic activity. Humor, 10(3), 285-300. doi: 10.1515/humr.1997.10.3.285 $47,65,110$

Dreisbach, G., \& Goschke, T. (2004). How positive affect modulates cognitive control: Reduced perseveration at the cost of increased distractibility. Journal of Experimental Psychology: Learning, Memory, and Cognition, 30(2), 343-353. doi: 10.1037/0278-7393.30.2.343 42

Du, X., Qin, Y., Tu, S., Yin, H., Wang, T., Yu, C., \& Qiu, J. (2013). Differentiation of stages in joke comprehension: Evidence from an ERP study. International Journal of Psychology, 48(2), 149-157. doi: 10.1080/00207594.2012.665162 49, 67, 81, 114

Dwivedi, V. D., Phillips, N. A., Einagel, S., \& Baum, S. R. (2010). The neural underpinnings of semantic ambiguity and anaphora. Brain Research, 1311, 93-109. doi: 10.1016/j.brainres.2009.09.102 79

Dynel, M. (2009). Humorous garden-paths: A pragmatic-cognitive study. Cambridge: Cambridge Scholars Publishing. 10, 14, 15, 16, 17, 22, 29, 35, 87, 92, 134

Dynel, M. (2012). Garden-paths, red lights and crossroads: On finding our way to understanding the cognitive mechanisms underlying jokes. Israeli Journal of Humor Research: An International Journal, 1, 6-28. Retrieved from http://s3.amazonaws.com/academia.edu.documents/ 30866899/1-_Garden_paths__red_lights_and_crossroads__Marta_Dynel 
-_-_D7\%A1\%D7\%95\%D7\%A4\%D7\%99_19_\%D7\%99\%D7\%95\%D7\%A0\%D7\%99_2012.pdf

?AWSAccessKeyId=AKIAJ56TQJRTWSMTNPEA\&Expires=1388773946\&Signature= vFJBYsgD6QbRXx7uYBLB4gxN35k\%3D\&response-content-disposition=inline $10,29,34$

Fauconnier, G. (1994). Mental spaces. Cambridge: Cambridge University Press. 6 Fauconnier, G., \& Turner, M. (2008). The way we think: Conceptual blending and the mind's hidden complexities. New York: Basic Books. 6

Ferreira, F., Christianson, K., \& Hollingworth, A. (2001). Misinterpretations of gardenpath sentences: Implications for models of sentence processing and reanalysis. Journal of Psycholinguistic Research, 30(1), 3-20. doi: 10.1023/A:1005290706460 45

Forabosco, G. (1992). Cognitive aspects of the humor process: The concept of incongruity. Humor-International Journal of Humor Research, 5(1-2), 45-68. doi: http://dx.doi.org/10.1515/humr.1992.5.1-2.45 5, 33, 36, 79

Frazier, L., \& Clifton Jr, C. (1997). Construal: Overview, motivation, and some new evidence. Journal of Psycholinguistic Research, 26(3), 277-295. doi: 10.1023/A: 102502452413323

Frazier, L., \& Fodor, J. D. (1978). The sausage machine: A new two-stage parsing model. Cognition, 6(4), 291-325. doi: 10.1016/0010-0277(78)90002-1 23

Fredrickson, B. (1998). What good are positive emotions?. Review of General Psychology, 2(3), 300-319. doi: http://psycnet.apa.org/doi/10.1037/1089-2680.2.3.300 3,111

Fredrickson, B., \& Branigan, C. (2005). Positive emotions broaden the scope of attention and thought-action repertoires. Cognition \& Emotion, 19(3), 313-332. doi: 10 $.1080 / 02699930441000238 \quad 4,111$

Freud, S. (1905). Der Witz und seine Beziehung zum Unbewussten. F. Deuticke. 108 
Gervais, M., \& Wilson, D. (2005). The evolution and functions of laughter and humor: A synthetic approach. The Quarterly Review of Biology, 80(4), 395-430. doi: $10.1086 / 4982812,3,4,108,111$

Gierych, E., Milner, R., \& Michalski, A. (2005). ERP responses to smile-provoking pictures. Journal of Psychophysiology, 19(2), 77-90. doi: 10.1027/0269-8803.19.2 $.77 \quad 48$

Giora, R. (1991). On the cognitive aspects of the joke. Journal of Pragmatics, 16(5), 465-485. doi: 10.1016/0378-2166(91)90137-M 5, 79

Giora, R. (1997). Understanding figurative and literal language: The graded salience hypothesis. Cognitive Linguistics, 8, 183-206. doi: http://dx.doi.org/10.1515/ $\operatorname{cog} 1.1997 .8 .3 .183 \quad 21,30$

Giora, R. (2003). On our mind: Salience, context, and figurative language. Oxford University Press New York. 17, 21, 30, 38, 86, 87

Giora, R., Fein, O., Laadan, D., Wolfson, J., Zeituny, M., Kidron, R., ... Shaham, R. (2007). Expecting irony: Context versus salience-based effects. Metaphor and Symbol, 22(2), 119-146. doi: 10.1080/10926480701235346 38

Goatly, A. (2012). Meaning and humour. Cambridge University Press. 87

Godkewitsch, M. (1976). Physiological and verbal indices of arousal in rated humour. In A. Chapman \& H. Foot (Eds.), Humor and laughter: Theory research and applications (pp. 117-138). London: John Wiley \& Sons. 111

Graesser, A. C., Millis, K. K., \& Zwaan, R. A. (1997). Discourse comprehension. Annual review of psychology, 48(1), 163-189. doi: 10.1146/annurev.psych.48.1.1639

Grice, H. P. (1975). Logic and conversation. In P. Cole \& J. Morgan (Eds.), Syntax and semantics (Vol. 3, pp. 41-58). New York: Academic Press. 9

Gruner, C. R. (2000). The game of humor: A comprehensive theory of why we laugh. Transaction Books. 108 
Hockett, C. F. (1973). Jokes. In M. Smith (Ed.), Studies in linguistics in honor of George L. Trager (pp. 153-178). The Hague: Mouton. 14

Hurley, M., Dennett, D., \& Adams, R. (2011). Inside jokes: Using humor to reverseengineer the mind. Cambridge, MA: MIT Press. 4, 6, 18, 23, 24, 32, 36, 107, 111, 134

Ille, N., Berg, P., \& Scherg, M. (2002). Artifact correction of the ongoing EEG using spatial filters based on artifact and brain signal topographies. Journal of Clinical Neurophysiology, 19(2), 113-124. Retrieved from http://www.ncbi.nlm.nih.gov/ pubmed/11997722?dopt=Abstract 59

Johnson-Laird, P. (1983). Mental models. Cambridge: Cambridge University Press. 45 Jurafsky, D. (2003). Probabilistic modeling in psycholinguistics: Linguistic comprehension and production. In Probabilistic linguistics (pp. 39-96). Cambridge, MA: MIT Press. 19, 23

Just, M., \& Carpenter, P. (1980). A theory of reading: From eye fixations to comprehension. Psychological Review, 87, 329-354. Retrieved from http://works.bepress.com/cgi/viewcontent.cgi?article=1105\&context= marcel_just_cmu\&sei-redir=1\&referer=http $\% 3 \mathrm{~A} \% 2 \mathrm{~F} \% 2 \mathrm{Fscholar}$.google .de $\% 2$ Fscholar $\% 3 F h 1 \% 3 D d e \% 26 q \% 3 D \% 2 B$ theory $\% 2 B$ of $\% 2$ Breading $\% 253 \mathrm{~A} \% 2 \mathrm{BFrom} \%$ 2Beye $\% 2 \mathrm{Bf}$ ixations $\% 2 \mathrm{Bto} \% 2 \mathrm{Bcomprehension} \% 26 \mathrm{btnG} \% 3 \mathrm{D} \% 26 \mathrm{lr} \% 3 \mathrm{D \# \text {search= }}$ $\% 22$ theory $\% 20$ reading $\% 3 \mathrm{~A} \% 2$ From $\% 20$ eye $\% 20$ fixations $\% 20$ comprehension $\% 22$ 90, 101

Just, M., Carpenter, P., \& Woolley, J. (1982). Paradigms and processes in reading comprehension. Journal of Experimental Psychology: General, 111(2), 228-238. doi: 10.1037/0096-3445.111.2.228 56, 90, 93, 99, 101

Kaan, E., \& Swaab, T. (2003). Repair, revision, and complexity in syntactic analysis: An electrophysiological differentiation. Journal of Cognitive Neuroscience, 15(1), 
98-110. doi: 10.1162/089892903321107855 47, 81

Kahneman, D., \& Beatty, J. (1966). Pupil diameter and load on memory. Science, 154(3756), 1583-1585. doi: 10.1126/science.154.3756.1583 50

Kintsch, W. (1988). The role of knowledge in discourse comprehension: A constructionintegration model. Psychological Review, 95(2), 163-182. doi: 10.1037/0033-295X $.95 .2 .163 \quad 9,45$

Kintsch, W. (1998). Comprehension: A paradigm for cognition. Cambridge: Cambridge University Press. 1, 45

Koestler, A. (1964). The act of creation. London: Hutchinson. 5, 7

Korb, S., Grandjean, D., Samson, A. C., Delplanque, S., \& Scherer, K. R. (2012). Stop laughing! Humor perception with and without expressive suppression. Social Neuroscience, 7(5), 510-524. doi: 10.1080/17470919.2012.667573 48

Kotthoff, H. (2006). Pragmatics of performance and the analysis of conversational humor. Humor-International Journal of Humor Research, 19(3), 271-304. doi: 10.1515/HUMOR.2006.015 9

Kotthoff, H. (2009). Joint construction of humorous fictions in conversation. an unnamed narrative activity in a playful keying. Journal of Literary Theory, 3(2), 195-217. doi: 10.1515/JLT.2009.012 9

Kreibig, S. D. (2010). Autonomic nervous system activity in emotion: A review. Biological Psychology, 84(3), 394-421. doi: 10.1016/j.biopsycho.2010.03.010 111 Kuhn, T. (1996). The structure of scientific revolutions. Chicago: University of Chicago Press. 32

Kutas, M., \& Federmeier, K. (2011). Thirty years and counting: Finding meaning in the N400 component of the event-related brain potential (ERP). Annual Review of Psychology, 62, 621-647. doi: 10.1146/annurev.psych.093008.131123 40, 46, 47 
Kutas, M., \& Hillyard, S. (1980). Reading senseless sentences: Brain potentials reflect semantic incongruity. Science, 207(4427), 203-205. doi: 10.1126/science.7350657 46

Kutas, M., \& Hillyard, S. A. (1984). Brain potentials during reading reflect word expectancy and semantic association. Nature, 307(5947), 161-163. 46

Levinson, S. C. (2000). Presumptive meanings: The theory of generalized conversational implicature. Cambridge, MA: MIT Press. 17, 22

MacDonald, M., \& Seidenberg, M. (2006). Constraint satisfaction accounts of lexical and sentence comprehension. In M. Traxler \& M. Gernsbacher (Eds.), Handbook of psycholinguistics (pp. 581-611). London: Elsevier Inc. 22, 23

Marinkovic, K., Baldwin, S., Courtney, M., Witzel, T., Dale, A., \& Halgren, E. (2011). Right hemisphere has the last laugh: Neural dynamics of joke appreciation. Cognitive, Affective, $\&$ Behavioral Neuroscience, 11(1), 1-18. doi: 10.3758/s13415-010-0017-7 48, 80

Martin, R. (2007). The psychology of humor: An integrative approach. Burlington: Elsevier Inc. 1, 2, 5, 10, 111

Martín-Loeches, M., Schacht, A., Casado, P., Hohlfeld, A., Rahman, R. A., \& Sommer, W. (2009). Rules and heuristics during sentence comprehension: Evidence from a dual-task brain potential study. Journal of Cognitive Neuroscience, 21(7), 13651379. doi: $10.1162 /$ jocn.2009.21106 47

Mayerhofer, B. (2013). Perspective clashing as a humour mechanism. In M. Dynel (Ed.), Developments in linguistic humour theory (pp. 211-233). Amsterdam: John Benjamins Publishing. 7, 9

Mayerhofer, B., \& Schacht, A. (2013). Salience, accessibility, and humorous potential in the comprehension of garden path jokes. In M. Dynel (Ed.), Developments in linguistic humour theory (pp. 341-366). Amsterdam: John Benjamins Publishing. 
$12,45,79,84,87,88$

McGhee, P. (1979). Humor: Its origin and development. San Francisco: WH Freeman. $5,33,79$

Meltzer, J. A., \& Braun, A. R. (2013). P600-like positivity and left anterior negativity responses are elicited by semantic reversibility in nonanomalous sentences. Journal of Neurolinguistics, 26(1), 129-148. 48

Minsky, M. (1975). A framework for representing knowledge. In O. Winston (Ed.), The psychology of computer vision. New York: McGraw-Hill. 31

Minsky, M. (1977). Frame-system theory. In P. Johnson-Laird \& P. C. Wason (Eds.), Thinking: Readings in cognitive science (pp. 355-376). Cambridge: Cambridge Univ. Press. 31

Minsky, M. (1984). Jokes and the logic of the cognitive unconscious. AI Memo No. 603 MIT. 7

Morris, R. K. (1994). Lexical and message-level sentence context effects on fixation times in reading. Journal of Experimental Psychology: Learning, Memory, and Cognition, 20(1), 92-103. doi: 10.1037/0278-7393.20.1.92 87, 99

Morris, R. K. (2006). Lexical processing and sentence context effects. In M. Traxler \& M. Gernsbacher (Eds.), Handbook of psycholinguistics (pp. 377-402). London: Elsevier Inc. 99, 102

Morris, S. B., \& DeShon, R. P. (2002). Combining effect size estimates in meta-analysis with repeated measures and independent-groups designs. Psychological Methods, 7(1), 105-125. doi: 10.1037/1082-989X.7.1.105 94

Mossbridge, J. A., Grabowecky, M., Paller, K. A., \& Suzuki, S. (2013). Neural activity tied to reading predicts individual differences in extended-text comprehension. Frontiers in Human Neuroscience, 7(655). doi: 10.3389\%2Ffnhum.2013.00655 110 
Münte, T. F., Schiltz, K., \& Kutas, M. (1998). When temporal terms belie conceptual order. Nature, 395(6697), 71-73. doi: 10.1038/25731 48

Nerhardt, G. (1977). Operationalization of incongruity in humour research: A critique and suggestions. In A. Chapman \& H. Foot (Eds.), It's a funny thing, humour (pp. 47-51). Oxford: Pergamon Press. 5, 33, 79

Norrick, N. R. (2001). On the conversational performance of narrative jokes: Toward an account of timing. Humor, 14(3), 255-274. doi: 10.1515/humr.2001.003 13

Norrick, N. R. (2009). A theory of humor in interaction. Journal of Literary Theory, 3(2), 261-283. doi: 10.1515/JLT.2009.015 9

Oaksford, M., \& Chater, N. (2007). Bayesian rationality: The probabilistic approach to human reasoning. Oxford: Oxford University Press. 19

Oldfield, R. (1971). The assessment and analysis of handedness: The Edinburgh inventory. Neuropsychologia, 9(1), 97-113. doi: 10.1016/0028-3932(71)90067-4 58,69, 75

Osterhout, L., \& Holcomb, P. (1992). Event-related brain potentials elicited by syntactic anomaly. Journal of Memory and Language, 31(6), 785-806. doi: 10.1016/0749 $-596 \mathrm{X}(92) 90039-\mathrm{Z} \quad 45,47$

Overeem, S., Lammers, G., \& Van Dijk, J. (1999). Weak with laughter. The Lancet, $354(9181), 838.111$

Partala, T., \& Surakka, V. (2003). Pupil size variation as an indication of affective processing. International Journal of Human-Computer Studies, 59(1-2), 185-198. doi: 10.1016/S1071-5819(03)00017-X 50

Paulos, J. A. (2008). Mathematics and humor: A study of the logic of humor. Chicago: University of Chicago Press. 134

Pexman, P. M., Ferretti, T. R., \& Katz, A. N. (2000). Discourse factors that influence online reading of metaphor and irony. Discourse Processes, 29(3), 201-222. doi: 
10.1207/S15326950dp2903_2 90, 91, 99

Pfeifer, N., \& Kleiter, G. (2010). The conditional in mental probability logic. In M. Oaksford \& N. Chater (Eds.), Cognition and conditionals: Probability and logic in human thought (pp. 153-173). Oxford: Oxford University Press. 20

Pivik, R., Broughton, R., Coppola, R., Davidson, R., Fox, N., \& Nuwer, M. (1993). Guidelines for the recording and quantitative analysis of electroencephalographic activity in research contexts. Psychophysiology, 30(6), 547-558. doi: 10.1111/ j.1469-8986.1993.tb02081.x 59

Ramachandran, V. (1998). The neurology and evolution of humor, laughter, and smiling: The false alarm theory. Medical Hypotheses, 51(4), 351-354. doi: 10.1016/S0306 $-9877(98) 90061-5 \quad 32$

Raskin, V. (1985). Semantic mechanisms of humor. Dordrecht: Reidel. 13

Raskin, V., \& Attardo, S. (1994). Non-literalness and non-bona-fide in language: An approach to formal and computational treatments of humor. Pragmatics $\&$ Cognition, 2(1), 31-69. doi: 10.1075/pc.2.1.02ras 9

Rayner, K., Kambe, G., \& Duffy, S. A. (2000). The effect of clause wrap-up on eye movements during reading. The Quarterly Journal of Experimental Psychology: Section A, 53(4), 1061-1080. doi: 10.1080/713755934 90

Rayner, K., Sereno, S. C., Morris, R. K., Schmauder, A. R., \& Clifton Jr, C. (1989). Eye movements and on-line language comprehension processes. Language and Cognitive Processes, 4(3-4), SI21-SI49. doi: 10.1080/01690968908406362 90

Ritchie, G. (2001). Current directions in computational humour. Artificial Intelligence Review, 16(2), 119-135. 134

Ritchie, G. (2004). The linguistic analysis of jokes. New York: Routledge. 10, 14, 15, $35,46,86,107$

Ritchie, G. (2006). Reinterpretation and viewpoints. Humor-International Journal of 
Humor Research, $19(3)$, 251-270. doi: 10.1515/HUMOR.2006.014 16

Ritchie, G. (2009). Variants of incongruity resolution. Journal of Literary Theory, 3(2), 313-332. doi: 10.1515/JLT.2009.017 33

Rosch, E. (1999). Principles of categorization. In E. Margolis \& S. Laurence (Eds.), Concepts: Core readings (pp. 189-206). Cambridge, MA: MIT Press. 87

Rothbart, M. K., \& Pien, D. (1977). Elephants and marshmallows: A theoretical synthesis of incongruity-resolution and arousal theories of humour. In A. Chapman \& C. Foot (Eds.), It's a funny thing, humour (pp. 37-40). Oxford: Pergamon Press. 34

Ruch, W. (1993). Exhilaration and humor. In M. Lewis \& J. Haviland (Eds.), Handbook of emotions (pp. 605-616). Guilford. 3, 111

Sanford, A. (1986). Cognition and cognitive psychology. New York: Basic Books, Inc. 8

Sanford, A. J., \& Garrod, S. C. (1998). The role of scenario mapping in text comprehension. Discourse Processes, 26 (2-3), 159-190. doi: 10.1080/01638539809545043 9

Schacht, A., Martín-Loeches, M., Casado, P., Rahman, R. A., Sel, A., \& Sommer, W. (2010). How is sentence processing affected by external semantic and syntactic information? Evidence from event-related potentials. PloS one, 5(3), e9742. doi: 10.1371/journal.pone.0009742 47

Schacht, A., \& Sommer, W. (2009a). Emotions in word and face processing: Early and late cortical responses. Brain and Cognition, 69(3), 538-550. doi: 10.1016/ j.bandc.2008.11.005 48

Schacht, A., \& Sommer, W. (2009b). Time course and task dependence of emotion effects in word processing. Cognitive, Affective, $\&$ Behavioral Neuroscience, 9(1), 28-43. doi: 10.3758/CABN.9.1.28 48

Schupp, H. T., Cuthbert, B. N., Bradley, M. M., Cacioppo, J. T., Ito, T., \& Lang, P. J. 
(2000). Affective picture processing: The late positive potential is modulated by motivational relevance. Psychophysiology, 37(2), 257-261. doi: 10.1111/1469 $-8986.3720257 \quad 48$

Seidenberg, M. S., \& MacDonald, M. C. (1999). A probabilistic constraints approach to language acquisition and processing. Cognitive Science, 23(4), 569-588. doi: 10.1016/S0364-0213(99)00016-6 22

Siebörger, F. T. (2006). Funktionelle Neuroanatomie des Textverstehens: Kohärenzbildung bei Witzen und anderen ungewöhnlichen Texten (Doctoral dissertation, MPI for Human Cognitive and Brain Sciences, University of Leipzig). Retrieved from http://pubman.mpdl.mpg.de/pubman/item/escidoc:722724: 2/component/escidoc:722723/sieboerger.pdf 84

Siebörger, F. T., Ferstl, E. C., \& von Cramon, D. Y. (2007). Making sense of nonsense: An fMRI study of task induced inference processes during discourse comprehension. Brain Research, 1166, 77-91. doi: 10.1016/j.brainres.2007.05.079 110

Simpson, J. A., Weiner, E. S., et al. (1989). The Oxford English dictionary (Vol. 2). Oxford: Clarendon Press. 2

Sturt, P. (2007). Semantic re-interpretation and garden path recovery. Cognition, 105(2), 477-488. doi: 10.1016/j.cognition.2006.10.009 46

Suls, J. (1972). A two-stage model for the appreciation of jokes and cartoons: An information-processing analysis. In G. Goldstein \& P. McGhee (Eds.), The psychology of humor: Theoretical perspectives and empirical issues (pp. 81-100). New York: Academic Press. 5, 10, 13, 33, 79, 107

Vaid, J., Hull, R., Heredia, R., Gerkens, D., \& Martinez, F. (2003). Getting a joke: The time course of meaning activation in verbal humor. Journal of Pragmatics, 35(9), 1431-1449. doi: 10.1016/S0378-2166(02)00184-4 38, 46, 86, 109

Van Berkum, J. J., Brown, C. M., Zwitserlood, P., Kooijman, V., \& Hagoort, P. (2005). 
Anticipating upcoming words in discourse: Evidence from ERPs and reading times. Journal of Experimental Psychology: Learning, Memory, and Cognition, 31 (3), 443-467. doi: http://psycnet.apa.org/doi/10.1037/0278-7393.31.3.443 99

Van Der Meer, E., Beyer, R., Horn, J., Foth, M., Bornemann, B., Ries, J., .. Wartenburger, I. (2010). Resource allocation and fluid intelligence: Insights from pupillometry. Psychophysiology, 47(1), 158-169. doi: 10.1111/j.1469-8986.2009.00884.x 50

Van Dijk, T. A., \& Kintsch, W. (1983). Strategies of discourse comprehension. New York: Academic Press. 88

Van Herten, M., Kolk, H. H., \& Chwilla, D. J. (2005). An ERP study of P600 effects elicited by semantic anomalies. Cognitive Brain Research, 22(2), 241-255. doi: 10.1016/j.cogbrainres.2004.09.002 42, 47, 83, 105

Võ, M. L.-H., Jacobs, A. M., Kuchinke, L., Hofmann, M., Conrad, M., Schacht, A., \& Hutzler, F. (2008). The coupling of emotion and cognition in the eye: Introducing the pupil old/new effect. Psychophysiology, 45(1), 130-140. doi: 10.1111/j.1469 $-8986.2007 .00606 . x \quad 50$

Vrticka, P., Black, J. M., \& Reiss, A. L. (2013). The neural basis of humour processing. Nature Reviews Neuroscience, 14(12), 860-868. doi: 10.1038/nrn3566 3

Werning, M., Fleischhauer, J., \& Beseoglu, H. (2006). The cognitive accessibility of synaesthetic metaphors. In Proceedings of the twenty-eighth annual conference of the cognitive science society (pp. 2365-2370). 87

Wicha, N. Y., Moreno, E. M., \& Kutas, M. (2004). Anticipating words and their gender: An event-related brain potential study of semantic integration, gender expectancy, and gender agreement in spanish sentence reading. Journal of Cognitive Neuroscience, 16(7), 1272-1288. doi: 10.1162/0898929041920487 47

Wyer, R. S., \& Collins, J. E. (1992). A theory of humor elicitation. Psychological 
Review, 99(4), 663-688. doi: 10.1037/0033-295X.99.4.66311, 68, 86, 88, 114

Yamaguchi, H. (1988). How to pull strings with words: Deceptive violations in the garden-path joke. Journal of Pragmatics, 12(3), 323-337. doi: 10.1016/0378 $-2166(88) 90036-7 \quad 14$

Zwaan, R., \& Radvansky, G. A. (1998). Situation models in language comprehension and memory. Psychological Bulletin, 123(2), 162-185. doi: 10.1037/0033-2909 $.123 .2 .162 \quad 9,87$

Zwaan, R., \& Rapp, D. (2006). Discourse comprehension. In M. Traxler \& M. Gernsbacher (Eds.), Handbook of psycholinguistics (pp. 725-764). London: Elsevier Inc. 9 


\section{Appendix A: Items from the Rating Study in Experiment 1}

(1) Der Text war mir zumindest sinngemäß bekannt. The text was familiar, even though not necessarily literally. (Familiarity)

(2) Ich habe den Text verstanden I did understand the text. (Comprehension)

(3) Der Text hat mich zum Lachen/Schmunzeln gebracht. The text made me laugh/smile. (Humor Behavioral)

(4) Der Text hat mich erheitert. The text amused me. (Humor Emotional)

(5) Der Text ist witzig. The text is funny. (Humor Cognitive)

(6) Der Text hat mich in die Irre geleitet. The text tricked me into the wrong way. (Predictability Behavioral)

(7) Das Ende des Textes hat mich überrascht. The ending of the text did surprise me. (Predictability Emotional)

(8) Das Ende des Textes ist vorhersehbar. The ending of the text is predictable. (Predictability Cognitive)

(9) Der Text ist verständlich. The text is understandable. (Comprehensibility Behavioral)

(10) Der Text hat mich verwirrt. The text confused me. (Comprehensibility Emotional)

(12) Der Text ist Unsinn. The text is nonsense. (Comprehensibility Cognitive) 


\section{Appendix B: Stimulus Material}

Stimulus material was taken and adapted from internet collections (retrieved from http://www.ahajokes.com/, http://www.jokedatabase.org, www.dein-witz.de, witze -datenbank.de, www.witze-ohne-ende.de, http://www.brainyquote.com/quotes/ authors/s/steven_wright.html, in August, 2011), from various books and previous articles on verbal humor (Attardo \& Raskin, 1991; Coulson \& Kutas, 1998, 2001; Coulson, 2001; Coulson \& Lovett, 2004; Dynel, 2009; Hurley et al., 2011; Paulos, 2008; Ritchie, 2001), from personal memory, and from friends.

- Mami, ich bin jetzt vierzehn Jahre alt, darf ich endlich einen BH und Make-up tragen? Nein, und iss deine Suppe auf, mein ... Junge/Mädchen/Onkel!

(Primes in Experiment 5: Radiergummi/Barbie/Spielzeugauto)

- Der Polizist hält eine Autofahrerin an und ermahnt sie: Sie können doch nicht mit 80 durch das Ortsgebiet fahren. Darauf erwidert die Dame: 80! Ich muss wohl mal wieder ... liften/bremsen/husten! (Arbeit/Tachometer/Faltern)

- Ein Patient klingelt beim Haus seines Arztes. Die Frau des Arztes öffnet die Tür. Mit kranker Stimme flüstert der Patient: Ist der Arzt da? Die Frau flüstert zurück: Nein, kommen Sie ... herein/morgen/daneben! (Schuh/Stetoskop/Bettlaken)

- Eine Frau fragt die Verkäuferin im Kleiderladen. Dürfte ich das Kleid im Schaufenster anprobieren? Die Verkäuferin antwortet: Ja, aber besser wäre die .. . Kabine/Bluse/Soße. (Magnet/Umkleide/Öffentlichkeit) 
- Ein Freund zu seinem Freund, der gerade aus einer Wellness-Therme kommt: Hast du ein Bad genommen? Darauf der andere: Nein, nur ...

Handtücher/Massagen/Kaninchen.

(Salat/Wasser/Zubehör)

- Ich lasse meinen Steuerberater meine Buchhaltung durchführen. Das spart Zeit. Letztes Jahr ersparte ich mir dadurch zehn ...

Jahre/Tage/Schuhe.

(Bleistift/Freizeit/Handschellen)

- Ein Mann zum Barkeeper: Können Sie mir etwas empfehlen, das kalt ist und voller Rum? Der Barkeeper antwortet: Ja, ich empfehle Ihnen meine ...

Frau/Drinks/Musik.

(Verschwendung/Vorschlag/Ehekrise)

- Eine neue Studie untersuchte die Schlafgewohnheiten von Studenten. Der durchschnittliche Student steht um halb 8 Uhr in der Früh auf. Dann geht er ... schlafen/lernen/werfen.

(Versuch/Morgensonne/Party)

- Eine neue Studie besagt, dass in Deutschland pro Jahr 720 Millionen Euro für Glücksspiel ausgegeben werden. Gut fünfzig Prozent davon entfallen auf ...

Heiraten/Pokern/Forschen.

(Stuhl/Brautkleid/Roulette)

- Meine Freundin hat soviel Negatives über Rauchen gelesen. Deshalb hat sie beschlossen, aufzuhören, mit dem ...

Lesen/Rauchen/Reden.

(Reue/Gesundheit/Ignoranz)

- Ein Bauer erzählt einem anderen Bauern: Gestern hab ich den Hof und die Ernte gegen Feuer und Hagel versichert. Darauf der andere Bauer: Gegen Feuer, das finde ich gut. Aber Hagel kann man nicht ...

erzeugen/versichern/bedienen.

(Verstand/Unwetter/Betrug)

- Ich wurde aus dem Kino geworfen, weil ich mein eigenes Essen mitbrachte. Aber die Preise für die Snacks im Kino sind so unverschämt teuer. Außerdem hatte ich Lust auf ... 
Gegrilltes/Sandwiches/Badminton.

(Kalender/Popcorn/Würstchen)

- Immer wenn ich sie lachen sehe, Fräulein Susi, möchte ich zu ihnen sagen: Bitte kommen Sie doch mal zu mir. Na, na, Sie sind mir vielleicht ein Casanova! Nein, ich bin ein ...

Zahnarzt/Liebhaber/Schriftsteller.

(Aufruhr/Flirt/Hygiene)

- Der Klassenlehrer zur stolzen Mutter: Ihr Sohn ist außerordentlich kreativ und voller origineller Ideen. Vor allem im ...

Rechtschreiben/Musizieren/Produzieren.

(Idee/Intelligenz/Nachhilfe)

- Die Frau zieht ihren Mann in das Schlafzimmer und sagt zu ihm: So, bitte zieh meine Bluse, meinen $\mathrm{BH}$ und meine Unterhose aus. Ich will nicht, dass du meine Sachen ...

anprobierst/zerreißt/verkaufst.

(Vergesslichkeit/Leidenschaft/Travestie)

- Ein Freund beschwert sich bei einem anderen: Meine Freundin meint, ich bin viel zu neugierig. Zumindest schreibt sie das in ihrem ...

Tagebuch/Reisebericht/Scheckbuch .

(Vorsicht/Verleumdung/Schnüffelei)

- Eine Frau sagt zu ihrer Freundin: Mein Mann ist ein Engel. Die andere antwortet: Da hast du aber mehr Glück als ich, weil meiner noch ...

lebt/trinkt/schläft.

(Intelligenz/Charme/Himmel)

- Der Arzt sagt zum Patienten: Ich kann die Ursache ihrer Beschwerden nicht entdecken. Ehrlich gesagt, glaube ich, das kommt vom Trinken. Der Patient antwortet: Gut, ich komme in einer Woche wieder. Vielleicht sind Sie dann ... nüchtern/schlauer/verwaist.

(Sieg/Diagnose/Inkompetenz)

- Sag mal Susi. Warum fütterst du deinen Kater mit Sonnenblumenkernen? Susi antwortet: Sonnenblumenkerne sind das Lieblingsfutter von meinem ... Vogel/Kater/Affen. 
(Pflanze/Haustier/Raubtier)

- Eine Kuh sagt zur anderen: Fürchtest du dich eigentlich vor dem Rinderwahn? Die andere Kuh antwortet: Warum sollte ich? Ich bin doch ein ...

Flugzeug/Hausrind/Enkelkind!

(Ungenau/gesund/verrückt)

- Der Schwimmer fragt den Lifeguard: Und sie sind auch sicher, dass hier keine Haigefahr herrscht? Der Lifeguard beruhigt den Schwimmer: Ja, ganz sicher. Die vertragen sich nicht mit den ...

Krokodilen/Algenarten/Ergebnissen.

(Verärgert/gefährlich/harmlos)

- Der Ehemann zu seiner Frau: Schatz. Ich habe einen tollen Job gefunden. Die Frau findet das wunderbar. Darauf sagt der Mann: Toll, morgen ist der erste Tag für ...

dich/mich/ihn.

(Niederlage/Anstellung/Bevormundung)

- Was ist denn mit dir los? fragt der Wirt den unglücklichen Stammgast. Ach, meine Frau ist mit meinem besten Freund durchgebrannt. Alles ist so sinnlos ohne ...

Freund/Frau/Ball.

(Radio/Liebe/Fußball)

- Ich würde gerne so wie mein Großvater sanft und ruhig im Schlaf sterben. Nicht angsterfüllt und schreiend wie seine ...

Fahrgäste/Kameraden/Vertreter.

(Notizbuch/Bett/Straßenverkehr)

- Ein Blinder kommt mit seinem Hund an der Leine in eine Bar. Plötzlich schwingt er den Hund über seinem Kopf mit der Leine im Kreis. Der Barkeeper schreit ihn an: Was machen Sie da? Der Blinde antwortet: Ach, einfach nur ...

herumschauen/herumschwingen/herumschwimmen.

(Heizung/Quälerei/Sehhilfe)

- Die alte Hebamme kommt völlig aufgelöst zum Pfarrer: Mein Mann ist gestern verstorben. Der Pfarrer versucht sie zu trösten: Das ist ja furchtbar. Hatte er noch einen letzten Wunsch? Sie antwortet: Ja, seine letzten Worte waren: Bitte 
nicht ...

schießen/weinen/hören!

(Zahnpasta/Witwe/Pistole)

- Der Sohn eines Rechtsanwalts fragt seinen Vater: Sagt ein Rechtsanwalt jemals die Wahrheit? Der Vater antwortet: Ja, ein Rechtsanwalt macht alles, was hilft, um einen Fall zu ...

gewinnen/klären/teilen.

(einfühlsam/ehrlich/ehrgeizig)

- Ein Rechtsanwalt verteidigt den Angeklagten, der seine beiden Eltern ermordete: Ich plädiere auf mildernde Umstände für meinen Klienten, bedenken Sie, er ist ein

Waisenkind/Geisteskranker/Kartenleger.

(Hochzeit/Adoption/Blut)

- Ein Arzt hatte Sex mit einem seiner Patienten. Er beichtet es einem Freund und rechtfertigt sich bei ihm: Ich bin sicher, dass machen viele andere Ärzte auch. Der Freund antwortet: Ja, aber ich denke, von denen bist du der einzige ...

Tierarzt/Frauenarzt/Malermeister.

(Frosch/Mensch/Pferd)

- Sie hat ihr gutes Aussehen von ihrer Mutter. Die Mutter ist ...

Schönheitschirurgin/Unterwäschenmodel/Drogenkommissarin.

(Kiste/Gene/Skalpell)

- Ich leide nicht mehr unter Geisteskrankheit. Jeden einzelnen Augenblick meines Lebens kann ich die Krankheit ...

genießen/kontrollieren/vorbereiten.

(intelligent/gesund/verrückt)

- Ich habe zwei Warnschüsse abgefeuert. Beide in den ...

Kopf/Himmel/Ofen.

(Löffel/Angst/Blut)

- Letztens habe ich mitansehen müssen, wie sechs Männer meinen Chef verprügelten. Ich fühlte mich schlecht, weil ich nicht geholfen habe. Aber sechs Männer sind echt ...

genug/zuviel/anders. 
(Stolz/Gewissen/Schadenfreude)

- Ich habe noch immer den Körper einer 18-Jährigen. In meinem ... Keller/Alter/Bild. (Auto/Diät/Kerker)

- Dreißig Jahre lang waren meine Frau und ich glücklich und zufrieden. Dann haben wir uns ... getroffen/getrennt/beliefert. (Aufregung/Eheglück/Ehefrust)

- Über vierzig Jahre lang habe ich nun nur eine Frau geliebt. Ich hoffe, dass meine Frau das nie... erfährt/vergisst/mietet.

(Wertschätzung/Zufriedenheit/Ehebetrug)

- Ich bin so stolz. Viermal die Woche gehe ich ins Fitness-Center. Bald mache ich dort auch einen ... Work-Out/Yoga-Kurs/Surf-Kurs. (Pullover/Liegestütze/Bierbauch)

- Ich hasse es, wenn Leute Freddie Mercury mit Gott vergleichen. Ich meine, er ist gut. Aber doch nicht so gut wie der ...

Rockstar/Allmächtige/Zirkusdirektor.

(Seife/Mikrophon/Himmel)

- Die OP-Assistentin warnt den Chirurgen: Doktor, schneiden Sie nicht zu tief. Sie beschädigen sonst den ... OP-Tisch/Herzmuskel/Autolack. (Linie/Stirnrunzeln/Kratzer)

- In New York wird alle zwei Minuten eine Person von einem Auto überfahren. Das ist eine große gesundheitliche Gefahr für die ...

Person/Einwohner/Polizei.

(Einverständnis/Statistik/Pechvogel)

- Ich habe den Wien-Marathon in unter drei Stunden beendet. Ich wäre eigentlich noch schneller gewesen. Aber ich musste eine längere Pause machen, um zu ... tanken/trinken/tanzen. 
(Papier/Medaille/Schummler)

- Ich bin kürzlich auf einem Pferd geritten. Der hintere Fuß des Pferdes war so stark beeinträchtigt, dass ich es leider erschießen musste. Alle waren total darüber entsetzt, also alle anderen in dem ...

Kinderkarussel/Reitverein/Turnverein.

(Träumerei/Euthanasie/Verrücktheit)

- Um vier Uhr in der Früh ging heute der Autoalarm von meinem Nachbarn los. Ich bin so furchtbar erschrocken, dass ich sofort ...

weglief/aufwachte/aufaß.

(Türschild/Schlafzimmer/Brechstange)

- Glaub mir! Dir wehzutun, ist das Allerletzte, was ich vorhabe. Aber es ist ... eingeplant/unvermeidbar/unberührbar.

(introvertiert/lieb/gemein)

- Einmal pro Woche gehen meine Frau und ich in ein schickes Restaurant, danach schauen wir einen romantischen Film im Kino, und danach haben wir leidenschaftlichen Sex. Manchmal machen wir das auch ...

miteinander/mehrmals/minütlich. (Flucht/Kerzenschein/Affäre) 


\section{Appendix C: Data Base and Intercorrelations of Stimulus Variables}

The empirical data of this dissertation projects contains data from: ratings (see Experiment 1) $(N=68)$, Cloze-probability task $(N=65$, Joke, Coherent), self paced reading times (see Experiment 1$)(N=24$, all three conditions), self paced reading times $(N=45$, only jokes), 2 ERP studies $(N s=21,24$, N400, LLAN, LPs), Pupil dilation $(N=21)$

Variables of the data base that were included for the intercorrelations were:

Predictability Sum of the predictability rating of a joke in Experiment 1.

Comprehensibility Sum of the comprehensibility rating of a joke in Experiment 1.

Humor Potential Sum of the humor potential rating of a joke in Experiment 1.

LZ Reading time of the punch-line (final word) of a joke aggregated over participants in Experiment 1.

lz2pl Reading time of the punch-line (final word) of a joke aggregated over participants in Experiment 5.

lz2fs Average reading time of the follow-up sentence of a joke aggregated over participants in Experiment 5.

clozeJ The Cloze-probability of the joke ending of a joke.

clozeC The Cloze-probability of the coherent ending of a joke.

Pupil The pupil dilation between 800 and $2000 \mathrm{~ms}$ after the onset of the punch-line of a joke aggregated over participants in Experiment 2.

N400 The mean amplitude of the N400-ROI between 250 and $500 \mathrm{~ms}$ after the onset of the punch-line of a joke aggregated over participants in Experiment 2 and Experiment 4.

LLAN The mean amplitude of the LLAN-ROI between 500 and $700 \mathrm{~ms}$ after the onset of the punch-line of a joke aggregated over participants in Experiment 2 and Experiment 4. 
Table 5.1: The most highly correlated variables of the data base. Correlations are based on the Spearman-method.

\begin{tabular}{lcr}
\hline \hline First Variable & Second Variable & Correlation \\
\hline Comprehensibility & Predictability & .51 \\
Humor Potential & Comprehensibility & .5 \\
Humor Potential & LZ2fs & -.44 \\
clozeJ & N400 & .44 \\
Comprehensibility & LZ2pl & -.42 \\
LZ & LLAN & -.4 \\
Humor Potential & Pupil & .39 \\
clozeJ & LZ2pl & -.38 \\
Predictability & LZ2pl & -.37 \\
Predictability & LZ2fs & -.36 \\
Comprehensibility & LZ2fs & -.35 \\
Predictability & N400 & .35 \\
clozeC & clozeJ & .27 \\
Comprehensibility & clozeJ & .26 \\
N400 & LLAN & -.26 \\
\hline \hline
\end{tabular}

LP The mean amplitude of the fP600-ROI between 700 and $1000 \mathrm{~ms}$ after the onset of the punch-line of a joke aggregated over participants in Experiment 2 and Experiment 4. 


\section{Bastian Mayerhofer}

\section{Personal details}

$\begin{array}{llll}\text { Address: } & \text { Riemannstraße 1 } & \text { Date of Birth: } & 17 \text { November } 1983 \\ & 37083 \text { Göttingen } & \text { Nationality: } & \text { Austrian } \\ & \text { Germany } & \text { Marital Status: } & \text { Unmarried } \\ \text { Mobile: } & 0043 / 6642659673 & \text { E-mail: } & \text { Baasztian@gmail.com } \\ & 0176 / 78316824 & & \text { bmayerh@gwdg.de }\end{array}$

\section{Education}

Oct 2011 - date

Georg August Universtät Göttingen, Germany

$\mathrm{PhD}$ student in Experimental Psycholinguistics

Oct 2005 - Nov 2010

Paris Lodron Universität Salzburg, Austria

Diploma Psychology (M.Sc., 5 years and equivalent to a master's degree)

Feb 2006 - Jan 2010

Paris Lodron Universität Salzburg, Austria

Master's degree in Germanistik (M.A., Focus on German linguistics)

Sept 2007 - July 2008 Università degli Studi di Bologna, Italy

Erasmus Exchange "Psicologia Cognitiva Applicata" (Applied Cognitive Psychology)

Oct 2003 - Apr 2006

Paris Lodron Universität Salzburg, Austria

Bachelor's degree in Germanistik (B.A.)

Oct 2002 - July $2003 \quad$ Alma Mater Universität Wien, Austria

Theatre-, Film and Media Sciences (not accomplished)

\section{Work experience}

Feb 2011 - date

Nov 2008 - June 2010

Aug 2009 - Oct 2009

and Sept $2010-$ Dec 2010
Research assistant at CRC Textstrukturen, Excellence Initiative of the Georg August Universität Göttingen, in the Junior Research Group "Experimental Psycholinguistics" by Annekathrin Schacht, Göttingen Germany

Research assistant in the LogiCCC project of Gernot Kleiter, Salzburg, Austria

Research assistant in the group of Peter Juslin, Uppsala, Sweden

\section{Publications}

- Mayerhofer, B. \& Schacht, A. (in preparation). From incoherence to mirth: Neurocognitive processing of garden-path jokes.

- Mayerhofer, B., Schacht, A., \& Maier, K. (in revision). Manipulating salience and accessibility: Priming interpretations in garden-path jokes.

- Mayerhofer, B. \& Schacht, A. (2013). Salience, accessibility, and humorous potential in the comprehension of garden path jokes: A probabilistic approach. In M. Dynel (ed.), 
Developments in linguistic humour theory (pp. 341-366).

- Mayerhofer, B. (2013). Perspective clashing as a humour mechanism. In M. Dynel (Ed.), Developments in linguistic humour theory (pp. 211-233). Amsterdam: John Benjamins Publishing.

- Juslin, P., Mayerhofer, B., \& Lindskog M. (in revision). Are There Special Cognitive Algorithms for Integration of Probabilities?

- Fugard A., Pfeifer N., Mayerhofer B., \& Kleiter G. (2011). How people interpret conditionals: Shifts towards the conditional event. Journal of Experimental Psychology: Learning, Memory, and Cognition, 37(3), 635-648. doi: 10.1037/a0022329

- Fugard A., Pfeifer N., \& Mayerhofer B. (2011). Probabilistic theories of reasoning need pragmatics too: Modulating relevance in uncertain conditionals. Journal of Pragmatics, 43(7), 2034-2042. doi: 10.1016/j.pragma.2010.12.009

\section{Workshop/conference participations and presentations}

May 2013

March 2013

Nov 2012

Oct 2012

June 2012

May 2012

May 2012

July 2011

Feb 2009 and Feb 2010

Sept 2009

July 2009
Text in Perspective: Processing and Interpretation. Göttingen, Germany. Poster presentation: "Neuro-Cognitive Processes During the Comprehension of Garden-Path Jokes" Bastian Mayerhofer and Annekathrin Schacht.

LAFAL Linguistic Approaches to Funniness, Amusement and Laughter. Lodz, Poland. Presentation: "Salience vs. Accessibility. A Probabilistic Approach and Primed Interpretations in Garden Path Jokes." Bastian Mayerhofer, Katja Maier, and Annekathrin Schacht.

2. Studentische Tagung Sprachwissenschaft (StuTS 52), Berlin, Germany. Presentation: "This joke walks into an EEG lab. Belief Revision in Semantic-Pragmatic Garden-Path Jokes." Bastian Mayerhofer and Annekathrin Schacht.

Fall School Shifting Perspectives. Non-canonical Forms of Reported Discourse in Spoken and Sign Languages, Göttingen, Germany. Presentation: "Perspective Clashing as a Humour Mechanism." Bastian Mayerhofer.

International Society for Humor Studies Conference. Krakow, Poland. Presentation: "Belief Revision in Semantic-Pragmatic Garden Path Jokes." Bastian Mayerhofer and Annekathrin Schacht.

Cusanus Tagung der Fachschaft Neuphilologie, "Erst sprechen dann denken." Presentation: "'Belief revision' in semnatisch-pragmatischen Garden-Path Witzen". Bastian Mayerhofer and Annekathrin Schacht.

Research Colloquium at the Georg-Elias-Müller Institut, Göttingen. Presentation: "Belief Revision in Semantic-Pragmatic Garden Path Jokes." Bastian Mayerhofer and Annekathrin Schacht.

$11^{\text {th }}$ International Summer-School of Humour, Tartu, Estonia. Posterpresentation: "Belief Revision in Semantic Garden-Path Jokes: an ERP Study'. Bastian Mayerhofer and Annekathrin Schacht.

PROBNET: Salzburg, Austria

WUPES: Liblice, Czech Republic

London Reasoning Workshop 2009: London, UK. 


\section{Teaching activity}

2011/2012 and 2012/2013

$2011 / 2012$

$2012 / 2013$
"Humor im Text aus psycho-linguistischer Perspektive (humour in texts from a psycho-linguistic perspective)" at the Seminar für Deutsche Philologie (German studies department) at the Georg August Universität Göttingen, Germany (positively evaluated)

Supervision of the M.Sc. thesis by Katja Maier: "Primed down the garden path"

(Assistant) Supervision of the B.A. thesis by Janna Hinrichs 